\title{
ALL-FIBER PASSIVELY Q-SWITCHED \\ YTTERBIUM DOPED DOUBLE-CLAD FIBER LASERS: \\ EXPERIMENT AND MODELING
}

by

\author{
Yi Lu \\ A thesis presented to \\ Ryerson University \\ in partial fulfillment of the \\ requirements for the degree of \\ Master of Applied Science \\ in the Program of \\ Electrical and Computer Engineering
}

Toronto, Ontario, Canada, 2013

(C) Copyright by Yi Lu 2013 


\section{AUTHOR'S DECLARATION FOR ELECTRONIC SUBMISSION OF A THESIS}

I hereby declare that I am the sole author of this thesis. This is a true copy of the thesis, including any required final revisions, as accepted by my examiners.

I authorize Ryerson University to lend this thesis to other institutions or individuals for the purpose of scholarly research.

I further authorize Ryerson University to reproduce this thesis by photocopying or by other means, in total or in part, at the request of other institutions or individuals for the purpose of scholarly research.

I understand that my thesis may be made electronically available to the public. 


\title{
ALL-FIBER PASSIVELY Q-SWITCHED YTTERBIUM DOPED DOUBLE-CLAD FIBER LASERS: EXPERIMENT AND MODELING
}

\author{
Yi Lu \\ Master of Applied Science \\ Electrical and Computer Engineering \\ Ryerson University
}

2013

\begin{abstract}
All-fiber passively Q-switched lasers were demonstrated using ytterbium-doped double cladding fiber (YDF) as an active medium. The laser was pumped by three 25W, 975nm fiber coupled diodes and Q-switching was initiated when the amplified spontaneous emission generated in the core of the gain fiber bleached the saturable absorber (SA). A piece of samarium-doped fiber was used as SA in first configuration and pulses with $68 \mu \mathrm{J}$ pulse energy and 210ns pulse width were obtained. In second configuration, a piece of ytterbium-doped fiber with much smaller core size was used as SA to produce pulse energy of $86 \mu \mathrm{J}$. The last configuration incorporated a 9m-long YDF as gain fiber. The far end from pump was acting as SA in this case and pulses with $82 \mu \mathrm{J}$ pulse energy and 148ns pulse width were observed. The peak power was estimated at 554W. Traveling wave model was implemented to numerically simulate the output characteristics versus pump power.
\end{abstract}




\section{Acknowledgments}

It is a great pleasure for me to acknowledge many people who made this thesis possible. First and foremost I would like to express my sincere gratitude to my supervisor, Dr. Xijia Gu for his expert supervision and guidance, continuous support and patience during the course of this work. I am grateful for the opportunity given by Dr. Gu to be part of the Fiber Optics and Sensing Laboratory and study in an upcoming research area. For the past two years, his patience, motivation, enthusiasm and immense knowledge have contributed a lot to my personal growth. I could not have imagined having a better advisor for my M.A.Sc. study.

I also want to express my gratitude to Jiang Li and Angie R. Eldamak for the help in assembling laser modules. Jiang's expertise in fiber optics, Bragg gratings, and fusion splicer is invaluable in successful completion of this work. My sincere thanks also go to Prof. Lian Zhao and Prof. Kaamran Raahemifar from whose lectures I benefited greatly. I also want to thank all the colleagues and friends from Fiber Optics and Sensing Laboratory for all the interesting discussions and fruitful comments and for making our laboratory a pleasant work place.

Last but not least I want to express my deepest love to my parents and my wife for their constant encouragement and endless support. Without their support, I would never reach this far. 


\section{Table of Contents}

ACKNOWLEDGMENTS

CHAPTER 2 ALL-FIBER PASSIVELY Q-SWITCHED FIBER LASER WITH SM-DOPED FIBER

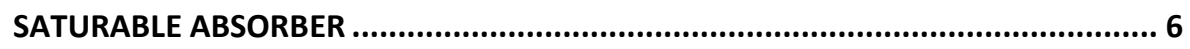

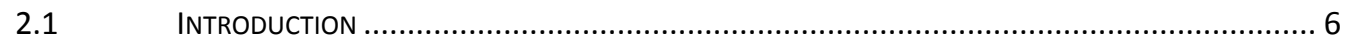

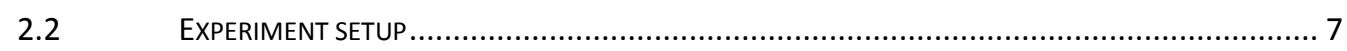

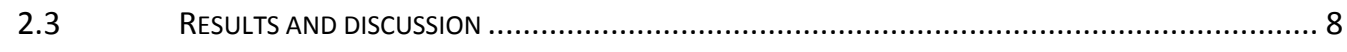

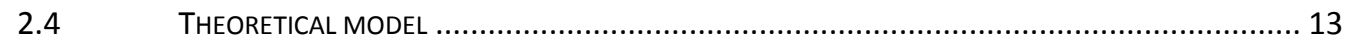

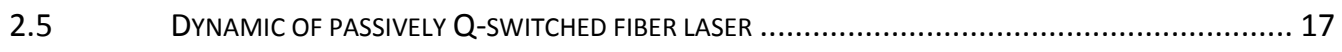

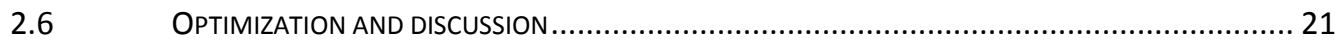

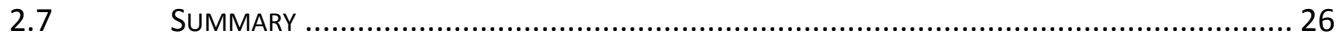

CHAPTER 3 ALL-FIBER PASSIVELY Q-SWITCHED FIBER LASER WITH YB-DOPED FIBER

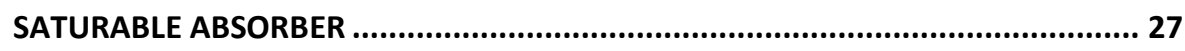

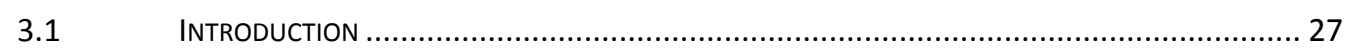

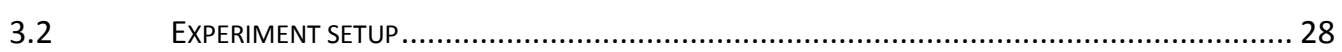

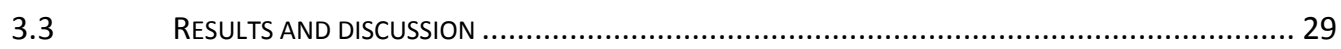

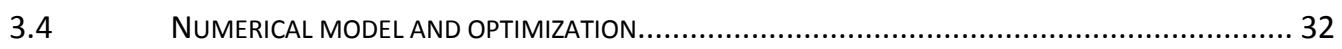

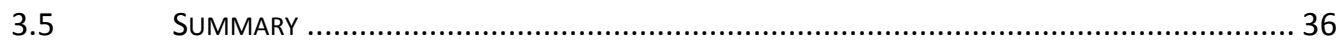

CHAPTER 4 ALL-FIBER PASSIVELY Q-SWITCHED FIBER LASER WITH EXCESSIVE LONG YBDOPED FIBER.................................................................................................... 38

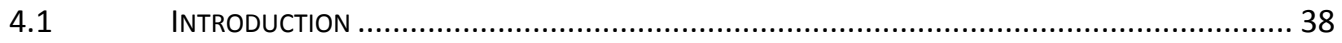




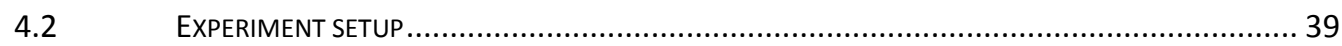

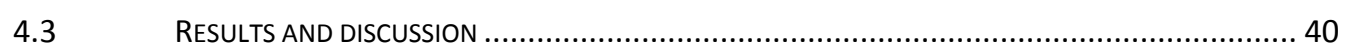

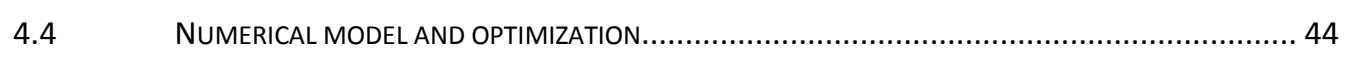

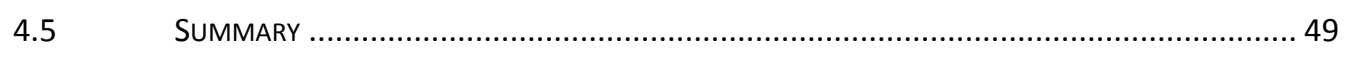

CHAPTER 5 CONCLUSION AND FUTURE WORK ......................................................... 51

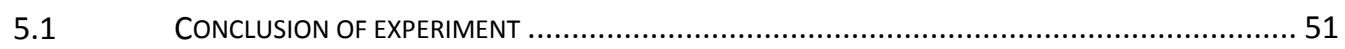

CONCLUSION OF THEORETICAL MODELING ............................................................ 52

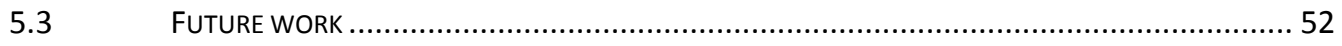

APPENDIX A CALCULATION OF DOPANT CONCENTRATION FOR ACTIVE FIBERS ..................... 54

APPENDIX B MATERIAL PROCESSING APPLICATION .................................................... 56

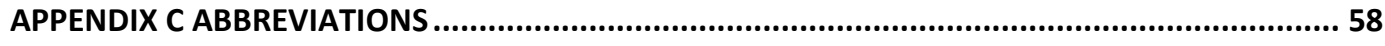

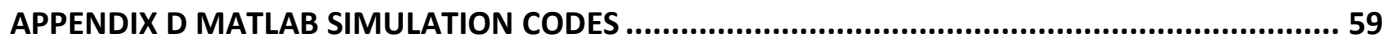

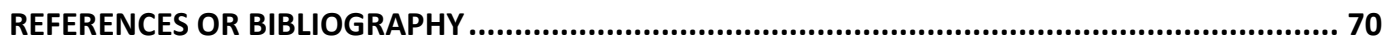




\section{List of Tables}

TABLE 2.1 SiMULATION PARAMETERS IN PASSIVELY Q-SWITCHED FIBER LASER WITH SM-DOPED SA. ............... 16

TABLE 3.1 SimUlation PARAMETERS IN PASSIVELY Q-SWITCHED FIBER LASER WITH YB-DOPED SA. ................. 34

TABLE 4.1 SIMULATION PARAMETERS IN PASSIVELY Q-SWITCHED FIBER LASER WITH EXCESSIVE LONG YB-DOPED

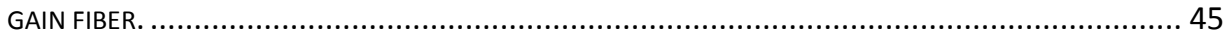




\section{List of Figures}

FIG. 2.1 CONFIGURATION DIAGRAM OF PASSIVELY Q-SWITCHED ALL-FIBER LASER INCORPORATING MODE FIELD MISMATCHING.

Fig. 2.2 (A) OSCILLOSCOPE TRACE OF THE Q-SWITCHING PULSES AT $100 \mathrm{KHZ}$ RATE; (B) A SINGLE PULSE WITH 68 MJ ENERGY AND 210 NS (FWHM) PULSE DURATION. (c) OSCILLOSCOPE TRACES OF THE QSWITCHING PULSES AT REPETITION RATE OF $10 \mathrm{kHZ}$, AND (D) AT $60 \mathrm{kHz}$. 9

FIG. 2.3 (A) A COMPARISON OF PULSED PUMP POWER (DOTS) AND OUTPUT LASER PULSES (SOLID) AT $100 \mathrm{KHZ}$; (B) A Q-SWITCHING PULSE TRAIN WITH RELAXATION OSCILLATION PULSES PUMPED AT 100 KHZ AND ABOVE 31\% DUTY-CYCLE THAN NORMAL OPERATION CONDITION.

Fig. 2.4 (A) EMISSION SPECTRUM OF THE LASER At 100 KHz; AND (B) AVERAGE LASER POWER VS. AVERAGE PUMP POWER OPERATED AT $100 \mathrm{KHZ}$.

FIG. 2.5 (A) PUMP DURATION VS. PUMP AMPLITUDE; AND (B) LASER PULSE WIDTH AND PULSE ENERGY VERSUS PUMP POWER AMPLITUDE. THE LASER IS OPERATED AT 100 KHZ WITH A 30 CM LONG OF SA. ........ 12

FIG. 2.6 TRAVELLING WAVE MODEL FOR THE PASSIVELY Q-SWITCHED FIBER LASER WITH SA FIBER. 13

Fig. $2.7 \quad$ A) THEORETICALly SIMULATED Q-SWITCHING PULSE TRAINS AT $100 \mathrm{kHz}$ B) OSCILLOSCOPE TRACE OF THE Q-SWITCHING LASER UNDER THE SAME OPERATION CONDITION.

FIG. 2.8 A SINGLE PULSE (SOLID) ALONG WITH THE CORRESPONDING PUMP POWER (LOOSELY DASHED), INVERSION IN GAIN FIBER (DASHED), AND INVERSION IN SA (DASH-DOTED) GENERATED WITH 30CM SA AND 70W PUMP AMPLITUDE. 18

FIG. 2.9 PULSE SHAPE, INVERSION IN GAIN FIBER, AND INVERSION IN SA GENERATED WITH DIFFERENT SA LENGTH AND PUMP AMPLITUDE. 19

FIG. 2.10 INVERSION DISTRIBUTION IN ACTIVE FIBER UNDER DIFFERENT PUMP AMPLITUDE. ....................... 20

FIG. 2.11 INVERSION DISTRIBUTION IN SATURABLE ABSORBER FIBER UNDER DIFFERENT PUMP AMPLITUDE. ...... 21

FIG. 2.12 PEAK POWER, PULSE WIDTH, AND PULSE ENERGY FOR LASER WITH DIFFERENT GAIN FIBER LENGTH, PUMP ABSORPTION RATIO, AND PUMP AMPLITUDE.

FIG. 2.13 PEAK POWER, PULSE WIDTH, AND PULSE ENERGY FOR LASER WITH DIFFERENT SA FIBER LENGTH, 1064NM ABSORPTION RATIO, AND PUMP AMPLITUDE.

FIG. 2.14 PEAK POWER, PULSE WIDTH, AND PULSE ENERGY FOR LASER WITH DIFFERENT GAIN FIBER CORE DIAMETER, ABSORBER FIBER CORE DIAMETER, AND PUMP AMPLITUDE.

FIG. 2.15 PEAK POWER, PULSE WIDTH, AND PULSE ENERGY FOR LASER WITH DIFFERENT LASER WAVELENGTH, OUTPUT COUPLER REFLECTIVITY, AND PUMP AMPLITUDE...... 
FIG. 3.1 SCHEMATIC DIAGRAM OF ALL-FIBER PASSIVELY Q-SWITCHED FIBER LASER WITH YB-DCF AS SATURABLE ABSORBER

FIG. 3.2 A) OSCILLOSCOPE TRACE OF THE Q-SWITCHING PULSES AT 100 KHZ RATE; AND B) A SINGLE PULSE WITH

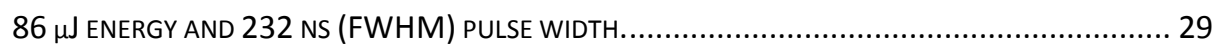

FIG. 3.3 OSCILLOSCOPE TRACES OF THE Q-SWITCHING PULSES AT A) 60 KHZ, AND B) $10 \mathrm{kHz} . \ldots \ldots \ldots \ldots \ldots . . . . . . . . .30$

FIG. 3.4 EMISSION SPECTRUM OF THE LASER AT A) 28 W OF POWER AMPLITUDE (LINE WIDTH IS 113 PM), AND

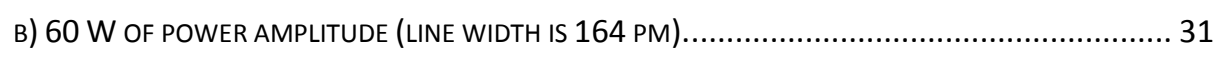

FIG. $3.5 \quad$ A) SPECTRAL LINE WIDTH VERSUS PUMP POWER AMPLITUDE; AND B) OUTPUT LASER POWER VERSUS

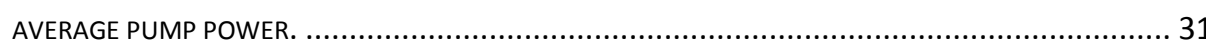

FIG. 3.7 A) PUMP DURATION VERSUS PUMP AMPLITUDE; AND B) LASER PULSE WIDTH AND PULSE ENERGY

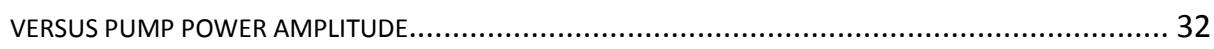

FIG. 3.8 PEAK POWER, PULSE WIDTH, AND PULSE ENERGY OF YB DOPED FIBER LASER WITH DIFFERENT ACTIVE

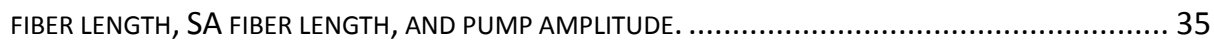

FIG. 3.9 PEAK POWER, PULSE WIDTH, AND PULSE ENERGY OF YB DOPED FIBER LASER WITH DIFFERENT SECONDARY GRATINGS WAVELENGTH, OC REFLECTIVITY, AND PUMP AMPLITUDE........................ 36

FIG. 4.1 GENERIC CONFIGURATION OF ALL-FIBER PASSIVELY Q-SWITCHED FIBER LASER WITH EXCESSIVE LONG YB-

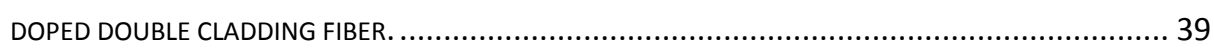

FIG. 4.2 A) OSCILLOSCOPE TRACE OF THE Q-SWITCHING PULSES AT 100 KHZ RATE; AND B) A SINGLE PULSE WITH

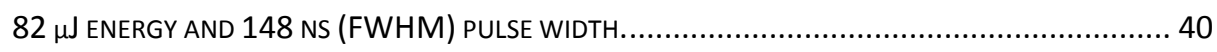

Fig. 4.3 OSCILLOSCOPE TRACES OF THE Q-SWITCHING PULSES AT A) 60 KHz, AND B) $10 \mathrm{kHz} . \ldots \ldots \ldots \ldots \ldots . . . . . . . .41$

FIG. 4.4 EMISSION SPECTRUM OF THE LASER AT A) 32 W OF POWER AMPLITUDE (LINE WIDTH IS 154 PM), AND

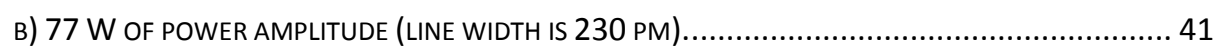

FIG. $4.5 \quad$ A) SPECTRAL LINE WIDTH VERSUS PUMP POWER AMPLITUDE; AND B) OUTPUT LASER POWER VERSUS AVERAGE PUMP POWER. 42

FIG. 4.6 A) PUMP DURATION VERSUS PUMP AMPLITUDE; AND B) LASER PULSE WIDTH AND PULSE ENERGY VERSUS PUMP POWER AMPLITUDE. 43

FIG. 4.7 PULSE WIDTH AND PULSE ENERGY VERSUS A) ACTIVE FIBER LENGTH, AND B) OUTPUT GRATING REFLECTION, OPERATED WITH PUMP POWER AMPLITUDE OF 50 W AT $100 \mathrm{kHz}$. 44

FIG. 4.8 A) SIMULATED OUTPUT PULSES FOR PROPOSED CONFIGURATION; B) RESIDUAL PUMP POWER ALONG GAIN FIBER FOR PROPOSED CONFIGURATION; C) SIMULATED OUTPUT PULSES WITH PUMP STRIPPER AT 8M; AND D) RESIDUAL PUMP POWER ALONG GAIN FIBER WITH PUMP STRIPPER AT 8M

FIG. 4.9 A) POPULATION INVERSION FOR THE LAST 1 M OF GAIN FIBER; B) CORRESPONDING SIMULATED PULSE TRAIN. 
FIG. 4.10 PEAK POWER, PULSE WIDTH, AND PULSE ENERGY OF YB DOPED FIBER LASER WITH DIFFERENT ACTIVE FIBER LENGTH, PUMP ABSORPTION RATIO, AND PUMP AMPLITUDE.......................................... 47

FIG. 4.11 PEAK POWER, PULSE WIDTH, AND PULSE ENERGY OF YB DOPED FIBER LASER WITH DIFFERENT LASER WAVELENGTH, OUTPUT COUPLER REFLECTIVITY, AND PUMP AMPLITUDE................................. 48

FIG. 4.12 PEAK POWER, PULSE WIDTH, AND PULSE ENERGY OF YB DOPED FIBER LASER WITH DIFFERENT CORE DIAMETERS AND PUMP AMPLITUDE. .. 49

Fig. B.1 TITANIUM SHEET METAL OF $136 \mu \mathrm{M}$ IS CUT AT SPEED OF $40 \mu \mathrm{M} / \mathrm{S}$ BY THE PROPOSED FIBER LASER WITH 2.8 W AVERAGE POWER, $100 \mathrm{KHZ}$ REPETITION RATE, 140 W PEAK POWER. 56

FIG. B.2 STAINLESS STEEL SHEET METAL OF $151 \mu \mathrm{M}$ IS DRILLED BY THE PROPOSED FIBER LASER WITH 5.0 W AVERAGE POWER, 100 KHZ REPETITION RATE, 240 W PEAK POWER.................................... 57 


\section{List of Appendices}

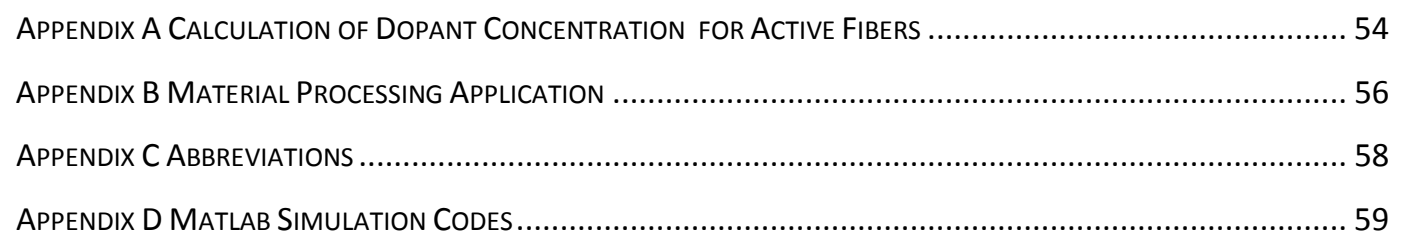




\section{Chapter 1}

\section{Introduction}

\subsection{Motivation}

Fiber lasers have attracted great interest in both research and industry in recent years. Fiber has a high surface area to volume ratio which provides efficient cooling that allows the generation of ultra high power without external active cooling. When using single mode gain and delivery fiber, very high quality optical beam can be produced at the output. Moreover, fiber cladding as the shielding that protects the laser in the core is highly resistive to vibration, almost immune from electromagnetic interference and toxic chemical agents. This enables fiber lasers to provide a readily solution in many harsh environment applications and a rather long lifetime. In addition, thin fiber is also very flexible. It can be easily coiled and packaged into a small module.

Pulsed lasers are usually preferred in many applications, including micromachining, material processing, second harmonic generation, and laser sensing. In general, There are two approaches to enable pulsed operation, modelocking and Q-switching. The basis of mode-locking is to induce a fixed phase relationship among the longitudinal modes in the laser's resonant cavity. Thus, constructive or destructive interference among these modes leads the laser producing a steady train of pulses. These pulses could be of extremely short duration on the order of picoseconds or femtoseconds depending on the design configuration. Q-switching, on the other hand, is to introduce a high loss before lasing in the cavity, i.e. a small Q factor. Therefore, force the gain medium to build up a relatively high population inversion, thus, a high gain to match up with the high loss. When the loss has been removed suddenly, i.e. switching the $\mathrm{Q}$ factor to a higher value, lasing starts rapidly releasing the stored energy in the 
gain medium. This results in a short pulse incorporating that energy, and thus a high peak power. Q-switching enables the generation of high peak power or high pulse energy with moderate pump power.

Recently, Q-switched fiber laser has drawn increasing research attentions. Different techniques have been implemented to achieve Q-switching, either actively or passively. Actively Q-switched fiber lasers have been realized by mechanically controlling the reflection spectrum of fiber Bragg gratings (FBG) output coupler. One mechanism to achieve this is using Acousto-optic modulators. Q-switched laser pulses of $10 \mathrm{~W}$ peak powers, $82 \mathrm{~ns}$ pulse width at repetition rate of up to $62.5 \mathrm{kHz}$ were obtained [1]. Alternatively, using magnetostrictive transducers, Q-switched pulses of more than $1 \mathrm{~W}$ peak power with a pulse repetition rate varied from $1 \mathrm{~Hz}$ to $125 \mathrm{kHz}$ were obtained in [2]. Piezoelectric actuators have been used in [3], and pulses with peak power of 530 $\mathrm{mW}$ at $3 \mathrm{kHz}$ were obtained. However, all these techniques require the assembly of either a bulk or complex modulator with the fiber laser. A more economic and compact approach is to replace the external modulator by saturable absorber. Lasers employ such a technique are referred as passively Q-switched lasers. Crystal saturable absorber is not suitable for all-fiber design. On the other hand, semiconductor saturable mirror (SESAM) allow all-fiber design, but it is vulnerable to high optical power, thus limiting the output power [4]. New designs involve the usage of fiber saturable absorber (SA). The advantages of these designs are SA's ability to hold enormous gain excited in the gain fiber from lasing and their high damage threshold for high-power Q-switched pulses. In [5], erbium (Er)-doped active fiber paired with thulium (Tm)-doped fiber absorber to generate Q-switched pulse with peak power of $7 \mathrm{~kW}$, pulse energy of $0.35 \mathrm{~mJ}$, pulse duration of $50 \mathrm{~ns}$, and repetition frequency at about $2 \mathrm{kHz}$. The energy level it produces can be employed in material processing, such as marking, trimming, and micromachining. 
Designs of passively Q-switched fiber lasers with fiber saturable absorber usually need to consider a proper relaxation mechanism of the excited state of SA. In general, there are four approaches to do so: choose a SA with non-radiative relaxation of excited state, induction of own lasing for SA, using highly doped SA, or use active fiber as SA with smaller mode-field areas.

Some rare-earth elements when doped in SA exhibit non-radiative decay from the excited state, such as samarium (Sm). The excited state lifetime for those absorbers is very short. For example, samarium has a life time less than 5 ns [6]. Q-switched ytterbium (Yb)-doped fiber laser with Sm-doped SA was demonstrated in [7-9]. In their demonstration, a steady pulse train with pulse duration of $650 \mathrm{~ns}$, repetition rate of about $130 \mathrm{kHz}$, peak power of $30 \mathrm{~W}$, and pulse energy of $20 \mu \mathrm{J}$ was generated from a $6 \mathrm{~W}$ pump source. Optimization of the absorber length could lead to high pulse energy.

When an absorption band of the doped ion corresponds to the radiative transition, excited state lifetime can be long. Thus, continuous wave (CW) operation or Qswitching with long pulse duration would be expected. Ref. [10] suggests a method to solve this problem by using Bismuth (Bi)-doped fiber as SA which has a long excited state lifetime of about $1 \mathrm{~ms}$. The authors separate Bi-doped SA in its own cavity with a pairs of high reflective FBGs at $1160 \mathrm{~nm}$, which is around the peak emission cross-section of Bi-ion. The laser generates pulses with peak power of 65W, pulse energy of $0.1 \mathrm{~mJ}$, minimum pulse duration of $1 \mu \mathrm{s}$, and repetition rate of 10-100 kHz.

A phenomenon of clustering is appeared when silica doped with high concentration of rare-earth ions. The clustered ion pair, upon excitation, would transfer one's energy to another; therefore, one undergoes the non-radiative decay to the ground state, while the other one undergoes a transition to a higher energy level. This phenomenon has an influence on laser dynamic, too. An Er-doped fiber laser design based on the heavily doped fiber absorber was described in [5]. Double clad Er-doped active fiber was paired with SA heavily doped with Tm. 
The concentration in SA was estimated to be $2 \times 10^{26} \mathrm{~m}^{-3}$. The laser produced pulse with repetition rate of $2 \mathrm{kHz}$, pulse duration of 50ns, pulse energy of $0.35 \mathrm{~mJ}$, and peak power of $7 \mathrm{~kW}$.

Another technique used to rapidly relax excited state ions in SA is to introduce mismatch of the mode-field areas between the active fiber and the SA. Rare-earth doped fiber usually exhibit absorption in the oscillation range as well. However, the absorption cross-section is rather smaller than the emission. To solve this problem, it is possible to induce a difference of power density in the absorber compared to the active fiber. A fiber absorber with the mode field area smaller than the active fiber can be chosen to achieve this goal. Although additional optical losses will be introduced by splicing, the power density can be increased in the fiber absorber. This technique was implemented in [11]. Two pieces of Erdoped fibers were used. One acts as active fiber had a mode field diameter of 14.5 $\mu \mathrm{m}$ and the other one is $6.5 \mu \mathrm{m}$. Laser was pumped into the core with maximum power of $280 \mathrm{~mW}$. Q-switched pulses were successfully produced with repetition rate of $0.25-1 \mathrm{kHz}$, pulse energy in the range of 8.3-6.0 $\mu \mathrm{J}$, and the pulse duration from 80 to 320 ns.

\subsection{Outline}

The configuration of Yb-doped passively Q-switched fiber laser with a Sm-doped fiber as SA is presented in chapter 2. A detailed theoretical model is developed to predict the uneven distribution of population inversion in both gain fiber and SA fiber. Numerically simulations also show the output characteristics versus pump power.

In chapter 3, configuration with Yb-doped fiber as SA is demonstrated and higher pulse energy is achieved. This configuration encloses SA fiber with a secondary cavity and also provide large mode field ratio between gain fiber and SA fiber. A theoretical model is developed to simulate the passive Q-switching for this configuration. 
A high peak power passively Q-switched Yb-doped fiber laser is described in chapter 4. An excessive long Yb-doped double-cladding fiber is used in the configuration. Since the fiber has large pump absorption, the residual pump power after propagating a certain distance in the fiber is negligible and of the remaining fiber could be considered as SA. Theoretical model is developed and numerical simulation provides solid proof for this mechanism. Q-switched pulses have been successfully demonstrated through experiment.

Chapter 5 summarizes the whole thesis. Remaining issues that might be interesting for future investigation are also discussed. 


\section{Chapter 2}

\section{All-fiber passively Q-switched fiber laser with Sm-}

\section{doped fiber saturable absorber}

\section{$2.1 \quad$ Introduction}

Previous efforts on passively Q-switched fiber laser have used bulk saturable absorbers, such as $\mathrm{Cr}^{4+}$ :YAG in [12], producing pulses with $25 \mu \mathrm{J}$ energy and 2.7 ns duration, which require external free-space optics coupling light into and out of the fiber. Recently implementation of rare-earth doped fiber as saturable absorber eliminates the bulk element and allows the design of true monolithic all-fiber laser. The first demonstration of such kind used a Yb-doped double-cladding fiber of 20 $\mathrm{m}$ long as active fiber paired with a $10 \mathrm{~cm}$ long Sm-doped SA fiber. [7], to generate pulses with $19 \mu \mathrm{J}$ energy and 650 ns duration. However the pulse to pulse stability was poor. Tsai et al. reported an Er-doped fiber laser using a piece of unpumped Er-doped fiber as an SA which produced $8.3 \mu \mathrm{J}, 80$ ns pulses [11]. The same group lately successfully demonstrated an all-fiber Q-switched laser with mode-field-area mismatching Yb-doped gain fiber and SA fiber which generated $2.8 \mu \mathrm{J} 280 \mathrm{~ns}$ pulses [13]. Their research clearly demonstrated the feasibility of achieving stable Q-switched pulses in all-fiber configuration. However these lasers, so far, produced only relatively low pulse energy and low average power, far from the requirements for laser material processing.

In this chapter, we present a new configuration for passively Q-switched all-fiber laser. As well as for other Q-switching laser design, the SA fiber must be properly selected, such that its absorbing band matches the laser oscillation wavelength. We select Sm-doped silica fiber as SA to match with Yb-doped double-cladding gain fiber to oscillate at $1064 \mathrm{~nm}$. The laser successfully produces stable pulses of 
$68 \mu \mathrm{J}$ pulse energy with $210 \mathrm{~ns}$ duration. Wavelength at $1064 \mathrm{~nm}$ is widely used in material processing industry and therefore can be readily adopted. Another reason for this wavelength is that its second and third harmonic generation at 532nm and 355nm, are also important, having applications in silicon wafer cutting and inscribing. In addition, Sm-doped fiber provides fast non-radiative decay, which is essential for high repetition application. The lifetime of the transition from upper level of this saturable absorber was evaluated to be less than 5 ns [6].

Theoretical model of the passively Q-switched fiber laser is also presented in section 2.4, including the rate equations for both gain fiber and SA. In order to accurately describe the inversion density, pump power and radiation power along the fiber, the traveling wave method is implemented. Since the proper inclusion of amplified spontaneous emission (ASE) has been suggested to be important to fully model the operation of a passively Q-switched fiber laser [14], wavelength span from $1030 \mathrm{~nm}$ to $1100 \mathrm{~nm}$ with its corresponding emission and absorption cross sections is incorporated in the simulation.

\subsection{Experiment setup}

The laser is monolithic in design with all connections spliced, as shown in Fig 2.1. The pump light from three diodes, each rated at $25 \mathrm{~W}, 975 \mathrm{~nm}$, is coupled into the large-mode area (LMA) gain fiber through a $(3+1)$ x1 power combiner with a 0.5 $\mathrm{dB}$ insertion loss per pump port. The fiber from input and output port of the combiner has a 10/125 $\mu \mathrm{m}$ core/cladding diameter. The $4.5 \mathrm{~m}$ long Yb-doped gain fiber from Nufern has a nominal cladding absorption coefficient of $5.4 \mathrm{~dB} / \mathrm{m}$ at $975 \mathrm{~nm}$ and core/cladding diameter of 15/130.

The laser cavity consists of a highly reflective fiber Bragg grating (HR-FBG) of more than 99\% reflectivity and $0.28 \mathrm{~nm}$ bandwidth at $1064 \mathrm{~nm}$ which was spliced at the input port of the combiner, and the gain fiber was spliced at the output port of the combiner. An output coupler FBG (OC-FBG) of $4 \%$ reflectivity and 0.30 
$\mathrm{nm}$ bandwidth was spliced at the other end of the gain fiber. The OC-FBG was fabricated on the fibers whose core/cladding diameters also match the gain fiber.

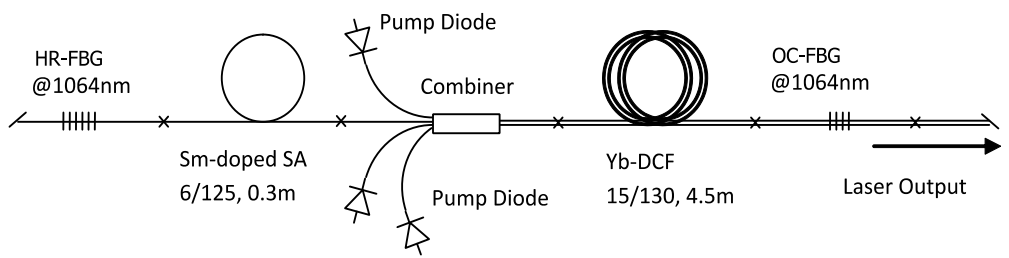

Fig. 2.1 Configuration diagram of passively Q-switched all-fiber laser incorporating mode field mismatching.

Within the laser cavity, a piece of Sm-doped single-mode fiber with a core diameter of $6.3 \mu \mathrm{m}$ and numerical aperture (NA) of 0.14 , was inserted. The absorption of the saturable absorber fiber at $1064 \mathrm{~nm}$ is estimated to be $8 \mathrm{~dB} / \mathrm{m}$. Since the actual core diameter of the gain fiber is $15 \mu \mathrm{m}$, the core area ratio of the gain to SA fiber reaches 5.7:1. When the $975 \mathrm{~nm}$ pump excites the population inversion in Yb-doped gain fiber, the ASE is confined in the core and propagating in both directions and thus bleaches the SA fiber. A large area ratio will help to store more power in gain fiber before bleaching the SA to start Q-switching. The use of the single-cladding SA fiber will not lead to the loss of pump light in this configuration.

The power supply to the pump diodes can operate in pulsed modes and its duty cycle can be adjusted. The repetition rate can also be adjusted up to $100 \mathrm{kHz}$. The fiber of the OC-FBG was spliced to an angle-polished connector with a same $10 / 125 \mu \mathrm{m}$ core/cladding diameter. To measure the Q-switched pulse train and laser emission spectrum, the laser output was imaged by a lens of $62 \mathrm{~mm}$ focal length onto the connector of a patch cord which was directed into either an optical spectral analyzer or a fast photodiode with $1 \mathrm{GHz}$ in bandwidth.

\subsection{Results and discussion}

The laser was tested at the repetition rate varying from $10 \mathrm{kHz}$ to $100 \mathrm{kHz}$. The pump current and duration were adjusted to obtain stable Q-switched pulses in 
both amplitude and time. When the laser was pumped at the repetition rate of 100 kHz, Q-switched pulse train such as shown in Fig 2.2(a), was obtained. The peak amplitude variation was about $3.27 \%$ in standard deviation and the pulse duration varied at $1.73 \%$. Similar pulse amplitude stability and low pulse jitter were observed at other repetition rates. The single Q-switched pulse is shown in Fig 2.2(b) which has a slightly asymmetric shape with a steep rising edge and a slower tailing edge.
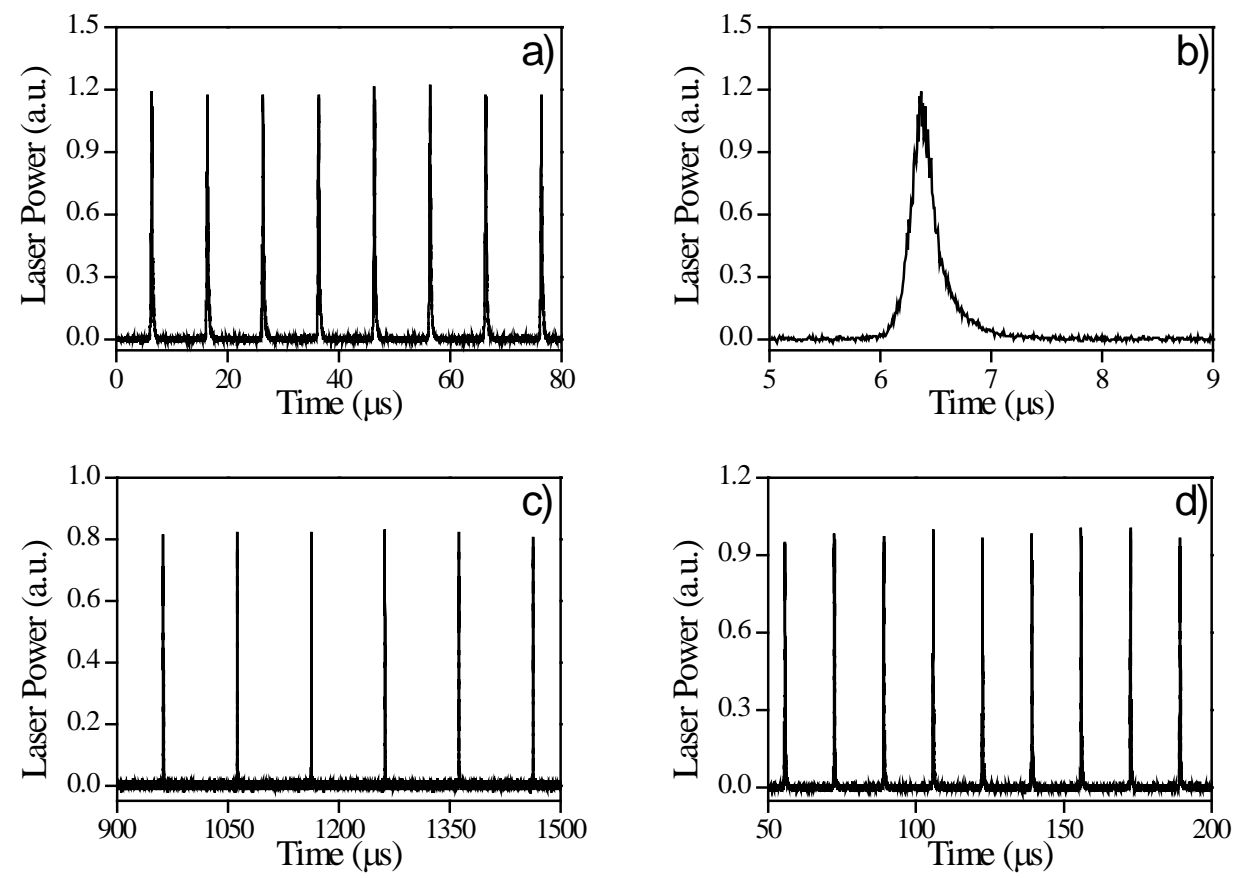

Fig. 2.2 (a) Oscilloscope trace of the Q-switching pulses at $100 \mathrm{kHz}$ rate;

(b) a single pulse with $68 \mu \mathrm{J}$ energy and $210 \mathrm{~ns}$ (FWHM) pulse duration.

(c) Oscilloscope traces of the Q-switching pulses at repetition rate of $10 \mathrm{kHz}$, and (d) at $60 \mathrm{kHz}$.

When the laser was Q-switched at $100 \mathrm{kHz}$, the average output power of $6.8 \mathrm{~W}$ was obtained that gave the energy per pulse of $68 \mu \mathrm{J}$, more than 35 times the energy of SESAM Q-switched laser pulse and 20 times higher than what reported in [13]. The peak power of $324 \mathrm{~W}$ was obtained at the pulse width of $210 \mathrm{~ns}$. At lower repetition rates, such as $10 \mathrm{kHz}$ and $60 \mathrm{kHz}$, stable pulses were also obtained, as shown in Fig 2.2(c) and (d). 
When the laser was operated at $100 \mathrm{kHz}$, the duty cycle of the pump was kept at $\sim 31 \%$, as shown in Fig 2.3(a). This indicates that the laser had the potential to go to a higher repetition rate, and thus, further increase its average output power. One important feature of this laser is its adjustable repetition rate by changing the repetition rate of the pump laser. A laser with a variable pulse rate but same pulse shape will make it versatile for various applications.
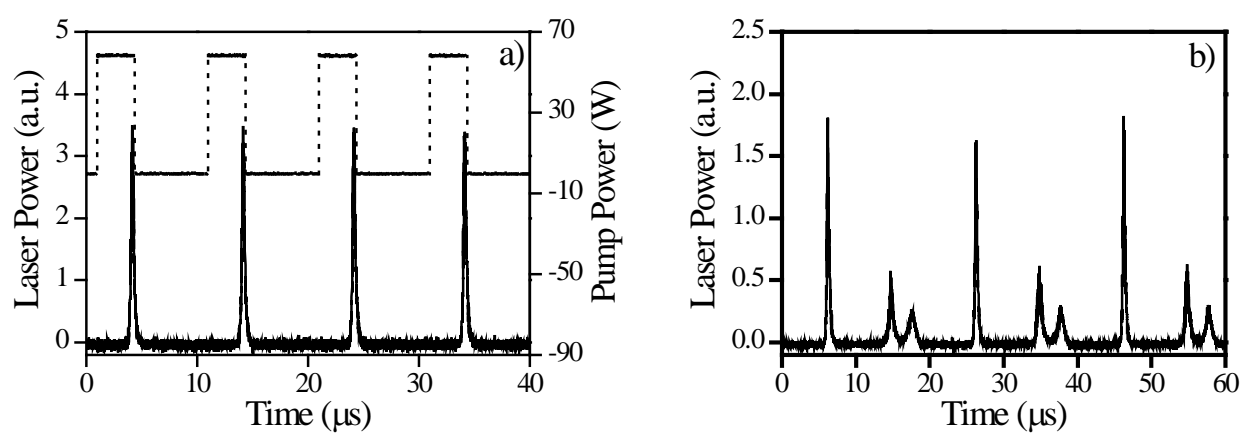

Fig. 2.3 (a) A comparison of pulsed pump power (dots) and output laser pulses (solid) at $100 \mathrm{kHz}$; (b) A Q-switching pulse train with relaxation oscillation pulses pumped at $100 \mathrm{kHz}$ and above 31\% duty-cycle than normal operation condition.

In general, the pump duration should be kept as long as possible to store more energy in the gain medium before Q-switching. However excessive long pump time will trigger relaxation oscillation as illustrated in Fig 2.3(b) which was obtained at the same pump amplitude of $60 \mathrm{~W}$, however, longer duty cycle than what used in Fig 2.3(a). After Q-switched pulse the gain fiber continues to accumulate energy which led the generation of the relaxation pulses with less energy even after the pump is turned off. When that happened, the Q-switched pulses become unstable; their amplitudes fluctuate and pulse jitter increases. In our experiment, the pump was turned off before the onset of the after-pulses. For example, at the $100 \mathrm{kHz}$ repetition rate, the pump duration was kept at less than $3.1 \mu$ s which prevented the start of the after-pulses. When the laser was CW pumped, Q-switched pulses and relaxation pulses were mixed together that made whole pulse train chaotic. 

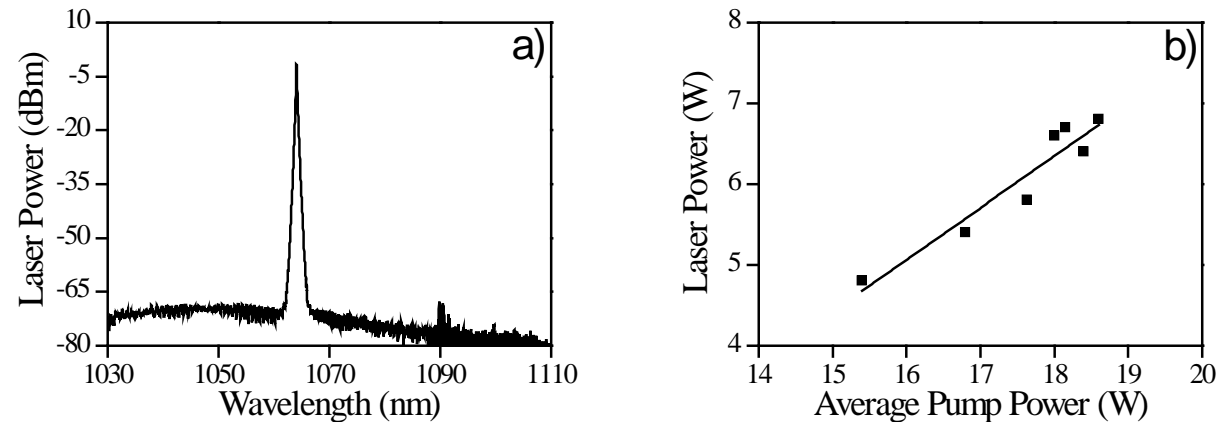

Fig. 2.4 (a) Emission spectrum of the laser at $100 \mathrm{kHz}$; and

(b) Average laser power vs. average pump power operated at $100 \mathrm{kHz}$.

The laser emission spectrum, at $1064.05 \mathrm{~nm}$ with a $0.161 \mathrm{~nm}$ bandwidth, is plotted in Fig 2.4(a), which shows an excellent optical signal to noise ratio of $\sim 70$ $\mathrm{dB}$ and a narrow bandwidth. The average pump power was measured after the combiner when pump laser operated at the pulse mode. The corresponding laser output power was measured as a function of pump power as shown in Fig. 2.4(b). The laser efficiency is indicated by the slope of the linear fitting line and is calculated to be $64 \%$, which is far exceeding the slope efficiency of $9 \%$ reported in [15]. There is no trend of saturation shown in Fig. 2.4(b), indicating the potential of producing higher laser power without sacrificing efficiency. Further power scale-up can be made by increasing the maximum power of each pump diode, or coupling more diodes with a $(1+6)$ x1 power combiner.

For a given SA length of $30 \mathrm{~cm}$ at the pump frequency of $100 \mathrm{kHz}$, we measured the pump duration as a function of pump power amplitude. The pump duration was carefully selected to avoid the occurrence of after-pulses: it was increased firstly until a weak after-pulse was observed, and then decreased with a small decrement step until the after-pulse was flattened. This procedure guarantees a stable pulse train at the highest pulse energy without an after-pulse. The pump duration decreased from $5.5 \mu$ s to $3.1 \mu$ s with the increase of pump amplitude from $28 \mathrm{~W}$ to $60 \mathrm{~W}$, as shown in Fig 2.5(a). The corresponding laser pulse width and pulse energy were plotted in Fig 2.5(b), which showed the pulse width decreases and the pulse energy increases as the pump amplitude increases. The 
results as such are expected; as the pump amplitude increases, the ASE in the gain fiber takes less time to build up and to bleach the SA. Moreover, since the pump light intensity was higher, once the SA was bleached, the higher population inversion in the gain fiber was depleted faster which resulted in shorter pulse width and higher pulse energy extracted in the Q-switching process. The high power intensity in the cavity improved the stimulated emission process. Therefore, an effective way to increase the peak power and pulse energy is to increase the amplitude of the pump power. The slope of the pulse energy plotted in Fig 2.5(b) shows slightly roll-off at pump amplitude beyond $50 \mathrm{~W}$, indicating that higher pump amplitude may slightly increase the pulse energy.
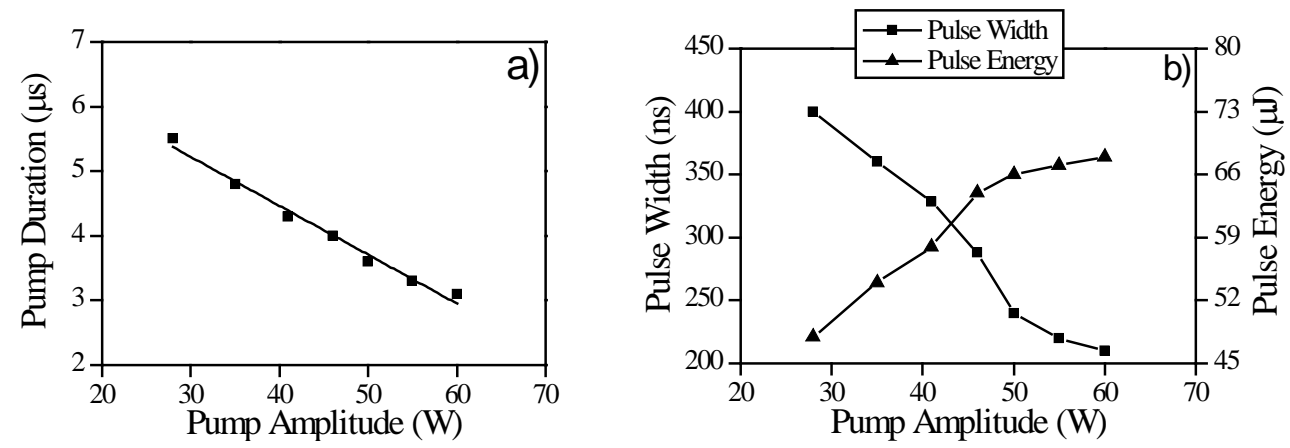

Fig. 2.5 (a) Pump duration vs. pump amplitude; and (b) laser pulse width and pulse energy versus pump power amplitude. The laser is operated at $100 \mathrm{kHz}$ with a $30 \mathrm{~cm}$ long of SA.

In order to determine the effects of SA length on the laser performance, we adjusted the pump duration for achieving stable Q-switch at three different SA lengths of $50 \mathrm{~cm}, 40 \mathrm{~cm}$ and $30 \mathrm{~cm}$, respectively. We found that shorter SA fiber decreased the pump duration, reduced only slightly the laser pulse width and increased the pulse energy by about $15 \%$. We considered that the threshold for ASE to bleach the SA decreases with a shorter of SA fiber, which led to the shorter pump duration required to reach the ASE threshold. Due to the fast decay and high loss of Sm-doped fiber, the shorter the SA, the less energy lost in propagating through the SA which led to the increase of output pulse energy. However, if higher pump power is available, longer SA fiber is preferred to force 
the gain fiber to reach higher population inversion and therefore produce pulses with higher energy.

\section{$2.4 \quad$ Theoretical model}

Travelling wave model is proper for a long cavity and is able to provide reasonable prediction of spectrum, power, and dopant inversion along the fiber. As shown in Fig 2.6, optical power carried by photon flux travelling back and forth in the laser cavity. The power is amplified along its path in gain fiber, while attenuated in SA fiber. The output power is the result of transmittance of OC-FBG.

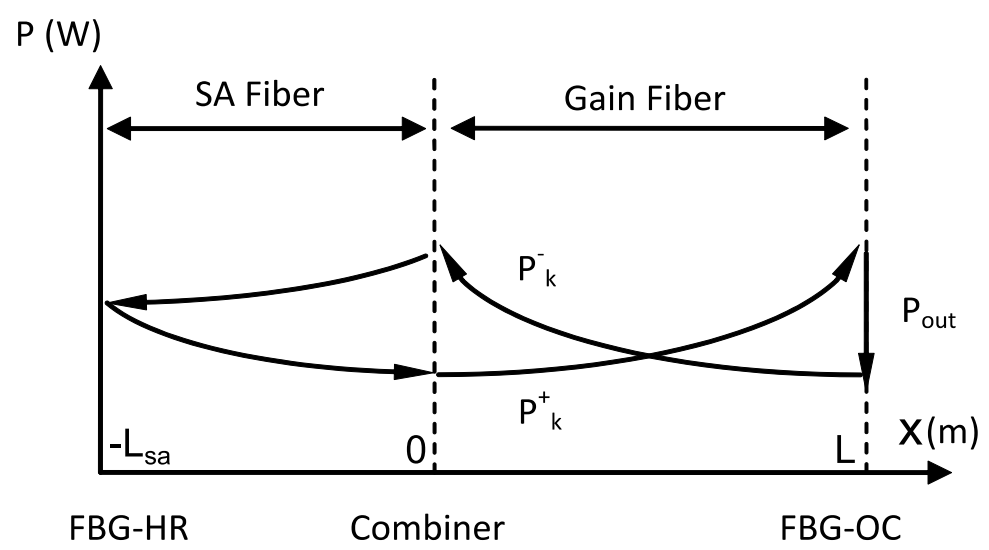

Fig. 2.6 Travelling wave model for the passively Q-switched fiber laser with SA fiber.

We assume that active ions are doped evenly along $\mathrm{x}$-axis and along the core radius in the active fiber. The photon flux is also assumed to be evenly spread in radial direction in the fiber core. The Rayleigh-scattering effect is omitted for simplicity [16]. In gain fiber, the governing rate equations are given by

$$
\begin{aligned}
& N_{0}=N_{1}(x, t)+N_{2}(x, t) \\
& \frac{\partial N_{2}(x, t)}{\partial t}=\frac{\Gamma_{p} \lambda_{p}}{h c A_{c o}}\left[\sigma_{a p} N_{1}(x, t)-\sigma_{e p} N_{2}(x, t)\right] P_{p}^{+}(x, t) \\
& +\sum_{k} \frac{\Gamma_{k} \lambda_{k}}{h c A_{c o}}\left[\sigma_{a k} N_{1}(x, t)-\sigma_{e k} N_{2}(x, t)\right]\left[P_{k}^{+}(x, t)+P_{k}^{-}(x, t)\right] \\
& -\frac{N_{2}(x, t)}{\tau}
\end{aligned}
$$




$$
\begin{aligned}
& \frac{d P_{p}^{+}(x, t)}{d x}=\frac{\partial P_{p}^{+}(x, t)}{\partial x}+\frac{1}{v_{p}} \frac{\partial P_{p}^{+}(x, t)}{\partial t} \\
& =\Gamma_{p}\left[\sigma_{e p} N_{2}(x, t)-\sigma_{a p} N_{1}(x, t)\right] P_{p}^{+}(x, t)-\alpha_{p} P_{p}^{+}(x, t) \\
& \frac{d P_{k}^{ \pm}(x, t)}{d x}= \pm \frac{\partial P_{k}^{ \pm}(x, t)}{\partial x}+\frac{1}{v_{k}} \frac{\partial P_{k}^{ \pm}(x, t)}{\partial t} \\
& =\Gamma_{k}\left[-\sigma_{a k} N_{1}(x, t)+\sigma_{e k} N_{2}(x, t)\right] P_{k}^{ \pm}(x, t) \\
& -\alpha_{k} P_{k}^{ \pm}(x, t)+N_{2}(x, t) \xi_{k}
\end{aligned}
$$

where $N_{0}$ is the doping concentration in the gain fiber, $N_{1}$ and $N_{2}$ are the ground and excited state population densities, $P_{p}^{+}$is the pump power (the superscript ' \pm ' correspond to the forward and backward propagations, respectively), $\lambda_{p}$ is the pump wavelength, $\lambda_{k}$ is the indexed ASE wavelength, $\sigma_{a p}, \sigma_{e p}, \sigma_{a k}, \sigma_{e k}$ and are the absorption and emission cross-sections for the pump wavelength and indexed signal wavelength, $\Gamma_{p}$ and $\Gamma_{k}$ are the power confinement factor for the pump and the emissions,

$$
\begin{aligned}
& \Gamma_{p}=\frac{d_{\text {core }}^{2}}{d_{\text {cladding }}^{2}} \\
& \Gamma_{k}=1-\exp \left(-\frac{2 d_{\text {core }}^{2}}{4 \omega^{2}}\right) \\
& V=\frac{2 \pi d_{\text {core }} N A}{2 \lambda_{k}} \\
& \omega=\frac{d_{\text {core }}}{2}\left(0.65+1.619 V^{-\frac{3}{2}}+2.879 V^{-6}\right)
\end{aligned}
$$

$\alpha_{p}$ and $\alpha_{k}$ are the dissipative optical loss for the pump and the emissions, $v_{p}$ and $v_{k}$ are the velocity for the pump and the emissions, $\tau$ is the excited state lifetime, $A_{c o}$ is the area of the fiber core, $h$ is the Planck constant, $c$ is the speed of the light, and $\xi_{k}$ represents the spontaneous emission coefficient and is given by 


$$
\xi_{k}=M \Gamma_{k} \sigma_{e k} \frac{h c^{2}}{\lambda_{k}^{3}} \Delta \lambda_{k}
$$

where $M$ is the number of transverse modes, which is 2 for $\mathrm{LP}_{01}$ single-mode, and $\Delta \lambda_{k}$ is the step used in the discrete integration of wavelength.

Since the pump power is never reflected back to interact with SA fiber, terms with pump power are omitted in the analysis of SA fiber. The Sm-doped fiber does not have emission band in the oscillation band of the laser; therefore, the equation (2.4) is modified to omit the emission cross section coefficient and spontaneous emission. We can then write rate equations for Sm-doped SA fiber as,

$$
\begin{aligned}
& N_{0}^{s a}=N_{1}^{s a}(x, t)+N_{2}^{s a}(x, t) \\
& \frac{\partial N_{2}^{s a}(x, t)}{\partial t}=\sum_{k} \frac{\Gamma_{k}^{s a} \lambda_{k}}{h c A_{c o}^{s a}}\left[\sigma_{a k}^{s a} N_{1}^{s a}(x, t)\right]\left[P_{k}^{+}(x, t)+P_{k}^{-}(x, t)\right] \\
& -\frac{N_{2}^{s a}(x, t)}{\tau_{s a}} \\
& \frac{d P_{k}^{ \pm}(x, t)}{d x}= \pm \frac{\partial P_{k}^{ \pm}(x, t)}{\partial x}+\frac{1}{v_{k}^{s a}} \frac{\partial P_{k}^{ \pm}(x, t)}{\partial t} \\
& =-\Gamma_{k}^{s a}\left[\sigma_{a k}^{s a} N_{1}(x, t)\right] P_{k}^{ \pm}(x, t)-\alpha_{k}^{s a} P_{k}^{ \pm}(x, t)
\end{aligned}
$$

where the superscript 'sa' indicates the parameters for the absorber fiber. $\Gamma_{k}^{s a}$ is calculated follow the same equation for gain fiber except using the core diameter for SA fiber.

With the following boundary conditions, the above system of equations can be numerically solved and simulated using the finite-difference method [17].

$$
\begin{aligned}
& P_{p}^{+}(0, t)=P_{\text {pump }}(t) \\
& P_{k}^{-}(L, t)=R_{o c} P_{k}^{+}(L, t) \\
& P_{k}^{+}\left(0^{+}, t\right)=(1-\eta) P_{k}^{+}\left(0^{-}, t\right) \\
& P_{k}^{+}\left(-L_{s a}, t\right)=R_{H R} P_{k}^{-}\left(-L_{s a}, t\right)
\end{aligned}
$$




$$
P_{k}^{-}\left(0^{-}, t\right)=(1-\eta) P_{k}^{-}\left(0^{+}, t\right)
$$

where $\eta$ is the sum of splice loss plus insertion loss between gain fiber and SA fiber. Note that the length of all the gratings and combiner are neglected for simplicity. $R_{o c}$ and $R_{H R}$ are reflectivity of OC-FBG and HR-FBG, respectively. Since we choose $\Delta \lambda_{k}$ of $2 \mathrm{~nm}$ in the simulation, which is much wider than the bandwidth of both FBGs, the respective reflectivity for other than the designed wavelength is 0 .

The laser output power is the integral of power of all wavelengths being coupled out at the output pigtail end. The length of output pigtail is neglected in this case.

$$
P_{\text {output }}(t)=\left(1-R_{o c}\right) \int_{k} P_{k}^{+}(L, t)
$$

In this simulation, we use Yb-doped double cladding fiber from Nufern (LMAYDF-15/130-VIII) as gain fiber. The nominal cladding absorption coefficient is $5.4 \mathrm{~dB} / \mathrm{m}$ at $975 \mathrm{~nm}$. The dopant concentration is estimated at $2.14 \times 10^{25} \mathrm{~m}^{-3}$ by using the method provided by Appendix A. CorActive Sm119 fiber is used as SA fiber with 6/125 core/cladding diameter and $8 \mathrm{~dB} / \mathrm{m}$ estimated absorption at $1064 \mathrm{~nm}$ in core. The dopant concentration for this fiber is estimated at $1.45 \times$ $10^{25} \mathrm{~m}^{-3}$. The spontaneous emission wavelength is considered spanning from $1030 \mathrm{~nm}$ to $1100 \mathrm{~nm}$, which gives total number of $\mathrm{k}=36$ channels. Other parameters used in the simulation are given in Table 2.1 as in Ref [18].

\begin{tabular}{|l|l|l|l|l|l|}
\hline Parameter & Value & Parameter & Value & Parameter & Value \\
\hline$\sigma_{e k}(\mathrm{k}=1064 \mathrm{~nm})$ & $0.35 \times 10^{-24} \mathrm{~m}^{2}$ & $N_{0}$ & $2.14 \times 10^{25} \mathrm{~m}^{-3}$ & $\alpha_{k}$ (for all k) & $0.005 \mathrm{~m}^{-1}$ \\
\hline$\sigma_{a k}(\mathrm{k}=1064 \mathrm{~nm})$ & $0.01 \times 10^{-24} \mathrm{~m}^{2}$ & $N_{0}^{s a}$ & $1.45 \times 10^{25} \mathrm{~m}^{-3}$ & $\alpha_{k}^{\text {sa }}($ for all $\mathrm{k})$ & $0.005 \mathrm{~m}^{-1}$ \\
\hline$\sigma_{a k}^{s a}(\mathrm{k}=1064 \mathrm{~nm})$ & $0.7 \times 10^{-25} \mathrm{~m}^{2}$ & $\tau$ & $0.84 \times 10^{-3} \mathrm{~s}$ & $\alpha_{p}$ & $0.005 \mathrm{~m}^{2}$ \\
\hline$\sigma_{a p}(\mathrm{p}=975 \mathrm{~nm})$ & $2.50 \times 10^{-24} \mathrm{~m}^{2}$ & $\tau_{s a}$ & $5 \times 10^{-9} \mathrm{~s}$ & $\mathrm{R}_{\mathrm{OC}}$ & $10 \%$ \\
\hline$\sigma_{e p}(\mathrm{p}=975 \mathrm{~nm})$ & $2.44 \times 10^{-24} \mathrm{~m}^{2}$ & $d_{c o r e}$ & $15 \times 10^{-6} \mathrm{~m}$ & $\mathrm{R}_{\mathrm{HR}}$ & $99.9 \%$ \\
\hline $\mathrm{L}$ & $4.5 \mathrm{~m}$ & $d_{c o r e}^{\text {sa }}$ & $6 \times 10^{-6} \mathrm{~m}$ & $\eta$ & 0.5 \\
\hline
\end{tabular}

Table 2.1 Simulation parameters in passively Q-switched fiber laser with Sm-doped SA. 
In the next section, we will show simulation results and discuss the dynamic in gain fiber as well as in SA fiber.

\subsection{Dynamic of passively Q-switched fiber laser}

Pulsed pump scheme is used in the simulation. The pump duration is program controlled to turn off at peak of each laser pulse. I conduct the simulation with different SA fiber length from $50 \mathrm{~cm}$ to $20 \mathrm{~cm}$ with different pump amplitude from $22 \mathrm{~W}$ to $94 \mathrm{~W}$ and pumping frequency from $10 \mathrm{kHz}$ to $100 \mathrm{kHz}$. We set the time resolution to $5 \mathrm{~ns}$ for all simulation to get sufficient details of evolving pulses. The pulse train is generated with high stability in both amplitude and temporal profile, except the first pulse. At the beginning of first pumping cycle, the dopant inversion in gain fiber is set to 0 . Since the pumping duration is less than the $\mathrm{Yb}$ lifetime, before the next consecutive pumping cycle, the dopant inversion could not restore to the original level. Thus, the first pulse is different from the following ones. The first pulse, (whose amplitude is $1.5 \%$ higher than the regular pulse) is excluded from the calculation of the laser peak power, pulse duration and pulse energy. We noticed that the peak power is reduced merely less than $0.5 \%$ as the pumping frequency increased from $10 \mathrm{kHz}$ to $100 \mathrm{kHz}$, which gives the theoretical evidence for a reliable tunable repetition rate fiber laser.

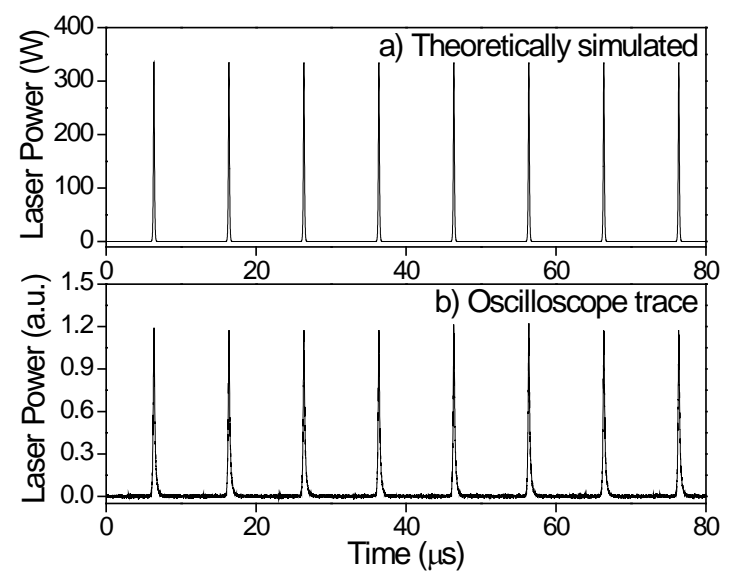

Fig. 2.7 a) Theoretically simulated Q-switching pulse trains at $100 \mathrm{kHz}$

b) Oscilloscope trace of the Q-switching laser under the same operation condition. 
The simulation comparing with the experimental obtained pulse trains are shown in Fig 2.7. In order to understand the dynamic for the evolution of pulses, a single pulse from the steady pulse train generated from such configuration with $30 \mathrm{~cm}$ long absorber fiber pumped with 70W of power is shown in Fig 2.8. The dotted lines are time reference at different moments of events. The time span $1 \mu \mathrm{s}$ from the start of pumping cycle $(\mathrm{t}=0)$ plus $2.2 \mu \mathrm{s}$, which gives sufficient details of dynamic in laser cavity.

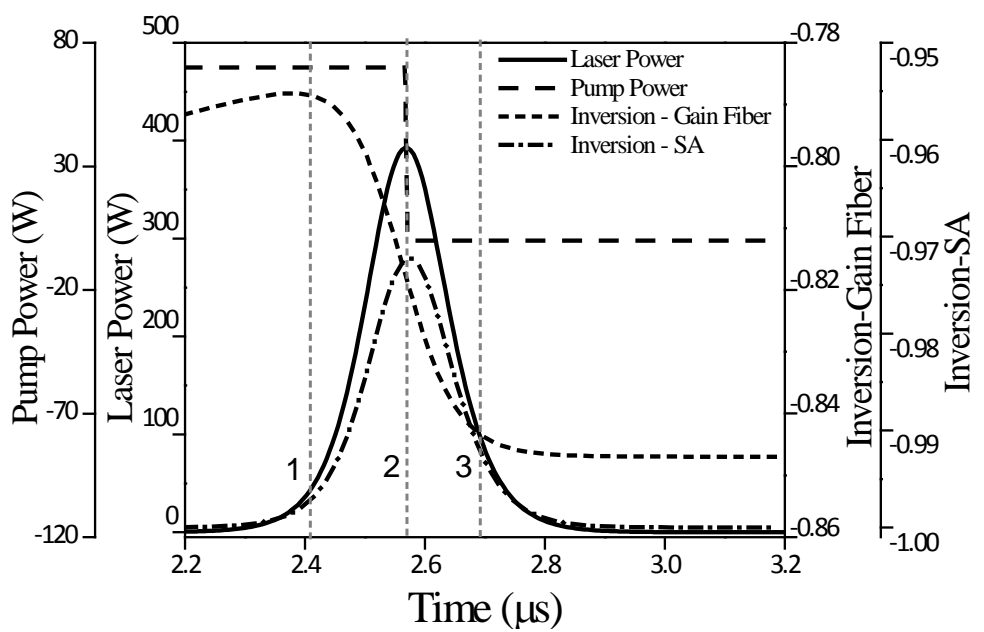

Fig. 2.8 A single pulse (solid) along with the corresponding pump power (loosely dashed), inversion in gain fiber (dashed), and inversion in SA (dash-doted) generated with $30 \mathrm{~cm} \mathrm{SA}$ and 70W pump amplitude.

During the first $2.2 \mu \mathrm{s}$, which is not shown in the plot, the pump power is steady at $70 \mathrm{~W}$, and the population inversion in gain fiber is increasing at a constant rate as shown before line 1 . The population inversion in gain fiber is increasing at a constant rate until about $2.4 \mu$ s (at line 1), and no significant optical power is shown at output. The ASE is dominating in the cavity during this process. The population inversion in SA fiber is brought to slightly higher than -1.

Line 1 is the onset of the lasing, while line 2 is when pulse reaches its peak. During the time from line 1 to line 2, the laser power grows dramatically with the decrease in population inversion in gain fiber. As the population inversion in gain fiber decreases, the gain is reducing until a critical level, marked by line 2, where 
the pulse reaches the peak and relaxation oscillation commences in the cavity. Continue pumping after this point will generate after-pulses and the overall efficiency will be degraded.

Note that the ratio of increasing rate of population inversion in SA fiber to the increase rate of the laser power remains constant and pulse reaches peak as the inversion reaches peak. It indicates that the population inversion in SA fiber is proportional to the number of photons in the cavity. From line 2 to line 3, the population inversion in gain fiber continues rapid decreasing, while the laser power is decreasing. After line 3, the optical power diminished, and the inversion in SA fiber restores to original and waits for the next pumping cycle. Note that the inversion in gain fiber slowly decreases with the rate of $1 / \tau$.

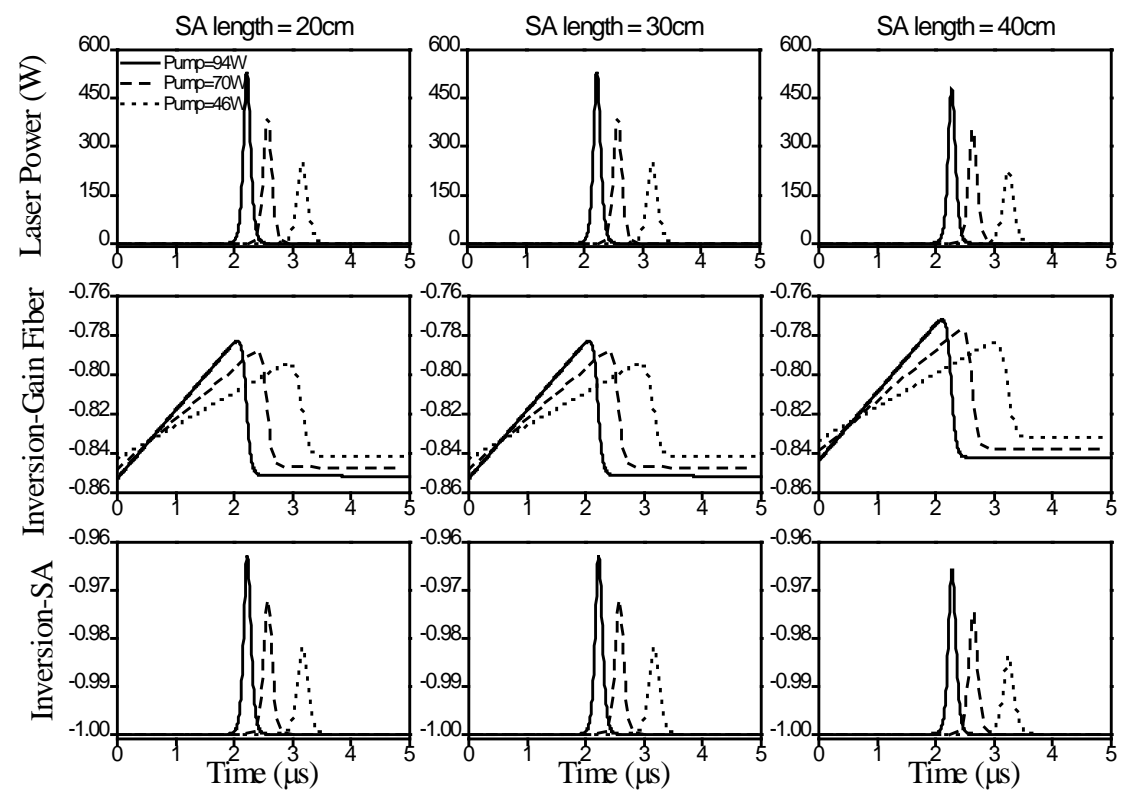

Fig. 2.9 Pulse shape, inversion in gain fiber, and inversion in SA generated with different SA length and pump amplitude.

Dynamic of different SA fiber length and pump amplitude are compared in Fig 2.9. When SA length is fixed, the time required to on-set of the pulse is reduced with the increasing of pump power. The increasing rate of population inversion in gain fiber is more rapid for higher pump amplitude before lasing, and decreasing rate is also slightly rapid. This leads to shorter pulse duration at output. The peak 
inversion in gain fiber is improved for higher pump amplitude, which leads to higher pulse energy and peak power. Note that when the SA length increases, the plot of inversion in gain fiber shifted upwards. It indicates that due to the total loss in the cavity increased, the inversion in gain fiber is improved to onset the pulse. However, the ASE level required to onset the pulse is also increased, results producing smaller pulse energy.

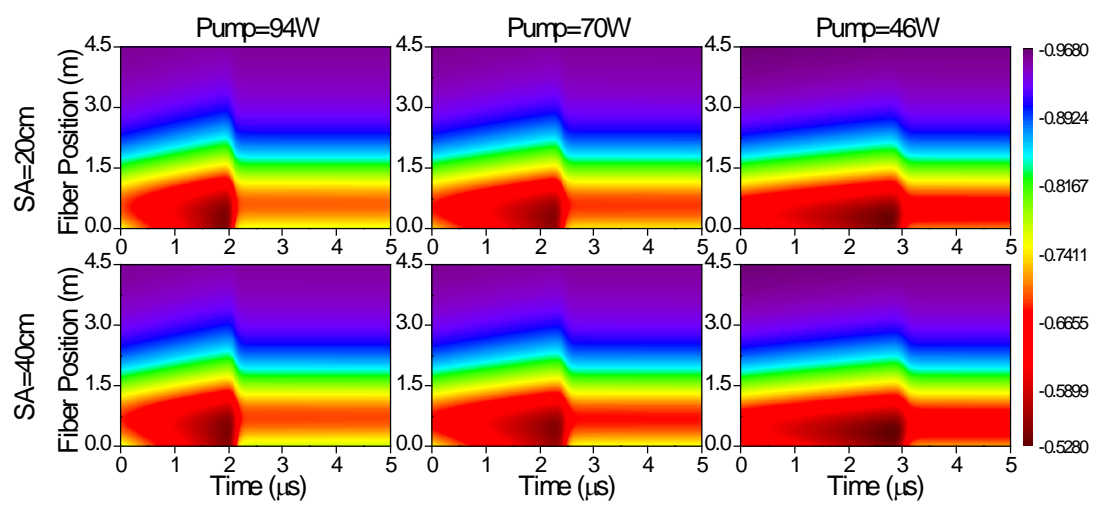

Fig. 2.10 Inversion distribution in active fiber under different pump amplitude.

Since fiber lasers usually employ a long cavity, the population inversion is unevenly distributed along the fiber for both gain fiber and SA fiber. It's interesting to study the nature of such phenomenon. The population inversion distribution is plotted in Fig 2.10 for gain fiber and Fig 2.11 for SA fiber. Different SA length and pump amplitude are compared. Please note that the power combiner and SA fiber are spliced at $\mathrm{x}=0$. Note that the highest population inversion along the gain fiber is not at the end where connected to the pump. This position shifted further away as increasing in pump amplitude and increasing in SA fiber length. Intuitively, this can be understood as when the inversion increased through pumping is fast in the small portion, where connected to the pump, the depleting rate of excited state is also fast through ASE and stimulated emission. Thus, the location with the highest value is shifted along the direction of pump power. 
The distribution of population inversion in SA fiber is shown in Fig. 2.11 to compare different absorber length, configurations, and pump amplitude. The distribution in Sm-doped absorber fiber is almost uniformly with slightly decreasing moving away from the connection with the gain fiber. The pumping time required to saturate the absorber is reduced by increasing the pump amplitude or decreasing the absorber length. The saturation threshold is increased by either increasing pump amplitude or decreasing absorber length.

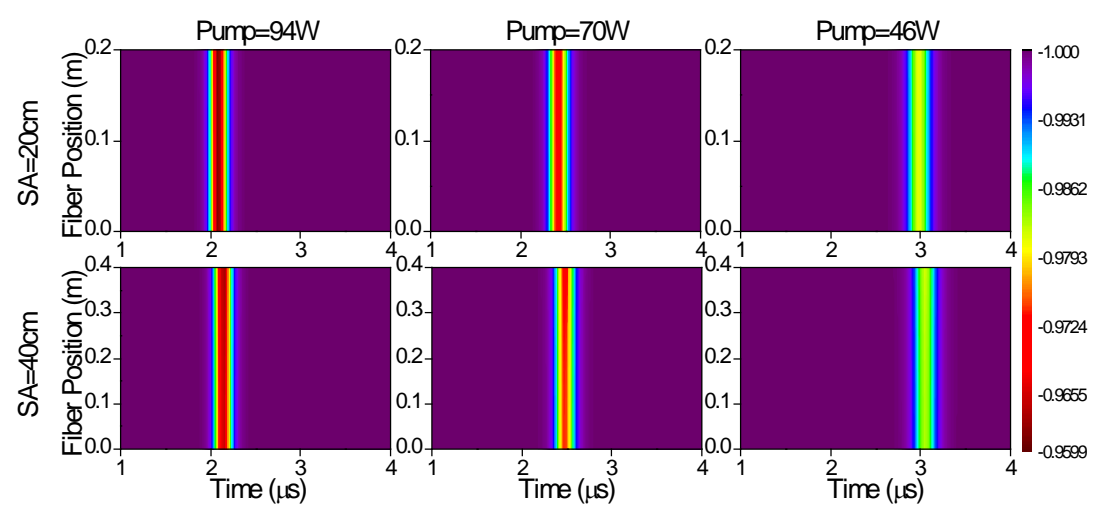

Fig. 2.11 Inversion distribution in saturable absorber fiber under different pump amplitude.

The study of dynamic in the fiber laser cavity above provides insight for laser characteristic. The pump amplitude is important to improve the peak power and pulse duration. However, the available pump amplitude for laser is always limited in real applications. The laser must be optimized for higher peak power and better efficiency for given pump amplitude.

\subsection{Optimization and discussion}

The laser performance in terms of peak power, pulse duration, and pulse energy is affected by many parameters, including but not limited to fiber length, dopant concentration, and core diameter of gain fiber as well as of SA fiber, lasing wavelength, and output coupler reflectivity. It is hardly possible to calculate all laser performance in such multi-dimensional parameter space. In order to simplify the related discussion, we use passively Q-switched laser with $50 \mathrm{~cm}$ SA fiber 
length as basis, perform several simulations by changing one parameter a time and fixing all others, and illustrate the basic relation and influence of each parameter.

The fiber length and dopant concentration (pump absorption ratio) of gain fiber will be studied first. Peak power, pulse duration, pulse energy for the laser with different gain fiber length, pump absorption ratio, and pump amplitude is compared in Fig 2.12. When the fiber absorption at pump wavelength is $5.4 \mathrm{~dB} / \mathrm{m}$ (which used in the experiment), the optimal length of gain fiber for obtaining highest peak power is at about $5 \mathrm{~m}$. The peak power increases about $8 \%$ per meter increased in gain fiber length until $5 \mathrm{~m}$. Then the peak power starts to degrade with further increment of gain fiber length. We choose $4.5 \mathrm{~m}$ of gain fiber in the experiment, which is about the optimal for the generation of highest peak power.

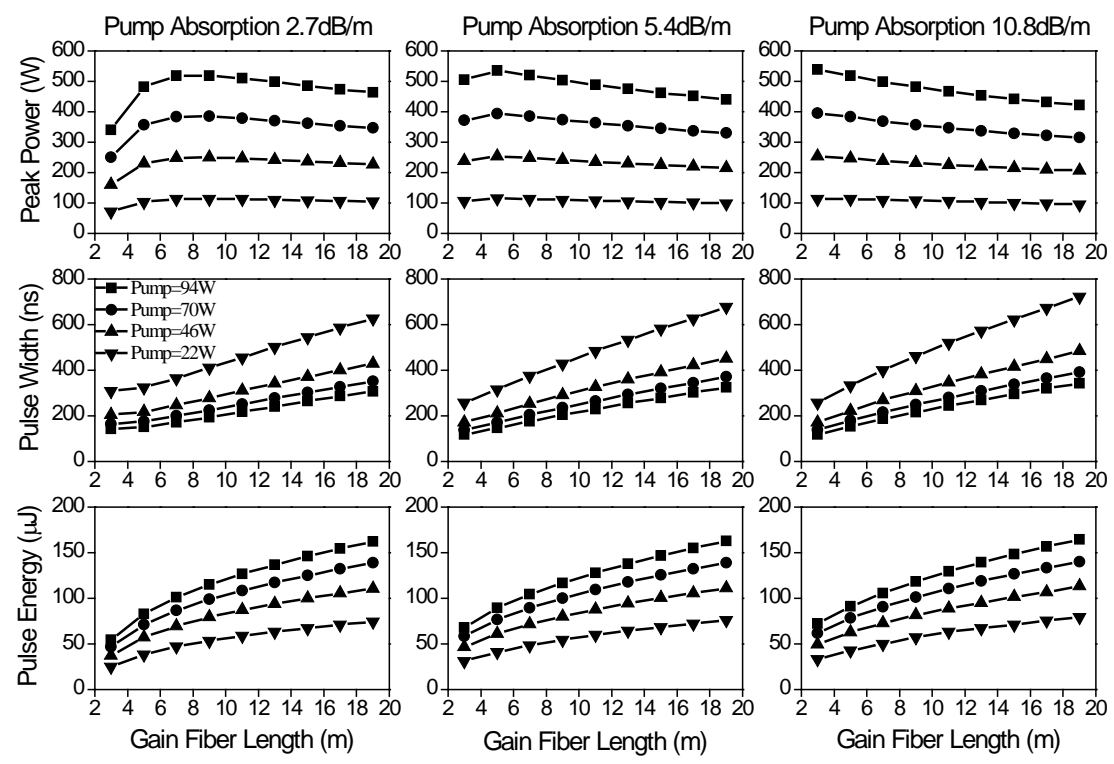

Fig. 2.12 Peak power, pulse width, and pulse energy for laser with different gain fiber length, pump absorption ratio, and pump amplitude.

Increasing gain fiber length elevates total pump absorption and gain ratio of the laser, thus larger pulse energy is expected. However, the pulse duration is also increased, so the overall effect on peak power converges to an optimal point. Also, by increasing the dopant concentration or pump absorption, thus increasing 
the pump absorption rate, we see the optimal length shifts to shorter value, indicating that the dopant concentration plays a scaling factor for the fiber length. Notably, increasing dopant concentration also reduces the pulse duration for same length of gain fiber when the dopant concentration is low. Therefore, high dopant concentration is always preferred. However, when the dopant concentration is too high, non-linear effect such as ion-pair clustering appears, which leads to losses both pump power and signal in lasers operating $[19,20]$.
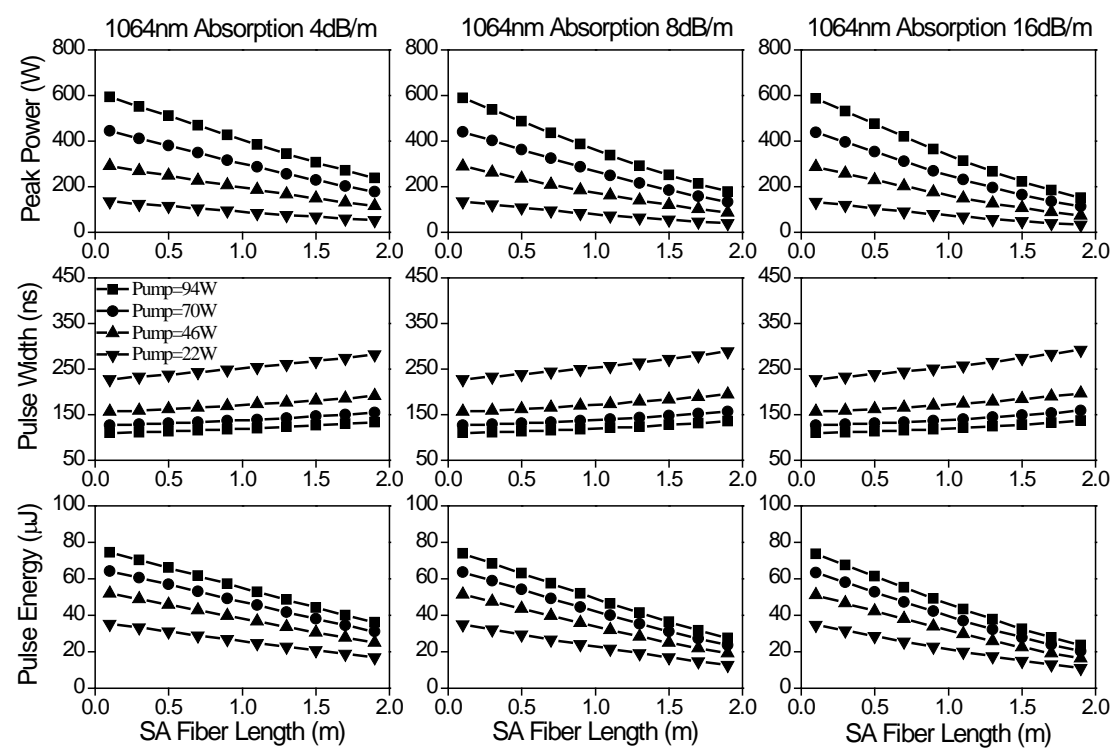

Fig. 2.13 Peak power, pulse width, and pulse energy for laser with different SA fiber length, 1064nm absorption ratio, and pump amplitude.

We perform similar analysis for SA fiber. In Fig 2.13, peak power, pulse duration, pulse energy for the laser with different fiber length, dopant concentration, and pump amplitude is compared. Simulation suggested that to obtain higher peak power, shorter absorber fiber length is preferred. As absorber fiber length increases, pulse energy decreases. Recall in the previous section in Fig 2.9, increase absorber fiber length indeed brings the population inversion in active fiber to a higher level. However, the ASE level required to onset the Q-switch is also elevated, leads to merely improvement in overall energy extraction in the Qswitching process. Plus the loss in saturable absorber is also increased, resulting degradation in pulse energy. Increase in dopant concentration in absorber fiber 
leads the decrease in peak power and pulse energy more significant when increase absorber fiber length, and thus, not preferred. Pulse duration is not essentially affected by either length, or dopant concentration of SA fiber.

Next, we study the effect of core diameter ratio of gain fiber to absorber fiber. The laser performance with different core diameter of gain fiber and absorber fiber, and pump amplitude is compared in Fig 2.14. The gain fiber length and SA fiber length is set back to $4.5 \mathrm{~m}$ and $0.5 \mathrm{~m}$, respectively. We assume constant dopant concentration, while changing the core diameter of the gain fiber. Thus, the pump absorption ratio increases as core diameter. The pulse duration increases significantly with the increment of the core diameter of gain fiber, and is not affected by decrement of the core diameter of absorber fiber. The pulse energy improves linearly and significantly as the gain fiber core diameter increases. However, pulse energy improves slightly with decrement of absorber fiber core diameter. It indicates excessive pump absorption leads increasing in pulse duration.
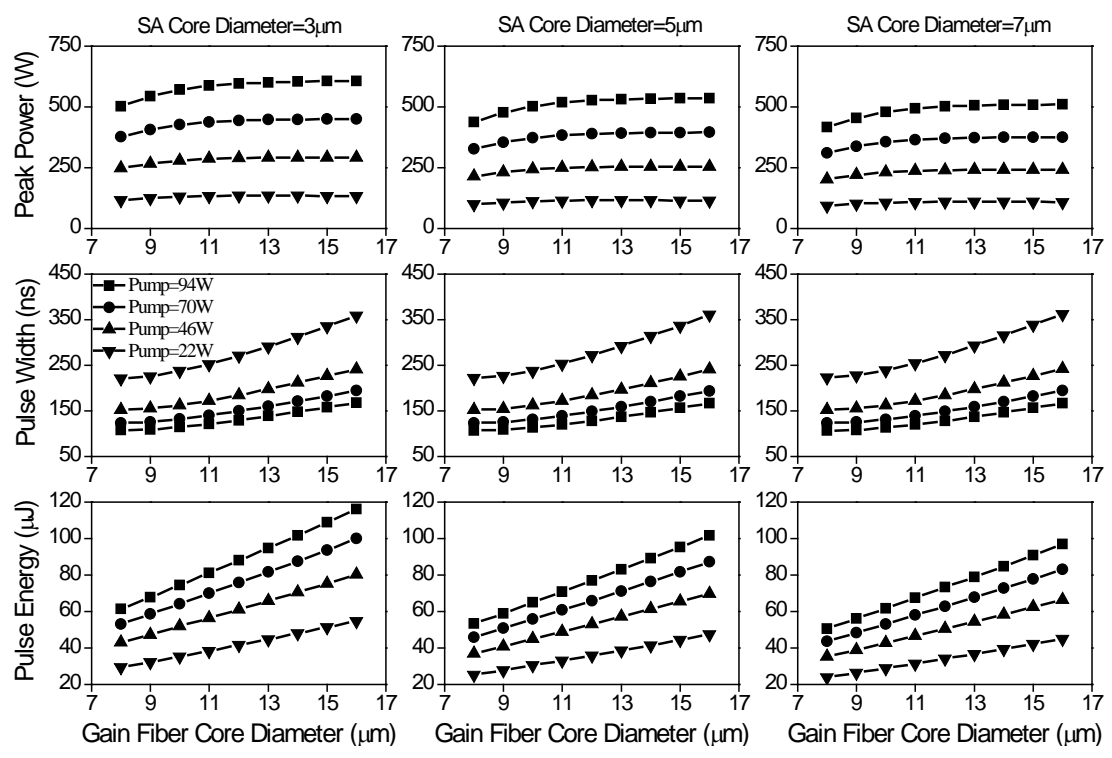

Fig. 2.14 Peak power, pulse width, and pulse energy for laser with different gain fiber core diameter, absorber fiber core diameter, and pump amplitude. 
Last, we consider the laser performance as a function of output coupler reflectivity and laser wavelength in Fig 2.15. We noticed that by decrease the output coupler reflectivity by $5 \%$, the peak power is improved by $10 \%$. The pulse energy is also increased by $10 \%$, and the pulse duration is not affected. As increasing the laser wavelength, both pulse duration and pulse energy are increasing simultaneously.

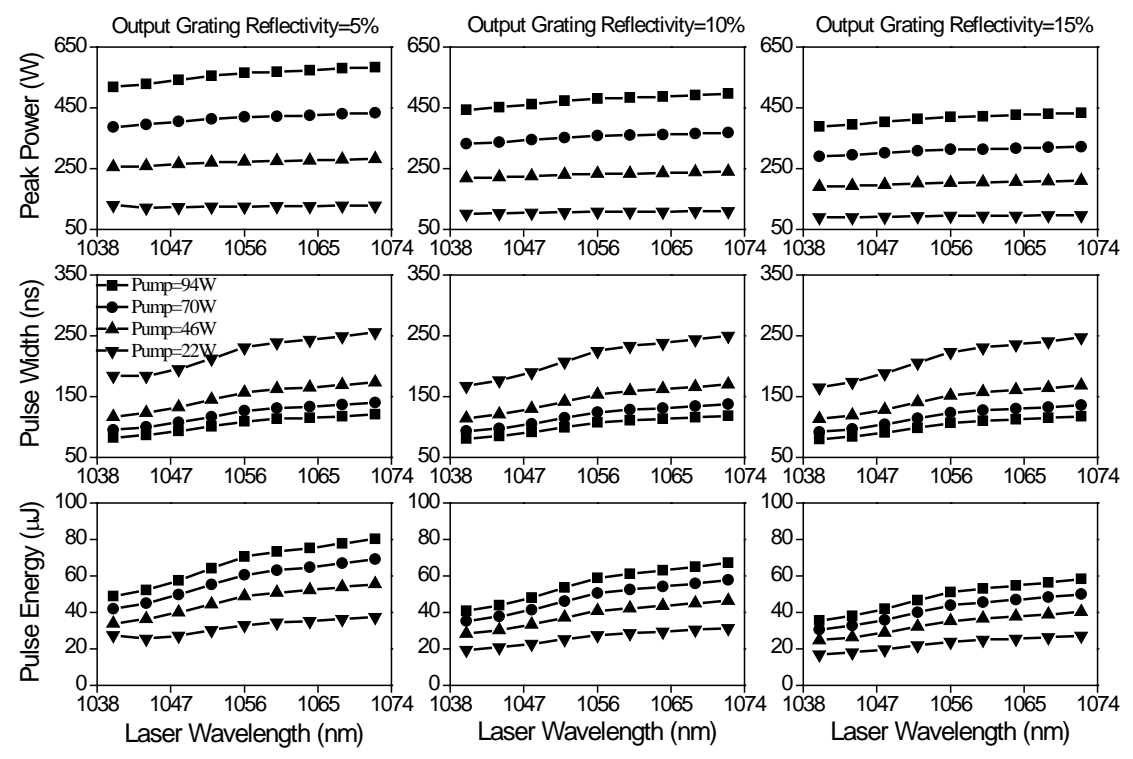

Fig. 2.15 Peak power, pulse width, and pulse energy for laser with different laser wavelength, output coupler reflectivity, and pump amplitude.

Since the absorption cross section of $\mathrm{Sm}$ is increasing monotonically from $1040 \mathrm{~nm}$ to $1070 \mathrm{~nm}$, the unsaturated loss in absorber fiber is increased, thus, more energy is extracted from gain fiber, producing larger pulse energy. However, the emission cross section of $\mathrm{Yb}$ decreases as shifting to longer wavelength, degrading the extraction rate, thus, longer pulse duration. The overall effect on pulse peak power is slightly positive when the lasing wavelength is shifted to longer wavelength. We notice that in all these studies, adjustment of the pump amplitude will only change slightly the shape of the function for pulse width and pulse energy. However, peak power increases linearly as increment of pump amplitude when other parameters are fixed. 


\section{$2.7 \quad$ Summary}

Designs using all-fiber configuration have advantages in flexibility and portability compare to the bulk design. The repetition rate of the presented approach is controlled by the frequency of the power source that drives the laser diodes, thus, enable a truly repetition rate controllable pulsed laser. Stable pulses with energy of $68 \mu \mathrm{J}$, duration of $210 \mathrm{~ns}$ are obtained experimentally. The average power is measured as $6.8 \mathrm{~W}$ at a repetition rate of $100 \mathrm{kHz}$ and the peak power is calculated at $324 \mathrm{~W}$. Mode area mismatch technique is incorporated in the design and relative high energy is produced with Sm-doped SA fiber. A theoretical model is developed with proper consideration of multi-wavelength nature of ASE, which predicts accurate laser characteristics, such as pulse energy and peak power. Optimization of all-fiber passively Q-switched Yb:Sm fiber laser has also been presented.

Dynamic of pulse buildup mechanism is extensively analyzed, and laser performance versus several possible laser design parameters is studied. Laser output characteristics, including peak power, pulse energy, pulse duration, are determined by the evolutions of inversion distribution and ASE buildup, which depend on fiber length, dopant concentration, core diameter, output coupler reflectivity, wavelength, and pump amplitude. High dopant concentration in gain fiber is preferred as it is a scaling factor for short fiber length, and thus shortens pulse duration. Short fiber length and moderate dopant concentration is favored for the SA fiber to obtain high peak power. The peak power to pump amplitude ratio is kept as constant for moderate pump level, indicating high pump power is preferred. Instead of using amplification stage, more diodes could be combined through a power combiner to obtain higher laser pulse energy and peak power. Also, this design offers a variable repetition rate controlled by the modulation of pump which will find many applications in industrial and medical fields. 


\section{Chapter 3}

\section{All-fiber passively Q-switched fiber laser with $\mathbf{Y b -}$}

\section{doped fiber saturable absorber}

\subsection{Introduction}

When selecting a proper SA fiber for Q-switched laser, large absorption coefficient for the desired wavelength band is preferred, since a quickly saturating SA results in pulses of short duration due to the large unsaturated gain while the SA is bleached. Mode field mismatching is proposed in [13] to address this problem by creating a large photon density ratio to overcome the cross-section ratio. However, Even if the adiabatically tapered splicing is available, the coupling loss between the mode-mismatched fibers is still unavoidable high and limits the design.

One additional way has been suggested in [10], where the SA, Bi-doped fiber, is placed in a separate cavity to achieve laser action of its wavelength and consequently decrease the recovery time from excited state. Pulse train with pulse duration of 1 ss was successfully produced. The same technique has been incorporated in [21], where Yb-doped gain fiber coupled with Ho-doped SA fiber, and produced $70 \mathrm{~J} 250 \mathrm{~ns}$ pulse, but with rather poor stability.

Soh et al. proposed an all-fiber configuration for a passively Q-switched laser, in which, both gain fiber and SA fiber were Yb-doped, however, with a large core size difference [22]. Their simulation showed that $0.5 \mathrm{~mJ}$ pulse energy and $14 \mathrm{~ns}$ pulse width could be realized. Very recently, the same group successfully verified this configuration and reported Q-switched oscillator with $40 \mu \mathrm{J}$ and 79 ns pulses 
at $1026 \mathrm{~nm}$ [15]. However, the laser used bulk lenses to couple the pump laser into both gain fiber and SA fiber and dichroic mirrors to select wavelengths.

A truly all-fiber configuration for passively Q-switched all-fiber laser is presented here. Both mode field mismatch and an additional cavity were employed for SA in our design. The laser produces highly stable pulses with $86 \mu \mathrm{J}$ pulse energy and 232 ns pulse duration. Theoretical model is modified to include the additional cavity enclosing SA fiber. The effects of design parameters on laser performance, such as pulse energy, pulse duration, and peak power is also studied.

\subsection{Experiment setup}

In this experiment, we used a $(3+1)$ x1 power combiner from ITF Lab to couple power provided from 3 pump diodes, each rated at 25W with wavelength at 975 $\mathrm{nm}$, to the Yb-DCF. The schematic diagram is shown in Fig 3.1. The signal port on the pump side of the combiner enables backward pumping scheme, where the laser power is in opposite direction of the pumping power in the linear cavity. The combiner has insertion loss of $0.03 \mathrm{~dB}$ between signal to output port, and $0.4 \mathrm{~dB}$ between pump to output port. The output port of the combiner is 10/125 DCF, tapering spliced to the 15/130 Yb-DCF active fiber. The active fiber has nominal absorption rate of $5.4 \mathrm{~dB} / \mathrm{m}$ at $976 \mathrm{~nm}$. The length of the active fiber is $4.5 \mathrm{~m}$.

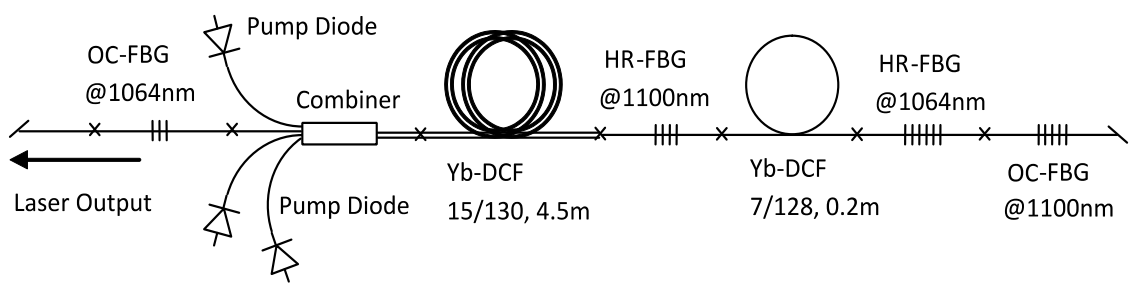

Fig. 3.1 Schematic diagram of all-fiber passively Q-switched fiber laser with Yb-DCF as saturable absorber.

The absorber fiber is 7/128 Yb-DCF, which has estimated core absorption rate of $4 \mathrm{~dB} / \mathrm{m}$ at $1064 \mathrm{~nm}$. The absorber fiber of $20 \mathrm{~cm}$ long is enclosed by a pair of gratings at $1100 \mathrm{~nm}$, of which, the strong grating, spliced between the gain fiber and the absorber fiber, has reflectivity of $35 \mathrm{~dB}$ (99.9\%), while the other one has 
reflectivity of $13 \mathrm{~dB}$ (95\%). This cavity at $1100 \mathrm{~nm}$ around the absorber fiber reduces the recovery time from saturation, as suggested in [22]. Please note that the ratio of the core area between active fiber and absorber fiber is 4.6. Such a high ratio in mode field area shall result in rapid saturation and high extraction efficiency. The gain fiber is adiabatically tapered to match the mode field diameter of the small-core saturable absorber.

The output coupler grating at $1064 \mathrm{~nm}$ is $4 \%$ in reflectivity. The two fiber ends are spliced to their matching angle polished fiber connectors (FC/APC) in order to prevent reflection. The pump striper is applied between active fiber and HR-FBG @1100nm to prevent pump power entering the SA fiber. Finally, in order to attenuating the output for pulse trace and spectrum measurement, a lens of $62 \mathrm{~mm}$ focal length is used to couple the output to a patch cord, which could be freely inserted to either a photodiode or an optical spectral analyzer.

\subsection{Results and discussion}

The three diodes were connected in series and driven by pulsed power supply, which has adjustable duty cycle and frequency up to $100 \mathrm{kHz}$. The laser was tested under three frequency rate, 10, 60, and $100 \mathrm{kHz}$. For each frequency, the measurement was recorded for different current amperage from $5 \mathrm{~A}$ to $11 \mathrm{~A}$, which was $28 \mathrm{~W}$ to $60 \mathrm{~W}$ of estimated pump power. The duty cycle was adjusted for each operation condition so that no occurrences of after-pulse in pulse train.
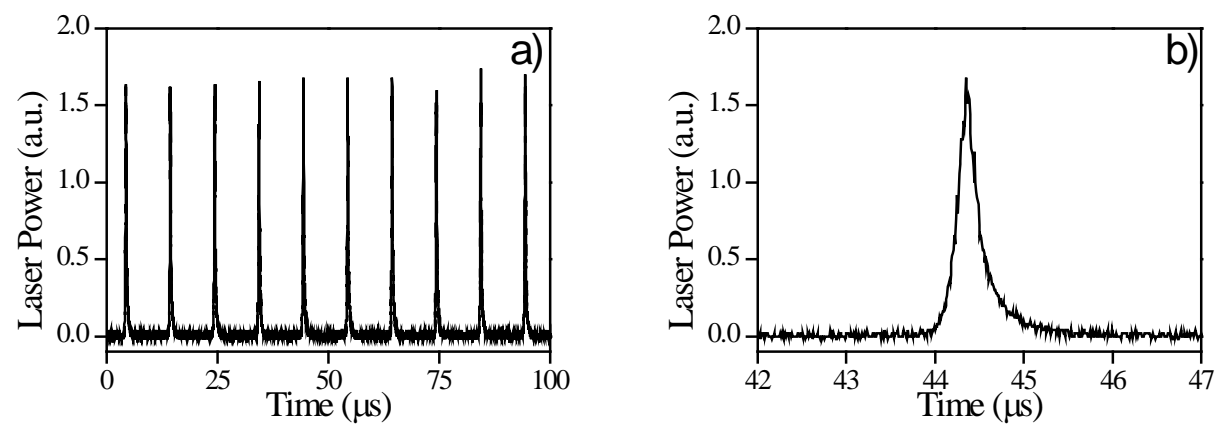

Fig. 3.2 a) Oscilloscope trace of the Q-switching pulses at $100 \mathrm{kHz}$ rate; and b) a single pulse with $86 \mu \mathrm{J}$ energy and $232 \mathrm{~ns}$ (FWHM) pulse width. 
Steady pulse trains were achieved in all conditions above. At $100 \mathrm{kHz}$ frequency and $60 \mathrm{~W}$ of pump power, train of pulses with $232 \mathrm{~ns}$ pulse width and $86 \mu \mathrm{J}$ pulse energy was observed. The peak power was estimated at $371 \mathrm{~W}$, as shown in Fig 3.2. The pulse amplitude variation was calculated to $4 \%$ standard deviation and the pulse duration varied about $1.8 \%$. Similar pulse amplitude stability and pulse jitter were observed at other operation conditions with different pump power and pump frequency. Fig 3.3.shows two more examples of stable pulse train in other repetition rates. The low pulse amplitude variation is also an evident, which indicates that the SA is well recovered between pulses.
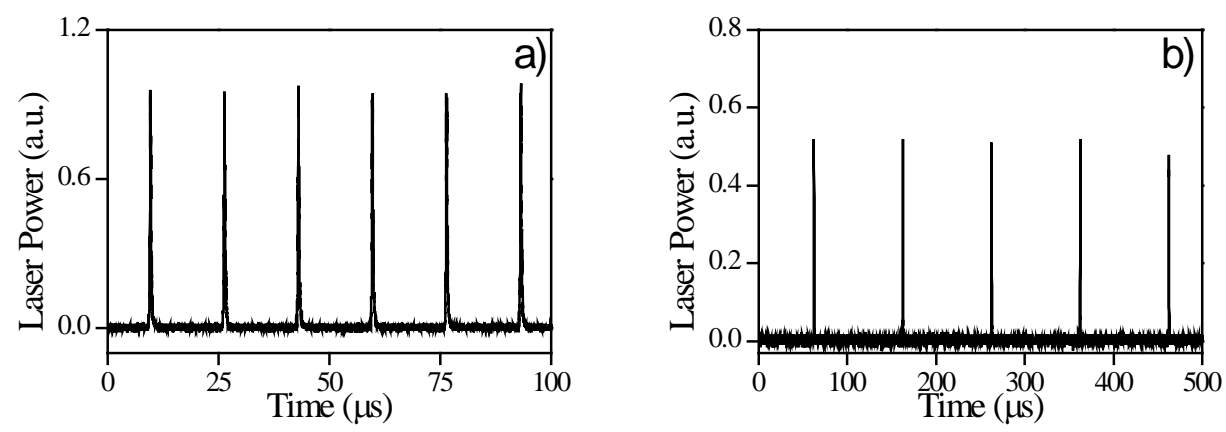

Fig. 3.3 Oscilloscope traces of the Q-switching pulses at a) $60 \mathrm{kHz}$, and b) $10 \mathrm{kHz}$.

The laser spectrum is measured by the ANDO AQ6317 optical spectrum analyzer. Narrow line width of $0.164 \mathrm{~nm}$ at $1064 \mathrm{~nm}$ was observed at $60 \mathrm{~W}$ of pump amplitude. Narrower line width was also obtained at lower pump amplitude, as shown in Fig 3.4. No significant laser power at $1110 \mathrm{~nm}$ (the inner cavity enclosing SA) was observed. It indicates this design schema, which reduces SA recovery time, merely introduce unexpected lasing wavelength.

Even when the pump amplitude reached as high as $60 \mathrm{~W}$, there was no significant non-linear effect, such as stimulated Brillouin scattering (SBS) and stimulated Raman scattering (SRS). SRS is the relevant limit in this case and the limiting peak power is estimated as $2.9 \mathrm{~kW}$, by using the equations in [23]. The current peak power, $371 \mathrm{~W}$, is far less than the limitation. Thus, this laser system is promising to generate higher peak power, when adding more pump power. 

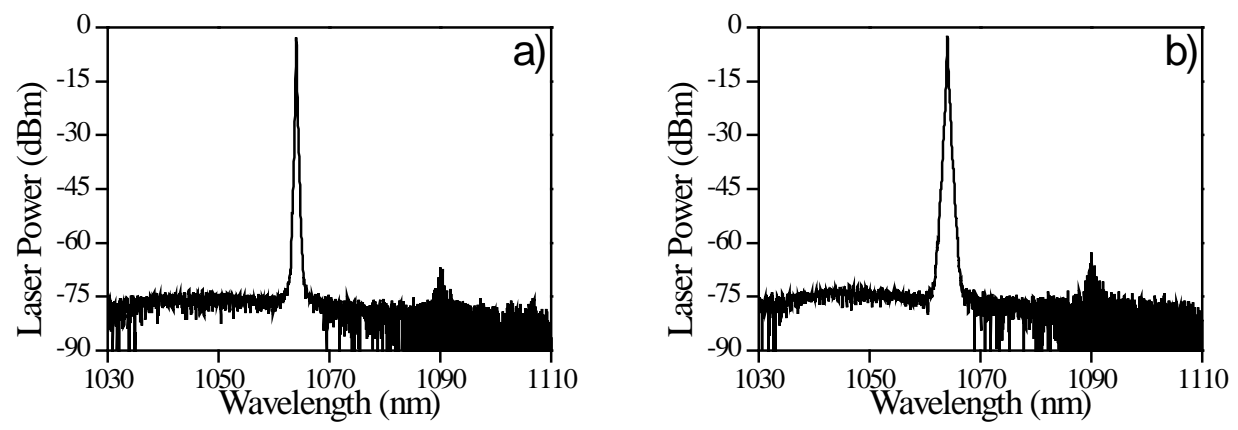

Fig. 3.4 Emission spectrum of the laser at a) $28 \mathrm{~W}$ of power amplitude (line width is 113 pm), and b) $60 \mathrm{~W}$ of power amplitude (line width is $164 \mathrm{pm}$ ).

Although the spectral line width is broadening with increasing pump amplitude, the factor is insignificant. As shown in Fig 3.5(a), the slope is estimated at 1.81 pm per $1 \mathrm{~W}$ increase at pump amplitude. For applications as metal cutting and most material processing which is not sensitive to the laser spectral, this laser shows potential to scale up power to kilowatts level.
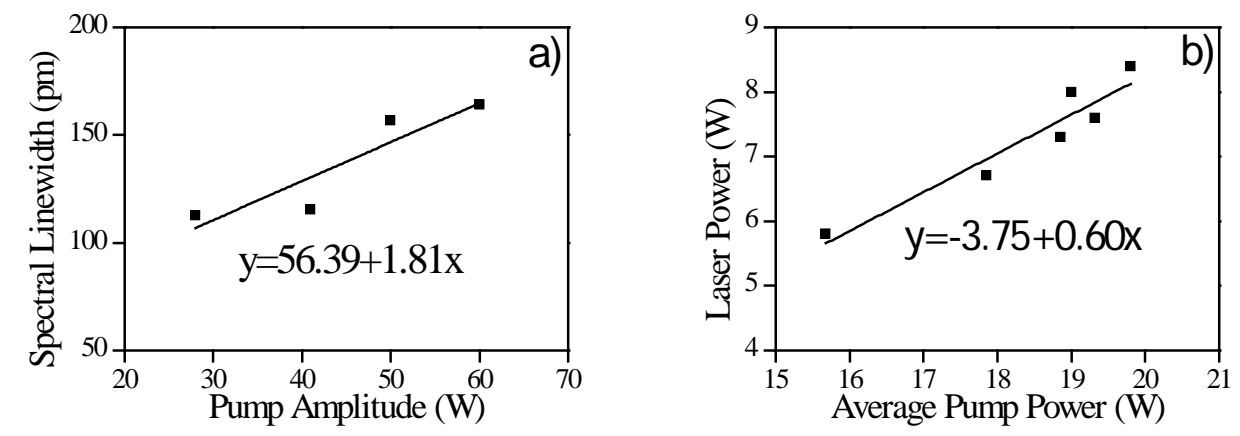

Fig. 3.5 a) Spectral line width versus pump power amplitude; and

b) Output laser power versus average pump power.

At $100 \mathrm{kHz}$, the average pump power is calculated by multiplying pump amplitude with modulated pump duty cycle, and is plotted against the measured laser output power in Fig 3.5(b). Slope efficiency of $60 \%$ is achieved for this configuration. The duty cycle is set to $33 \%$ at $60 \mathrm{~W}$ pump amplitude, which is 3.3 $\mu \mathrm{s}$. The remaining time in a cycle is only $6.7 \mu \mathrm{s}$. We know that the Yb has lifetime of $840 \mu$ s, which is a factor of more than 100 times. Because no higher peak power was observed in the lower frequency regime, such as $10 \mathrm{kHz}$, the SA 
is well recovered in the extreme short period of time compare to its lifetime. Therefore, it indicates the $1100 \mathrm{~nm}$ cavity around SA significantly reduces the recovery time of SA. Due to limitation of the power supply, no higher frequency is available to continue experiments to accurately estimate the recovery time.
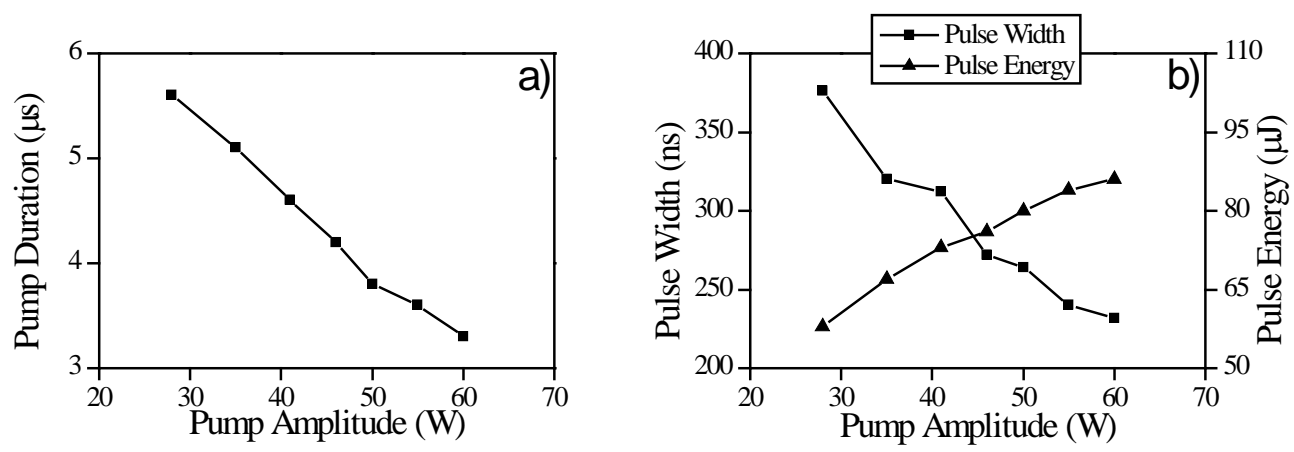

Fig. 3.7 a) Pump duration versus pump amplitude; and

b) laser pulse width and pulse energy versus pump power amplitude.

We measured the pump duration as function of pump power amplitude at pump modulation frequency of $100 \mathrm{kHz}$. The pump duration is fine adjusted for each pump amplitude to avoid the occurrence of the after pulses. In Fig 3.6(a), as the pump amplitude increases from $28 \mathrm{~W}$ to $60 \mathrm{~W}$, the pump duration is reduced from $5.6 \mu$ s to $3.3 \mu$ s. The corresponding laser pulse width and pulse energy were plotted in Fig 3.6(b). The pulse width decreases from 376 ns to 232 ns, and the pulse energy increases from $58 \mu \mathrm{J}$ to $86 \mu \mathrm{J}$. Higher pump amplitude leads to higher gain in the active fiber and thus takes less time depleting the population inversion after the on-set of the lasing. Therefore, higher pulse energy with narrower pulse width will be generated with higher pump amplitude. The pulse energy curve shows no saturation indicates high scalability for the pulse energy. However, the pulse width shows rolled over at around $70 \mathrm{~W}$ of pump amplitude. The factor that limiting the pulse width to go further short is under study.

\subsection{Numerical model and optimization}

We use the theoretical model developed in chapter 2 and modify several equations to include additional features, specific for this configuration. Since the backward 
pumping scheme is used in this configuration, the pump direction is opposite to the output. We change the pump power denote to $P_{p}^{-}$in all equations. The governing rate equations for gain fiber remain the same otherwise.

The pump power has been stripped before entering the SA fiber, thus spontaneous emission is omitted in SA fiber. However, ASE generated from gain fiber is entering the SA fiber and the enclosing secondary cavity is placed to induce lasing of SA fiber. Therefore, both absorption cross-section and emission crosssection must be considered. The modified equations for SA fiber are shown below:

$$
\begin{aligned}
& N_{0}^{s a}=N_{1}^{s a}(x, t)+N_{2}^{s a}(x, t) \\
& \frac{\partial N_{2}^{s a}(x, t)}{\partial t}=\sum_{k} \frac{\Gamma_{k}^{s a} \lambda_{k}}{h c A_{c a}^{s a}}\left[\sigma_{a k}^{s a} N_{1}^{s a}(x, t)-\sigma_{e k}^{s a} N_{2}^{s a}(x, t)\right]\left[P_{k}^{+}(x, t)+P_{k}^{-}(x, t)\right] \\
& -\frac{N_{2}^{s a}(x, t)}{\tau_{s a}} \\
& \frac{d P_{k}^{ \pm}(x, t)}{d x}= \pm \frac{\partial P_{k}^{ \pm}(x, t)}{\partial x}+\frac{1}{v_{k}^{s a}} \frac{\partial P_{k}^{ \pm}(x, t)}{\partial t} \\
& =\Gamma_{k}^{s a}\left[-\sigma_{a k}^{s a} N_{1}(x, t)+\sigma_{e k}^{s a} N_{2}(x, t)\right] P_{k}^{ \pm}(x, t)-\alpha_{k}^{s a} P_{k}^{ \pm}(x, t)
\end{aligned}
$$

The appearance of the secondary cavity is included in the boundary conditions. The length of all fiber gratings and power combiner are neglected for simplicity. We use $R_{2 H R}$ and $R_{20 C}$ to denote the reflectivity of HR-FBG and OC-FBG of secondary cavity, respectively. The modified boundary conditions are listed below:

$$
\begin{aligned}
& P_{p}^{-}(L, t)=P_{\text {pump }}(t) \\
& P_{k}^{-}(L, t)=R_{o c} P_{k}^{+}(L, t) \\
& P_{k}^{+}\left(0^{+}, t\right)=(1-\eta) P_{k}^{+}\left(0^{-}, t\right)+R_{2 H R} P_{k}^{-}\left(0^{+}, t\right) \\
& P_{k}^{+}\left(-L_{s a}, t\right)=\left(R_{H R}+R_{2 O C}\right) P_{k}^{-}\left(-L_{s a}, t\right)
\end{aligned}
$$




$$
P_{k}^{-}\left(0^{-}, t\right)=(1-\eta) P_{k}^{-}\left(0^{+}, t\right)+R_{2 H R} P_{k}^{+}\left(0^{-}, t\right)
$$

where $\eta$ is the sum of splice loss between gain fiber and secondary HR-FBG and SA fiber. Note that since the response band of main cavity HR-FBG and secondary cavity OC-FBG are well separated by more than $30 \mathrm{~nm}$, simple matrix summation between the two reflectivity is sufficient.

The gain fiber used in the experiment is $\mathrm{Yb}$-doped double cladding fiber from Nufern (LMA-YDF-15/130-VIII). The nominal cladding absorption coefficient is $5.4 \mathrm{~dB} / \mathrm{m}$ near $975 \mathrm{~nm}$. The dopant concentration is estimated to be $2.14 \times 10^{25} \mathrm{~m}^{-}$ ${ }^{3}$. The SA fiber is from CorActive (DCF-YB-7/128-FA). Although it is double cladding fiber, since the pump is stripped off before entering it, all interaction take place only in core. The dopant concentration is estimated to be $1.45 \times 10^{25}$ $\mathrm{m}^{-3}$. The rest parameters are shown in Table 3.1 as given in Ref [18]:

\begin{tabular}{|l|l|l|l|l|l|}
\hline Parameter & Value & Parameter & Value & Parameter & Value \\
\hline$\sigma_{e k}$ & $0.35 \times 10^{-24} \mathrm{~m}^{2}$ & $N_{0}$ & $2.14 \times 10^{25} \mathrm{~m}^{-3}$ & $\alpha_{k}$ (for all k) & $0.005 \mathrm{~m}^{-1}$ \\
\hline$\sigma_{a k}$ & $0.01 \times 10^{-24} \mathrm{~m}^{2}$ & $N_{0}^{s a}$ & $9.35 \times 10^{25} \mathrm{~m}^{-3}$ & $\alpha_{k}^{\text {sa } \text { (for all k) }}$ & $0.005 \mathrm{~m}^{-1}$ \\
\hline$\sigma_{e k}^{s a}$ & $0.35 \times 10^{-24} \mathrm{~m}^{2}$ & $\tau$ & $0.84 \times 10^{-3} \mathrm{~s}$ & $\alpha_{p}$ & $0.005 \mathrm{~m}^{2}$ \\
\hline$\sigma_{a k}^{s a}$ & $0.01 \times 10^{-24} \mathrm{~m}^{2}$ & $\tau_{s a}$ & $0.84 \times 10^{-3} \mathrm{~s}$ & $R_{O C}$ & $4 \%$ \\
\hline$\sigma_{a p}(\mathrm{p}=975 \mathrm{~nm})$ & $2.5 \times 10^{-24} \mathrm{~m}^{2}$ & $d_{c o r e}$ & $15 \times 10^{-6} \mathrm{~m}$ & $R_{H R}$ & $99.9 \%$ \\
\hline$\sigma_{e p}(\mathrm{p}=975 \mathrm{~nm})$ & $2.44 \times 10^{-24} \mathrm{~m}^{2}$ & $d_{c o r e}^{\text {sa }}$ & $6 \times 10^{-6} \mathrm{~m}$ & $R_{20 C}$ & $95 \%$ \\
\hline $\mathrm{NA}$ & 0.08 & $\mathrm{~L}$ & $4.5 \mathrm{~m}$ & $R_{2 H R}$ & $99.9 \%$ \\
\hline $\mathrm{NA}_{\mathrm{sa}}$ & 0.19 & Lsa & $0.3 \mathrm{~m}$ & $\eta$ & 0.5 \\
\hline
\end{tabular}

Table 3.1 Simulation parameters in passively Q-switched fiber laser with Yb-doped SA.

In the simulation, the pump is modulated at fixed frequency of $100 \mathrm{kHz}$. The duty cycle is controlled by program to turn-off precisely at the output peak. This technique is important to prevent the on-set of after-pulses. In the first set of simulation, we change the active fiber length from $3 \mathrm{~m}$ to $7 \mathrm{~m}$, SA fiber length from $0.1 \mathrm{~m}$ to $1 \mathrm{~m}$, and pump amplitude from $46 \mathrm{~W}$ to $94 \mathrm{~W}$. The results are organized and plotted in Fig 3.8. For fixed active fiber length, the pulse width 
increases as the increase of SA fiber length at rate of 9\% per meter when pumping at $70 \mathrm{~W}$ pump amplitude. The rate slightly increases for lower pump amplitude.
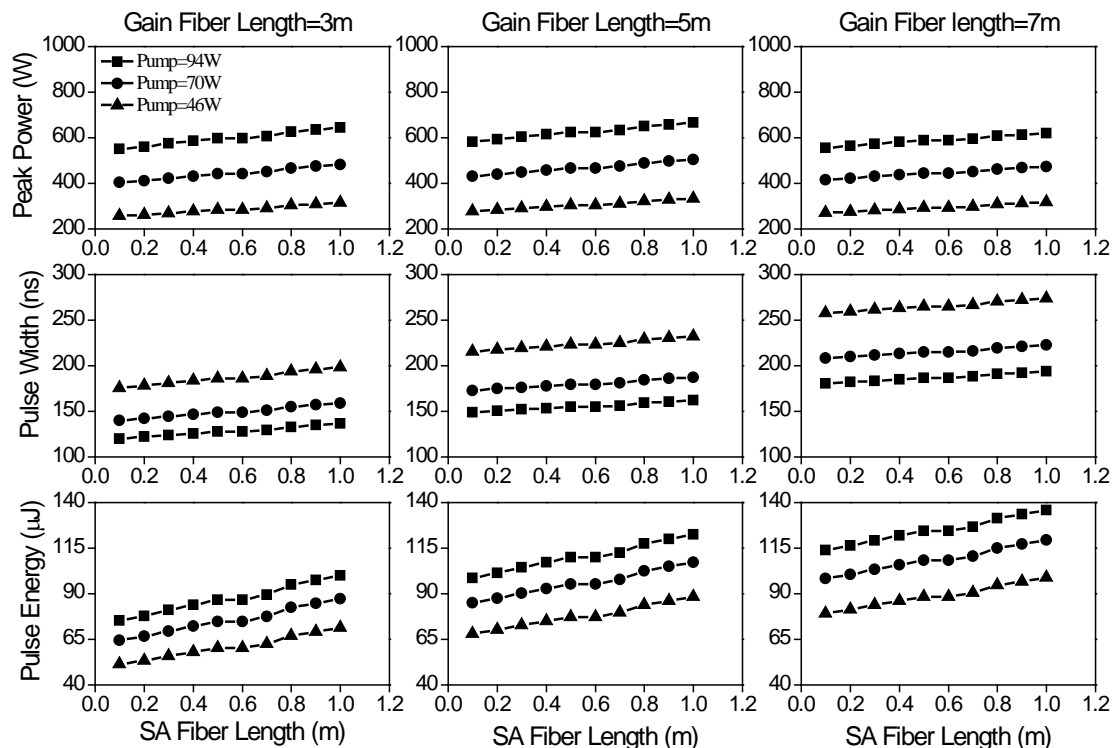

Fig. 3.8 Peak power, pulse width, and pulse energy of $\mathrm{Yb}$ doped fiber laser with different active fiber length, SA fiber length, and pump amplitude.

The pulse energy increases with SA fiber length as well and the slope is about $26 \%$ per meter, which is far higher than the increase rate of pulse width. As the result, the peak power increases at about $18 \%$ per meter. Long SA fiber elevates the ASE threshold and increases the population inversion in the gain fiber to produce high pulse energy. However, when the length of cavity is too long, non-linear effect might be induced and degrade laser performance. The increase of active fiber also increases the pulse width and pulse energy. However, it shows roll-off at around $5 \mathrm{~m}$ for peak power, indicating the optimize value. The lengthy nature of fiber laser is a limiting factor for pulse width. Short cavity provides insufficient pump absorption and is not preferred. 

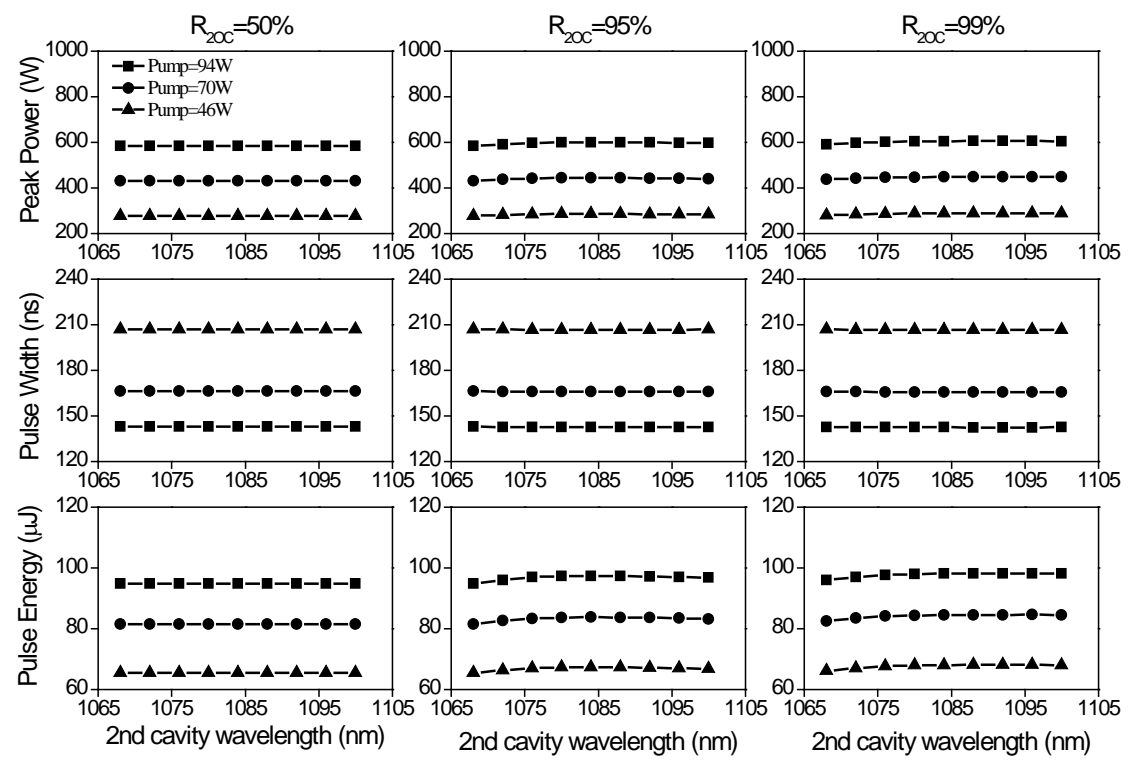

Fig. 3.9 Peak power, pulse width, and pulse energy of $\mathrm{Yb}$ doped fiber laser with different secondary gratings wavelength, OC reflectivity, and pump amplitude.

The effect of secondary cavity enclosing the SA fiber is shown in Fig 3.9. In this set of simulation, the active fiber length is set to $4.5 \mathrm{~m}$ and the SA fiber length is set to $0.3 \mathrm{~m}$. When the secondary OC-FBG increases from $50 \%$ to $99 \%$, the result peak power increases around $2 \%$ for cavity wavelength at $1100 \mathrm{~nm}$. The effect is weaker for shorter wavelength. Pulse width decreases for longer cavity wavelength, and the effect is stronger for higher secondary OC-FBG reflectivity. Pulse energy increases for longer cavity wavelength, and leads to increases of peak power for longer cavity wavelength. Thus, secondary cavity wavelength at $1100 \mathrm{~nm}$ is preferred in design.

\subsection{Summary}

A passively Q-switched Yb-doped double cladding fiber laser is realized by coupling with mode field mismatched Yb-doped SA fibers. High mode field ratio of 4.6 is achieved to produce pulses with $86 \mu \mathrm{J}$ pulse energy and 232 ns pulse duration. The peak power is estimated to be $371 \mathrm{~W}$ without amplification stage. A secondary cavity of $1100 \mathrm{~nm}$ to enclose the SA fiber has also been employed in the design. The laser produces stable pulses without non-linear effect. Such a 
reliable laser can be further scale up for material processing and laser micromachining. The average output power of $8.6 \mathrm{~W}$ will make this laser useful for thin metal sheet cutting and other material processing. Travelling wave method has been used to develop the theoretical model and the effect from the secondary cavity has also been included. Laser performance characteristics in terms of pulse energy, pulse width, and peak power depend on gain fiber length, SA fiber length, secondary cavity wavelength, output coupler reflectivity and etc. Moderate length $\mathrm{Yb}$-doped SA fiber is preferred for producing high energy pulses, as it elevates population inversion in gain fiber and more energy might be extracted. 


\section{Chapter 4}

\section{All-fiber passively Q-switched fiber laser with}

\section{excessive long Yb-doped fiber}

\subsection{Introduction}

In every design of all-fiber Q-switched laser, saturable absorber is essential. Researchers have proposed several innovative configurations and techniques to induce the mechanism of saturating absorbing. The first all-fiber configuration uses Sm-doped fiber as SA to couple with Yb-doped fiber. Only long pulse duration is produced due to the ineffective absorption of the SA [7]. Recent researching shows it is possible to use SA, which the same doping rare-earth element. An Er-doped gain fiber coupling with an unpumped Er-doped fiber as SA produces $8 \mu \mathrm{J} 80 \mathrm{~ns}$ pulses of $1530 \mathrm{~nm}$ wavelength [11]. Passively Qswitching is also achieved in Yb-doped fiber laser [13], where uses an unpumped Yb-doped fiber as SA to produce $2.8 \mu \mathrm{J} 280 \mathrm{~ns}$ at $1064 \mathrm{~nm}$. Similar configuration is proposed by Soh et al. [15, 22]. In their experiment, pulses with $40 \mu \mathrm{J}$ pulse energy and 79 ns pulse duration have been produced.

In fiber laser system, when the gain fiber is extreme long, the far end of the gain fiber is insufficiently pumped and attenuating the signal power. The optimized fiber length is chosen to avoid such effect, so that the product with pump absorption coefficient is in the range of 15-18 dB [16]. We choose an excessive long Yb-doped fiber in our configuration. The insufficiently pumped section of the gain fiber providing the mechanism similar to SA and steady Q-switched pulse train is observed. The laser produces $82 \mu \mathrm{J}, 148 \mathrm{~ns}$ pulses. 
Theoretical model and simulations also support that the pump power is negligible at the end of the excessive long Yb-doped fiber. Population inversion at end of the fiber is mainly caused by the ASE, generated in the sufficiently pumped part of the gain fiber.

\subsection{Experiment setup}

We use a Yb-doped double cladding fiber as gain fiber, supplied by Nufern (LMA-YDF-10/130-VIII). It has nominal absorption coefficient of $3.9 \mathrm{~dB} / \mathrm{m}$ at $975 \mathrm{~nm}$. When we use $9 \mathrm{~m}$ long fiber, which provides $35 \mathrm{~dB}$ total absorption. Highest peak power of $554 \mathrm{~W}$ is achieved from this design. The generic configuration is shown in Fig 4.1. Three laser diodes, each rated at 25W, are spliced to the pump input port of a 7x1 multimode power combiner. The maximum insertion loss from the pump input ports to the output port is $0.3 \mathrm{~dB}$ (or $6.7 \%$ loss). Before assembly the rest of the laser system, the power at output port of the combiner is measured at different current level. This will be the basis of calculating the input power in the experiment. The usage of a combiner is to provide more input power to the laser cavity. If one pump diode is sufficient in the design, the combiner can definitely be omitted. The laser cavity is composed nothing more than a piece of Yb-doped double cladding fiber and a pair of FBGs. The high reflection grating (HR-FBG) is centered at near $1064 \mathrm{~nm}$ and has reflectivity of greater than 99\%. The matching output coupler grating (OC-FBG) has reflectivity of $6 \%$. Finally, an angle polished fiber connector (FC/APC) is spiced at the end to output the laser power. The FC/APC is 10/125, matching the fiber core/cladding size of the OC-FBG.

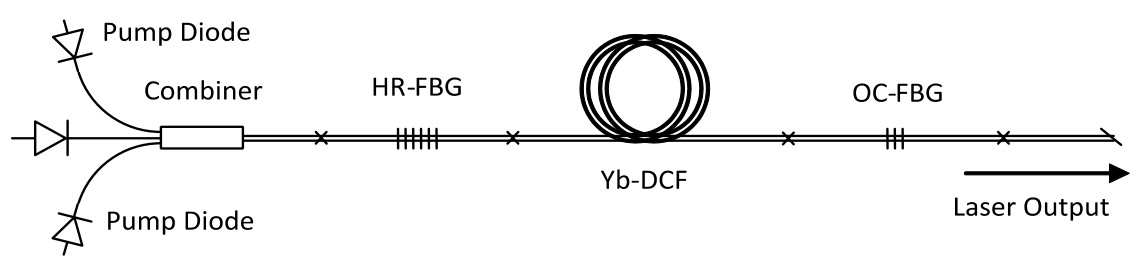

Fig. 4.1 Generic configuration of all-fiber passively Q-switched fiber laser with 9m long Yb-doped double cladding fiber. 
The laser output is imaged with a plano-convex lens to a patch cord to couple a few $\mathrm{mW}$ of power into the fiber for measurement, which is then inserted into either photodiode for oscilloscope trace or optical spectrum analyzer.

\subsection{Results and discussion}

The diodes are connected in series and triggered by a pulsed power supply. At each pump power level, we adjust the pump pulse duration, so that the after-pulse is completely compressed from the output. The laser is test under three pump repetition rates: $10 \mathrm{kHz}, 60 \mathrm{kHz}$, and $100 \mathrm{kHz}$. At repetition rate of $100 \mathrm{kHz}$, pulses of $82 \mu \mathrm{J}$ pulse energy and 148 ns pulse width (FWHM) were obtained. Fig. 4.2(a) shows the steady pulse train at repetition rate of $100 \mathrm{kHz}$. The pulse has a steep raising edge and a much steady falling tail as shown in Fig. 4.2(b), which is conformance to Q-switching theory.
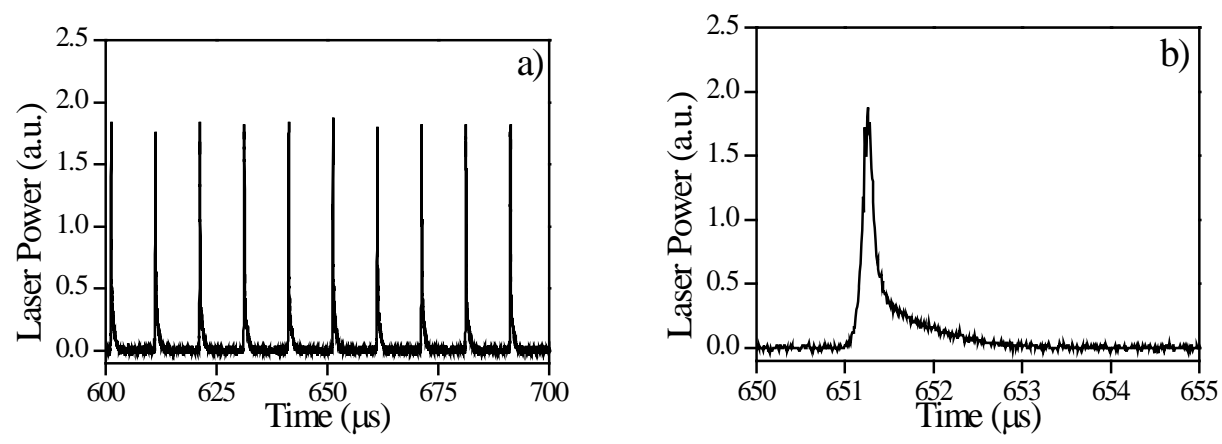

Fig. 4.2 a) Oscilloscope trace of the Q-switching pulses at $100 \mathrm{kHz}$ rate; and b) a single pulse with $82 \mu \mathrm{J}$ energy and $148 \mathrm{~ns}$ (FWHM) pulse width.

The pump power amplitude is set to $77 \mathrm{~W}$ and the pump time is adjusted to $2.2 \mu \mathrm{s}$ per cycle (i.e. 22\% duty cycle), to prevent the onset of the secondary pulse in each cycle. The short duration of pump time indicates that the laser had the potential to operate at a higher repetition rate, and thus, further increase its average output power. The generated pulse train is steady. When the laser is operated at $100 \mathrm{kHz}$, the peak amplitude variation was about 3\% standard deviation and the pulse 
duration variation of less than $1 \%$. At lower repetition rates, such as $10 \mathrm{kHz}$ and $60 \mathrm{kHz}$, stable pulses were also obtained, as shown in Fig. 4.3.
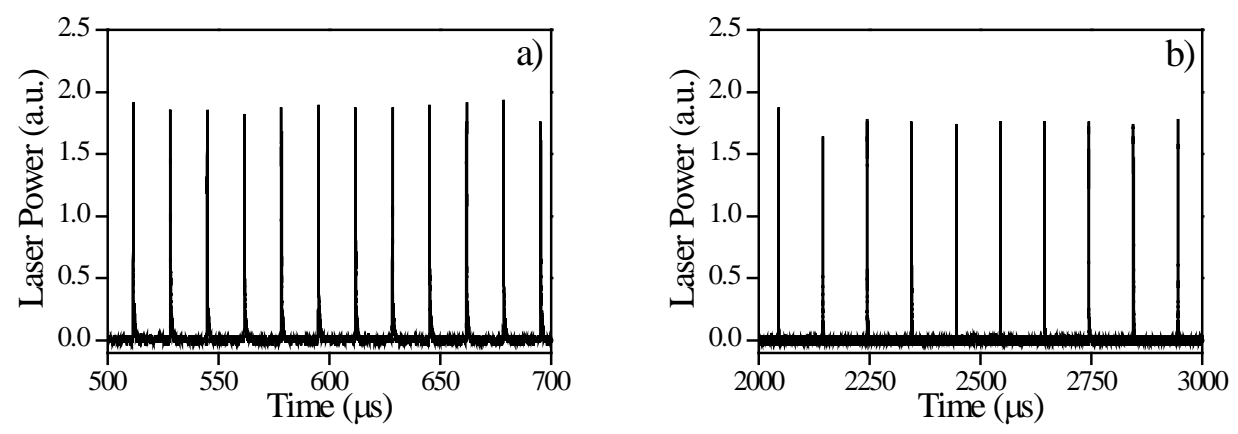

Fig. 4.3 Oscilloscope traces of the Q-switching pulses at a) $60 \mathrm{kHz}$, and b) $10 \mathrm{kHz}$.

We recorded output laser spectrums at different pump amplitudes, as shown in Fig 4.4. At high pump amplitude of $77 \mathrm{~W}$, a broad peak centered at $1118 \mathrm{~nm}$ appears in the spectrum. This $1118 \mathrm{~nm}$ peak, about $54 \mathrm{~nm}$ longer than the main peak at $1064 \mathrm{~nm}$, might be caused by stimulated Raman scattering (SRS). However, the energy at $1118 \mathrm{~nm}$ is negligible compared to the main laser peak at $1064 \mathrm{~nm}$, which is $60 \mathrm{~dB}$ higher (i.e. one millionth of the peak power at $1064 \mathrm{~nm}$ ).
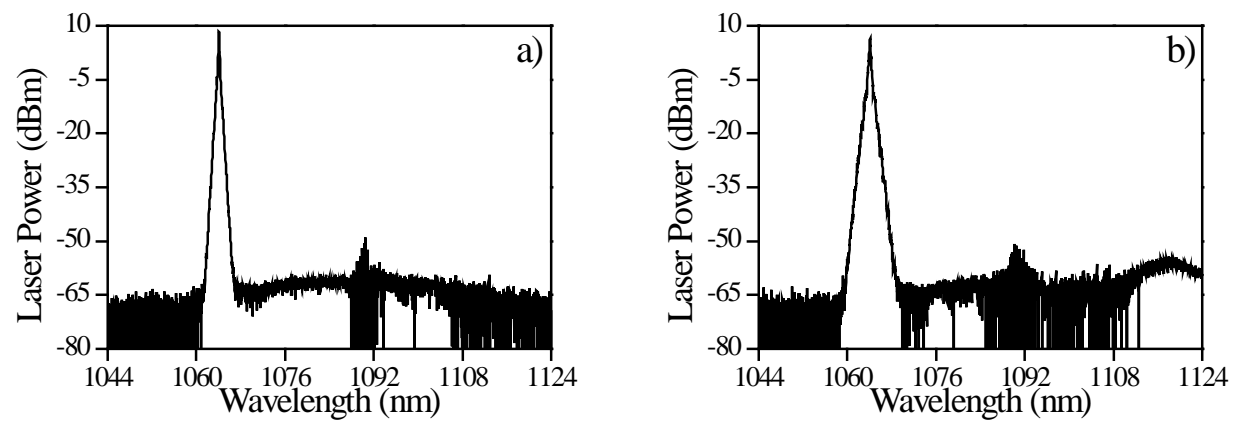

Fig. 4.4 Emission spectrum of the laser at a) $32 \mathrm{~W}$ of power amplitude (line width is 154 pm), and b) $77 \mathrm{~W}$ of power amplitude (line width is $230 \mathrm{pm}$ ).

In all recorded conditions, the laser output delivers a narrow spectral line width. The line width of the main spectral peak at $1064 \mathrm{~nm}$ increases with the pump power amplitude, as shown in Fig 4.5(a). The pump power amplitude increased from $32 \mathrm{~W}$ to $77 \mathrm{~W}$, and the spectral line width broadened from $154 \mathrm{pm}$ to 230 
$\mathrm{pm}$. Linear fitting is applied to the plot and the slope is estimated at $1.53 \mathrm{pm} / \mathrm{W}$. The steady slope indicates the potential to operate at high pump power amplitude and maintain relative narrow spectral line width.
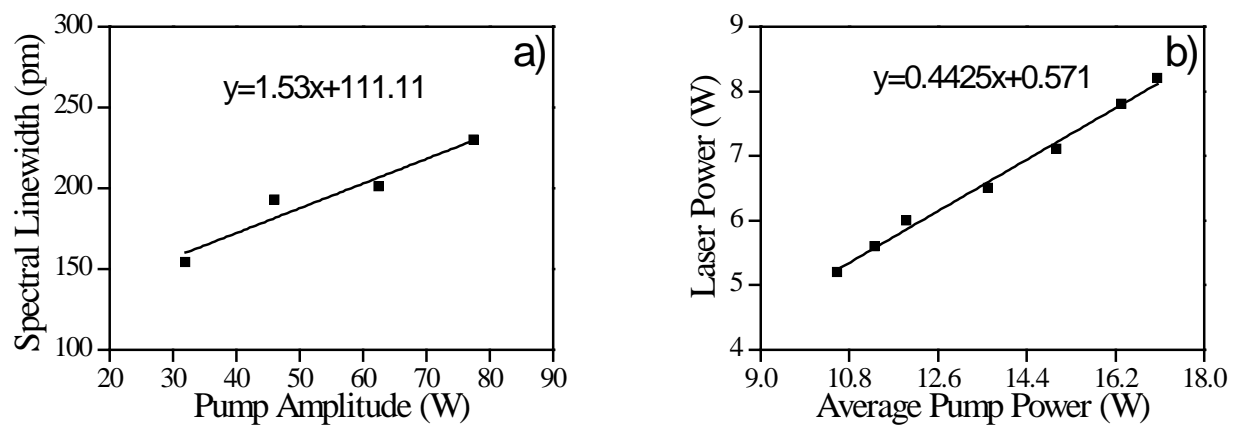

Fig. 4.5 a) Spectral line width versus pump power amplitude; and

b) Output laser power versus average pump power.

In Fig 4.5(b), the average pump power is calculated by multiplying pump amplitude with modulation duty cycle of the pump. Average output power is plotted against the average pump power to show the efficiency of the laser system. 8.2 W of output power is achieved while operated at $77 \mathrm{~W}$ of pump amplitude and 22\% duty cycle. Linear fitting is applied and the slope efficiency of $44 \%$ is achieved by the laser system. Such high efficiency is significant in a pulsed fiber laser, which is far exceeding the slope efficiency of 9\% in [15]. In addition, there is no trend of saturation, indicating the efficiency is well kept at higher pump power to generate higher peak power.

We measured the pump duration as function of pump power amplitude at pump modulation frequency of $100 \mathrm{kHz}$. The pump duration is fine adjusted for each pump amplitude to avoid the occurrence of the after pulses. In Fig. 4.6(a), as the pump amplitude increases from $32 \mathrm{~W}$ to $77 \mathrm{~W}$, the pump duration is reduced from $3.3 \mu \mathrm{s}$ to $2.2 \mu \mathrm{s}$. The corresponding laser pulse width and pulse energy were plotted in Fig. 4.6(b). The pulse width decreases from $200 \mathrm{~ns}$ to $148 \mathrm{~ns}$, and the pulse energy increases from $52 \mu \mathrm{J}$ to $82 \mu \mathrm{J}$. Higher pump amplitude leads to higher gain in the active fiber and thus takes less time depleting the population 
inversion after the on-set of the lasing. Therefore, higher pulse energy with a narrower pulse width is generated at higher pump amplitude. The pulse energy curve shows no saturation indicates high scalability for the pulse energy. However, the pulse width shows rolled over at around $70 \mathrm{~W}$ of pump amplitude. The factor that limiting the pulse width to go further short is under study.
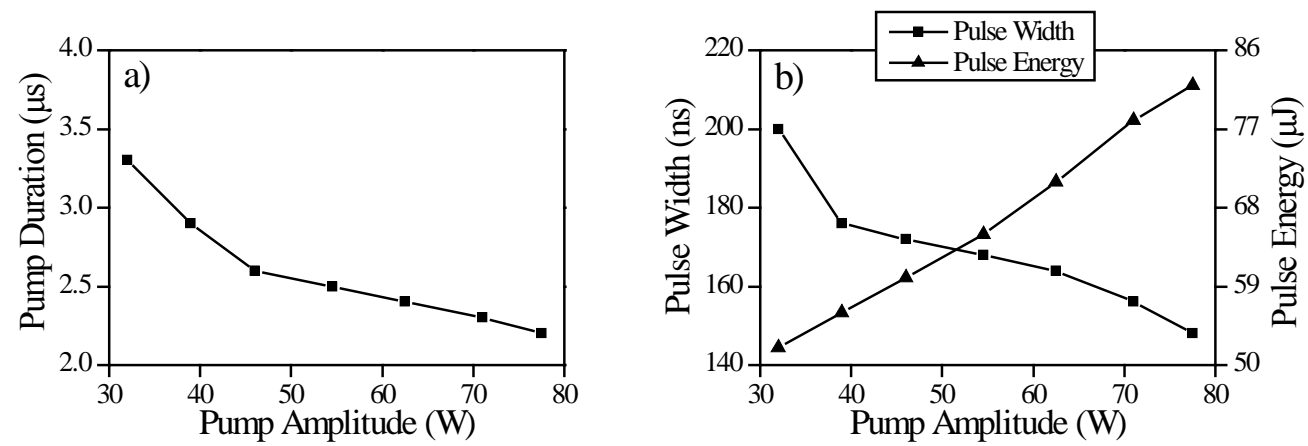

Fig. 4.6 a) Pump duration versus pump amplitude; and

b) laser pulse width and pulse energy versus pump power amplitude.

In order to further study the influence of each parameter and optimize the laser system, we conducted experiments as following: a) we fix the output grating to $9 \%$ reflection and change the active fiber length from $11 \mathrm{~m}$ to $8 \mathrm{~m}$; b) we fix the gain fiber length at $9 \mathrm{~m}$ and change the output grating reflection from $4 \%$ to $9 \%$. At each configuration, the laser is operated with $50 \mathrm{~W}$ of pump amplitude and 100 $\mathrm{kHz}$ of pump modulation frequency. The pump duration is adjusted freely to avoid the occurrence of after-pulses. The pulse width and pulse energy is measured and plotted against the changing parameters, as shown in Fig 4.7(a) and Fig 4.7(b). As the active fiber length increases from $8 \mathrm{~m}$ to $11 \mathrm{~m}$, the pulse width first decreases and then increases with local minimum of $170 \mathrm{~ns}$ at $9 \mathrm{~m}$. The pulse energy first increases and then decreases with local maximum of $66 \mu \mathrm{J}$ at $9 \mathrm{~m}$, as shown in Fig 4.7(a). Thus, $9 \mathrm{~m}$ of active fiber length is the local optimum. The effect of output grating reflection is shown in Fig 4.7(b). Pulse width is about the same at $168 \mathrm{~ns}$ from $4 \%$ to $6 \%$ of output grating reflection and increases to $170 \mathrm{~ns}$ at $9 \%$. Pulse energy maximizes to $69 \mu \mathrm{J}$ at $6 \%$ of output grating reflection. Thus, the highest peak power is achieved when output grating reflection is $6 \%$. 

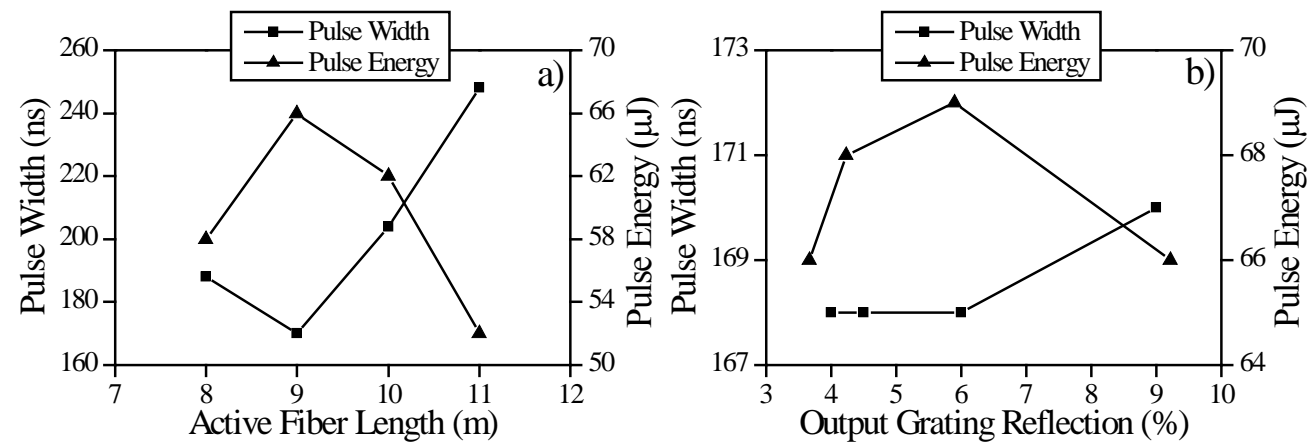

Fig. 4.7 Pulse width and pulse energy versus a) active fiber length, and b) output grating reflection, operated with pump power amplitude of $50 \mathrm{~W}$ at $100 \mathrm{kHz}$.

\subsection{Numerical model and optimization}

We use travelling wave method to develop the rate equations for the laser system. The rate equations governing the active fiber remain unchanged from chapter 2 . Because of the absence of SA fiber, the rate equations for SA fiber can be safely omitted. The boundary conditions become

$$
\begin{aligned}
& P_{p}^{+}(0, t)=P_{\text {pump }}(t) \\
& P_{k}^{-}(L, t)=(1-\eta) R_{O C} P_{k}^{+}(L, t) \\
& P_{k}^{+}(0, t)=(1-\eta) R_{H R} P_{k}^{-}(0, t)
\end{aligned}
$$

where $\eta$ is the average splice loss between active fiber and two gratings. Note that

the length of both gratings is neglected for simplicity. $R_{O C}$ and $R_{H R}$ are reflectivity of OC-FBG and HR-FBG, respectively. The non-linear effects, such as stimulated Brillouin scattering and stimulated Raman scattering, are negligible as well.

In this simulation, we use Yb-doped double cladding fiber from Nufern (LMAYDF-10/130-VIII) as active fiber. The cladding absorption coefficient is $3.9 \mathrm{~dB} / \mathrm{m}$ nominal at $975 \mathrm{~nm}$. The dopant concentration is estimated at $4.01 \times 10^{25} \mathrm{~m}^{-3}$. The spontaneous emission wavelength is considered spanning from $1030 \mathrm{~nm}$ to 
$1100 \mathrm{~nm}$. Other parameters used in the simulation are shown in Table 4.1 as given in Ref [18].

\begin{tabular}{|l|l|l|l|}
\hline Parameter & Value & Parameter & Value \\
\hline$N_{0}$ & $4.01 \times 10^{25} \mathrm{~m}^{-3}$ & $\tau$ & $0.84 \times 10^{-3} \mathrm{~s}$ \\
\hline$\sigma_{e k}(\mathrm{k}=1064 \mathrm{~nm})$ & $0.35 \times 10^{-24} \mathrm{~m}^{2}$ & $\sigma_{a k}(\mathrm{k}=1064 \mathrm{~nm})$ & $0.01 \times 10^{-24} \mathrm{~m}^{2}$ \\
\hline$\alpha_{k}($ for all $\mathrm{k})$ & $0.005 \mathrm{~m}^{-1}$ & $\alpha_{p}$ & $0.005 \mathrm{~m}^{-1}$ \\
\hline$\sigma_{a p}(\mathrm{p}=975 \mathrm{~nm})$ & $2.50 \times 10^{-24} \mathrm{~m}^{2}$ & $\sigma_{e p}(\mathrm{p}=975 \mathrm{~nm})$ & $2.44 \times 10^{-24} \mathrm{~m}^{2}$ \\
\hline$d_{\text {core }}$ & $10 \times 10^{-6} \mathrm{~m}$ & $d_{\text {cladding }}$ & $130 \times 10^{-6} \mathrm{~m}$ \\
\hline $\mathrm{R}_{\mathrm{OC}}$ & $9 \%$ & $\mathrm{R}_{\mathrm{HR}}$ & $99.9 \%$ \\
\hline NA & 0.075 & $\mathrm{~L}$ & $9 \mathrm{~m}$ \\
\hline
\end{tabular}

Table 4.1 Simulation parameters in passively Q-switched fiber laser with excessive long Yb-doped gain fiber.

The effect on of the pump on the far end of gain fiber is studied through the following simulations. In the proposed configuration, when the pump power is $70 \mathrm{~W}$, the residual pump power along the gain fiber is plotted in Fig 4.8(b). Position at 0 is where the pump power entering the fiber.
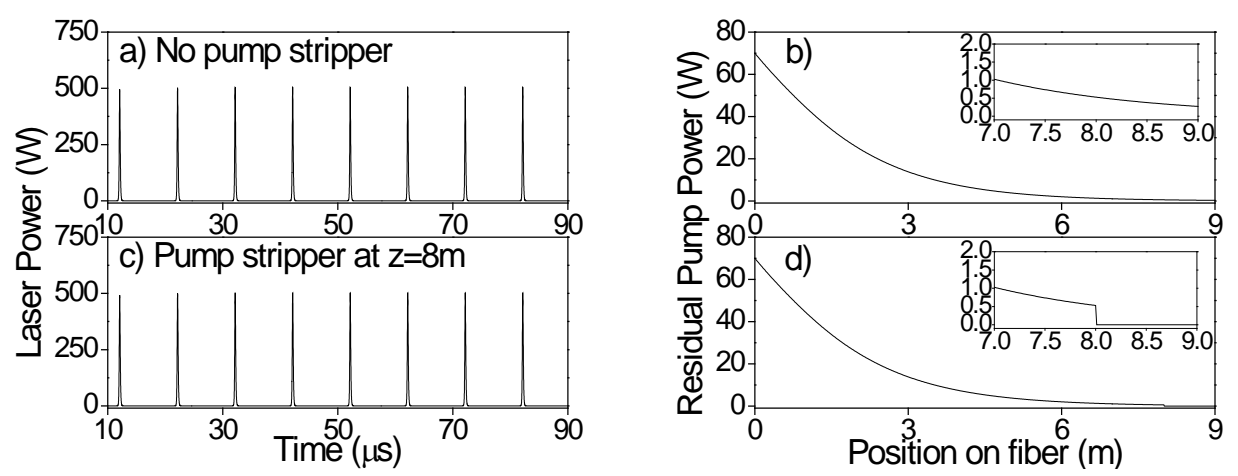

Fig. 4.8 a) Simulated output pulses for proposed configuration;

b) residual pump power along gain fiber for proposed configuration;

c) simulated output pulses with pump stripper at $8 \mathrm{~m}$; and

d) residual pump power along gain fiber with pump stripper at $8 \mathrm{~m}$.

We found out at the position of $8 \mathrm{~m}$, the residual pump power is less than $0.5 \mathrm{~W}$. Since the power confinement coefficient for this double cladding gain fiber is $0.6 \%$, the pump power can be neglected thereafter. The produced pulse train is 
plotted in Fig 4.8(a). To proof the residual pump power does not affect the output, we put a pump stripper at $8 \mathrm{~m}$ on the gain fiber. The resulting residual pump power is plotted in Fig 4.8(d), showing no pump power in the end $1 \mathrm{~m}$ of gain fiber. The produced pulse train is shown in Fig 4.8(c), which is almost identical to that shown in (a), the output from the proposed configuration. The difference of either pulse energy or pulse width is less than $0.3 \%$.

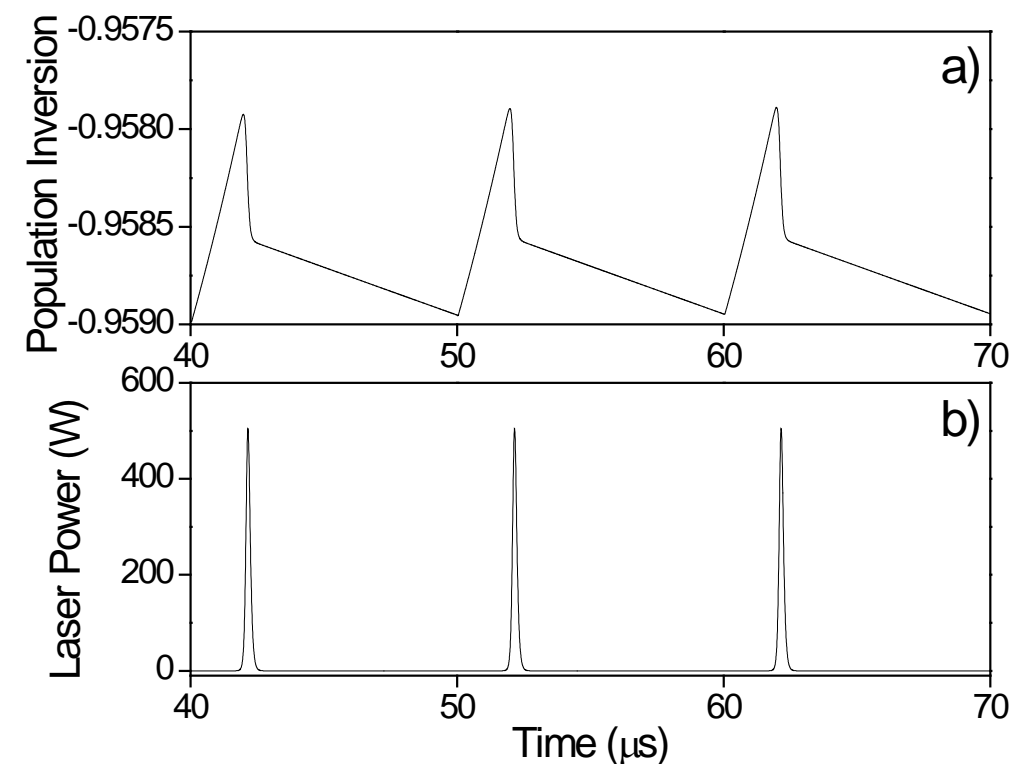

Fig. 4.9 a) Population inversion for the last $1 \mathrm{~m}$ of gain fiber;

b) corresponding simulated pulse train.

In the proposed configuration, the last $1 \mathrm{~m}$ of gain fiber is insufficiently pumped with only a small amount of active-ion excited into higher energy level. The evolution of population inversion for the last $1 \mathrm{~m}$ of gain fiber is shown in Fig 4.9(a). The corresponding pulse train is shown in Fig 4.9(b). Since this part of fiber is insufficiently pumped, the population inversion is mainly from absorption of ASE generated from pumped fiber. Therefore, for an excessive long gain fiber, the far end part of the fiber is hardly pumped and acting similar as SA, inducing the mechanism of Q-switching. 

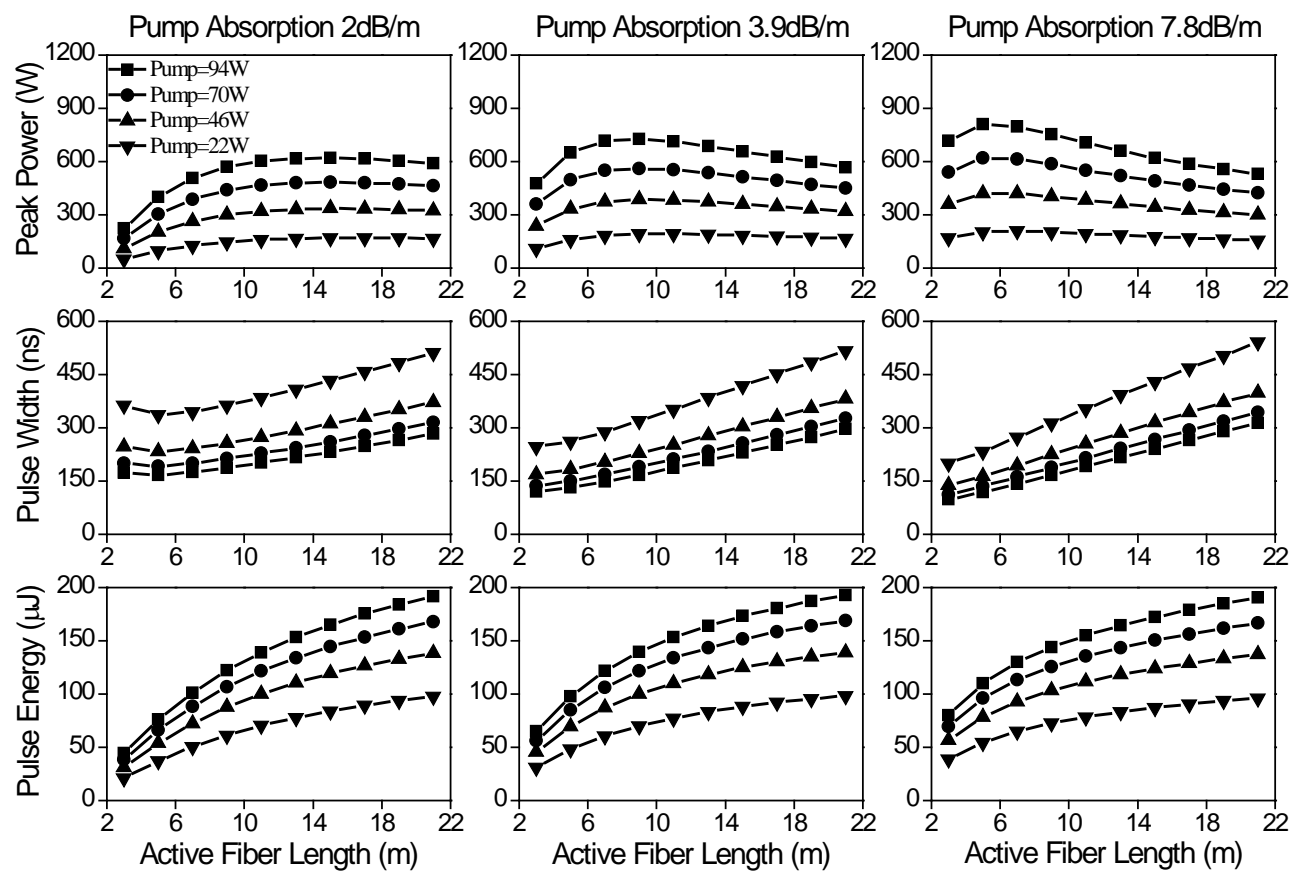

Fig. 4.10 Peak power, pulse width, and pulse energy of Yb doped fiber laser with different active fiber length, pump absorption ratio, and pump amplitude.

To study the effects of parameters and optimize the laser performance, we fix the pump modulation frequency at $100 \mathrm{kHz}$, and test under four pump power amplitude: $22 \mathrm{~W}, 46 \mathrm{~W}, 70 \mathrm{~W}$ and $94 \mathrm{~W}$. The pump duration is adjusted automatically to avoid the occurrence of after-pulses. The active fiber length and active fiber pump absorption ratio is studied first. While keeping the laser wavelength at $1064 \mathrm{~nm}$ and output coupler reflectivity at 9\%, the active fiber length changes from $3 \mathrm{~m}$ to $21 \mathrm{~m}$ for pump absorption ratio at $2 \mathrm{~dB} / \mathrm{m}, 3.9 \mathrm{~dB} / \mathrm{m}$ (used in the experiment), and $7.8 \mathrm{~dB} / \mathrm{m}$. The peak power, pulse width, and pulse energy is plotted as function of active fiber length in Fig 4.10. The peak power maximize to $620 \mathrm{~W}$ at $15 \mathrm{~m}$ with $2 \mathrm{~dB} / \mathrm{m}$ pump absorption, to $726 \mathrm{~W}$ at $9 \mathrm{~m}$ with $3.9 \mathrm{~dB} / \mathrm{m}$ pump absorption, and to $812 \mathrm{~W}$ at $5 \mathrm{~m}$ with $7.8 \mathrm{~dB} / \mathrm{m}$ pump absorption. For each pump absorption ratio, an optimum active fiber length exists. As the pump absorption ratio increases, the optimized peak power increases as well. The optimized active fiber length is shortened as pump absorption ratio increases, indicates the pump absorption ratio plays a scaling factor the fiber length. 
Although high pump absorption ratio has potential providing higher peak power, non-linear effect such as ion-pair clustering appears when the dopant concentration is too high, which leads to degrade the laser power [19, 20].
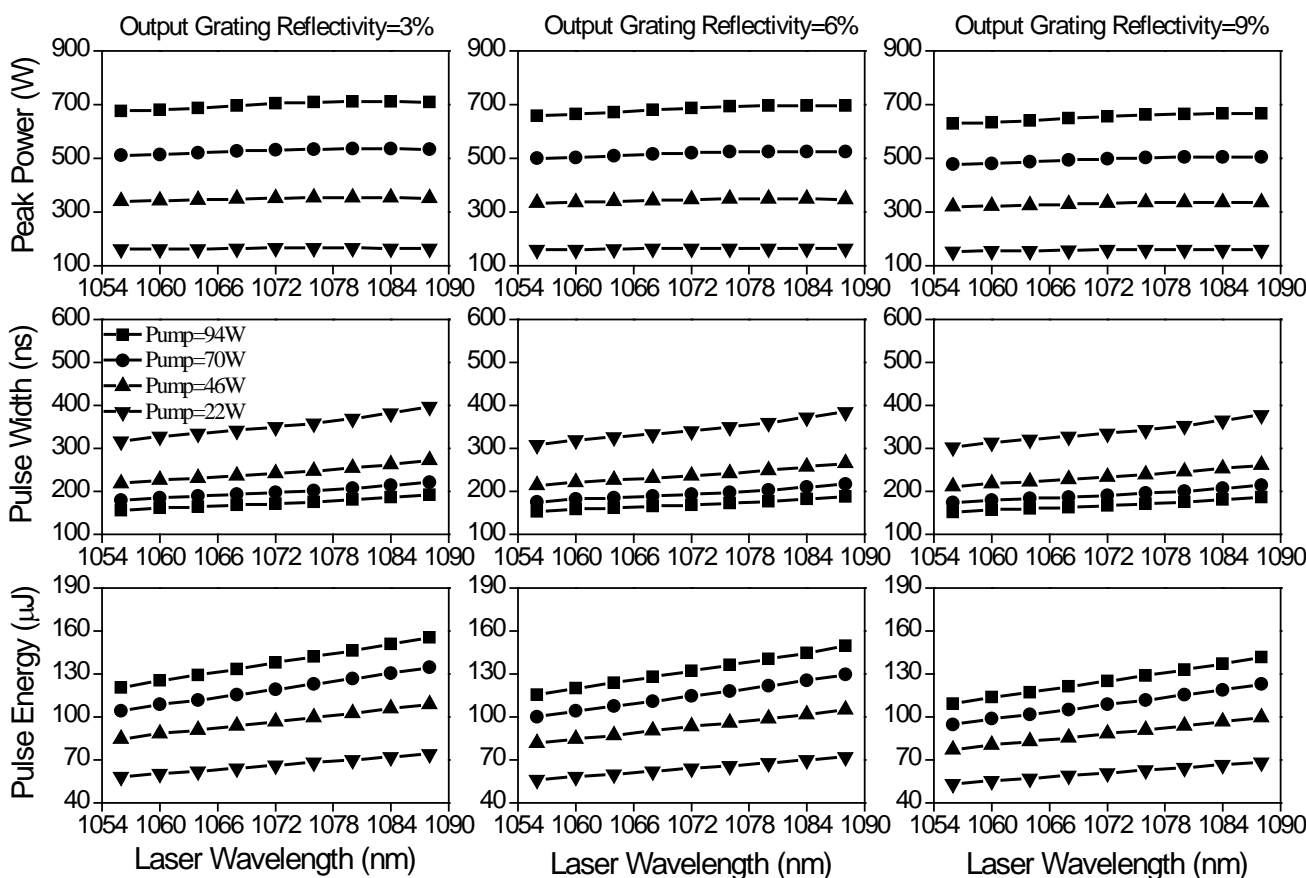

Laser Wavelength (nm)

Laser Wavelength (nm)

Fig. 4.11 Peak power, pulse width, and pulse energy of $\mathrm{Yb}$ doped fiber laser with different laser wavelength, output coupler reflectivity, and pump amplitude.

Other parameters such as output coupler reflectivity and lasing wavelength are also important for laser performance. For output coupler reflectivity at 3\%, 6\%, and $9 \%$, we set the active fiber length at $9 \mathrm{~m}$ with pump absorption at $3.9 \mathrm{~dB} / \mathrm{m}$, and change the laser wavelength from $1056 \mathrm{~nm}$ to $1088 \mathrm{~nm}$. The corresponding change of the peak power, pulse width, and pulse energy is shown in Fig 4.11. For a given output coupler reflectivity, the peak power merely changes with lasing wavelength. This gives designer freedom of choosing specific wavelength without sacrifice the output peak power. Also, the pulse energy increases as the lasing wavelength increases, thus, if higher pulse energy is preferred, increase the laser wavelength is an option. In addition, as output coupler reflectivity decreases, the pulse energy increases about $1.6 \%$ for every percentage of reflectivity decreases. 

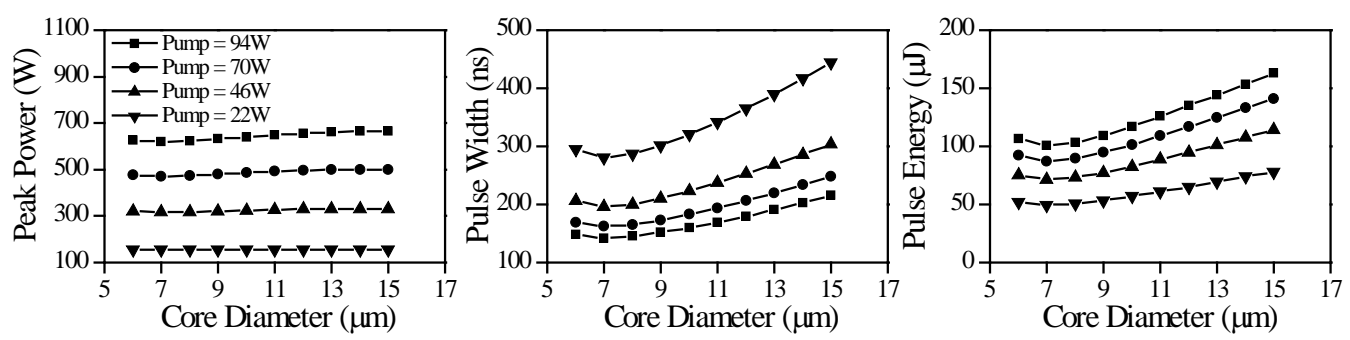

Fig. 4.12 Peak power, pulse width, and pulse energy of $\mathrm{Yb}$ doped fiber laser with different core diameters and pump amplitude.

Lastly, we study the laser performance as functions of active fiber core diameter. We set the active fiber length to $9 \mathrm{~m}$ with pump absorption at $3.9 \mathrm{~dB} / \mathrm{m}$ and change the active fiber core diameter from $6 \mu \mathrm{m}$ to $15 \mu \mathrm{m}$. Since we keep the cladding diameter constant at $130 \mu \mathrm{m}$, the dopant concentration is properly decreased to maintain the pump absorption at constant level. Peak power, pulse width, and pulse energy is plotted against core diameter as shown in Fig 4.12. The peak power increases about $6 \%$ from $6 \mu \mathrm{m}$ to $15 \mu \mathrm{m}$ core diameter when pump amplitude is $94 \mathrm{~W}$. Such an insignificant change is expected since the pump absorption ratio is kept constant. The pulse energy is minimized at $7 \mu \mathrm{m}$ and increases about $62 \%$ from $7 \mu \mathrm{m}$ to $15 \mu \mathrm{m}$. Thus, active fiber with larger core diameter produces higher pulse energy, which is preferred in many applications.

\subsection{Summary}

New configuration of passively Q-switched all-fiber laser has been proposed. The laser using an 9m long Yb-doped double cladding gain fiber without a heterogeneous SA fiber. The far end of the gain fiber is hardly pumped and thus acting like an SA to induce Q-switching. Simulation proves this assertion. The laser produces steady pulse train with $82 \mu \mathrm{J}, 148$ ns pulses which correspond to a peak power of $554 \mathrm{~W}$. The repetition rate of the laser is controllable by the repetition rate of the pump. At repetition rate of $100 \mathrm{kHz}$, slope efficiency of $44 \%$ is achieved. 
The laser output characteristics were investigated experimentally as functions of different parameters, such as pump amplitude, active fiber length, and output coupler reflectivity. The travelling wave model with wavelength dependency was used. In order to achieve higher pulse energy and peak power, in addition to increase the pump power, longer wavelength and larger core diameter are preferred. An optimal active fiber length could be determined by the developed theoretical model. 


\section{Chapter 5}

\section{Conclusion and future work}

\subsection{Conclusion of experiment}

In this thesis, three configurations of all-fiber passively Q-switched fiber laser have been presented. Designs using all-fiber configuration make the laser compact, reliable and maintenance free compare to the bulk design. By using saturable absorber fiber, the presented approaches produced stable and reliable higher peak power and pulse energy than those recently reported designs by other groups. Moreover, the repetition rate of the presented approaches is controlled by the frequency of the power source that drives the laser diodes, thus, enable a truly repetition rate controllable pulsed laser.

In the first configuration, a piece of Sm-doped fiber is used as SA. Mode field mismatch ratio of 3 has been obtained between $\mathrm{Yb}$-doped gain fiber and SA fiber. Steady pulse train with low timing jitter of less than $2 \%$ has been observed with $68 \mu \mathrm{J}, 210$ ns pulses.

Yb-doped fiber as SA fiber for passively Q-switched Yb-doped fiber laser has been presented in the second configuration. Secondary cavity with two strong gratings at $1100 \mathrm{~nm}$ is introduced in the design enclosing the SA fiber. This secondary cavity helps the excited ions in the SA quickly relax to the ground state after Q-switching. Thus, reduce the recovery time to return to the absorbing state. A factor of more than 100 times reduction is demonstrated through experiment. Mode field mismatch is also used in the configuration. Ratio of 4.6 is achieved by using large mode area (LMA) Yb-doped fiber of $15 \mu \mathrm{m}$ core. The laser produces pulses with short duration of $232 \mathrm{~ns}$ and high pulse energy of $86 \mu \mathrm{J}$. High stability of $1.8 \%$ timing jitter is also obtained. 
The third configuration assumes the insufficiently pumped part of the excessive long Yb-doped fiber acting similar to SA and has successfully obtained Qswitched pulses. Optimizations by changing the gain fiber length and reflectivity of output coupler have produced $82 \mu \mathrm{J} 148 \mathrm{~ns}$ pulses. Peak power is calculated to be $554 \mathrm{~W}$ without amplification stage. Also, the benefit of repetition rate being controlled by the modulation of pump finds applications in many industrial and research field.

\subsection{Conclusion of theoretical modeling}

Numerical modeling helps to understand the laser dynamics and optimization the laser design parameters in Q-switched lasers. Travelling wave models have been used to simulate the dynamics of passively Q-switched Yb-doped double clad fiber lasers. Numerical models have been modified for each configuration. All models are able to predict the temporal and spatial saturation of SA, which is critical to calculate the pulse characteristics.

The models have included the gain curves of the fiber for span from $1030 \mathrm{~nm}$ to $1100 \mathrm{~nm}$ and could qualitatively explain the dynamics of the behavior observed in the experiment. The laser performance versus several possible adjustable design parameters is studied through simulations. Laser output characteristics, including peak power, pulse energy, pulse duration, are determined by the evolutions of inversion distribution and ASE buildup, which depend on fiber length, dopant concentration, core diameter, output coupler reflectivity, wavelength, and most importantly pump amplitude. High dopant concentration in gain fiber is preferred as it is a scaling factor for short fiber length, and thus shortens pulse duration.

\subsection{Future work}

The peak power of the third approaches is limited by the threshold of SRS, which is depend mainly on the length and core size of the resonant cavity. Higher doping concentration and larger core size can be used to reduce the cavity length, thus 
increase the SRS threshold. The repetition rate of the proposed approaches has an upper limit. The period is calculated as the summation of the pump duration and the maximum restoration time required for the gain fiber and the SA fiber.

The all-fiber design, although has major advantage of compactness, robustness and freedom from adjustment, produces less peak power compare to its crystal SA counterpart. Yb-doped fiber with $\mathrm{Cr}^{4+}$ :YAG crystal SA produced $9 \mathrm{~kW}$ peak power in [12]. Improving laser performance by shortening pulse duration and increasing pulse energy is the first thing to do in the future.

Amplification stages could be added and optimized to greatly elevate the power delivered by the laser. Since for applications such as material processing, spectral line width is not essential [24], relative line width broadening as the result of amplification stage is acceptable. Comparison between providing more pump power for master oscillator or adding an amplification stage shall be experimentally tested.

The numerical model might be improved by including a heat-flow model. The emission and absorption cross sections of Yb-doped fiber are temperature dependent as it is a quasi-three level medium. Due to the unevenly distributed population inversion along fiber, the temperature profile in the fiber core area might change significantly within about 100ns to $1 \mu \mathrm{s}$ after a transient heating effect, which is comparable to the long tail time scale observed from Q-switched laser pulses [25 - 27]. More advanced model shall include the lateral cooling and temperature per each position along the fiber. 


\section{Appendix A}

\section{Calculation of Dopant Concentration}

\section{for Active Fibers}

This appendix summarizes the procedure used for calculating the dopant concentration for rare-earth doped active fiber from the absorption coefficient. For a commercially available active fiber, usually the absorption coefficient is given in the specification or more accurately in the data sheet. Otherwise, the absorption coefficient needs to be measured through experiment, which is not covered in this appendix. The absorption coefficient provided is usually with the unit of $\mathrm{dB} / \mathrm{m}$, denoted as $\alpha_{d B}$ so firstly, we need to convert it back to ratio as the formula given below:

$$
\alpha_{\text {eff }}=1-10^{-\left(\alpha_{d B} / 10\right)}
$$

Next step is to determine the power confinement factor $\Gamma_{k}$. For a double cladding fiber, $\Gamma_{k}=A_{\text {core }} / A_{\text {clad }}$, where $A_{\text {core }}$ and $A_{\text {clad }}$ are the area of core and clad, respectively. On the other hand, for single cladding, single mode active fiber, $\Gamma_{k}$ for the fundamental mode $\mathrm{LP}_{01}$ is usually equal to 0.82 [18]. Then the dopant concentration is calculated as below:

$$
N=\frac{\alpha_{e f f}}{\Gamma_{k} \cdot \sigma_{a k}}
$$

Where $\sigma_{a k}$ is the absorption coefficient for the given wavelength. 
For example, the gain fiber used in Chapter 2 and 3 is Nufern LMA-YDF-15/130VIII. The pump absorption rate is $5.4 \mathrm{~dB} / \mathrm{m}$ for $975 \mathrm{~nm}$, that is $\alpha_{d B}=5.4 \mathrm{~dB} / \mathrm{m}$. Then the effective absorption ratio is $\alpha_{\text {eff }}=0.7116 \mathrm{~m}^{-1}$ by using equation A.1. Since this is a double clad fiber, $\Gamma_{k}=A_{\text {core }} / A_{\text {clad }}=0.01331$. For Yb-doped silica fiber, $\sigma_{a k}=2.50 \times 10^{-24} \mathrm{~m}^{2}$ for $\lambda=975 \mathrm{~nm}$. Therefore, using equation A.2, the dopant concentration is $N=2.14 \times 10^{25} \mathrm{~m}^{-3}$.

These equations can also be used in the reverse order to determine the absorption ratio if given dopant concentration. 


\section{Appendix B}

\section{Material Processing Application}

The purpose of this appendix is to demonstrate the feasibility on material processing application, such as cutting and drilling, by using proposed laser system. In this experiment, the laser configuration proposed in Chapter 2 with the following modification is used. The gain fiber is replaced with 10/130 Yb-doped double cladding fiber and two $25 \mathrm{~W}$ diodes provided the total pump power of 50 $\mathrm{W}$. The laser provides $28 \mu \mathrm{J}$ pulse energy with estimated peak power of $140 \mathrm{~W}$. The average power of this laser is $2.8 \mathrm{~W}$. The laser output from patch cord is first collimated by Apollo L60 collimator with focus length of $60 \mathrm{~mm}$, and then the collimated beam is focused by a doublet lens with focus length of $35 \mathrm{~mm}$. The final spot size is estimated at $5 \mu \mathrm{m}$. Therefore, the power intensity is 713 $\mathrm{MW} / \mathrm{cm}^{2}$.

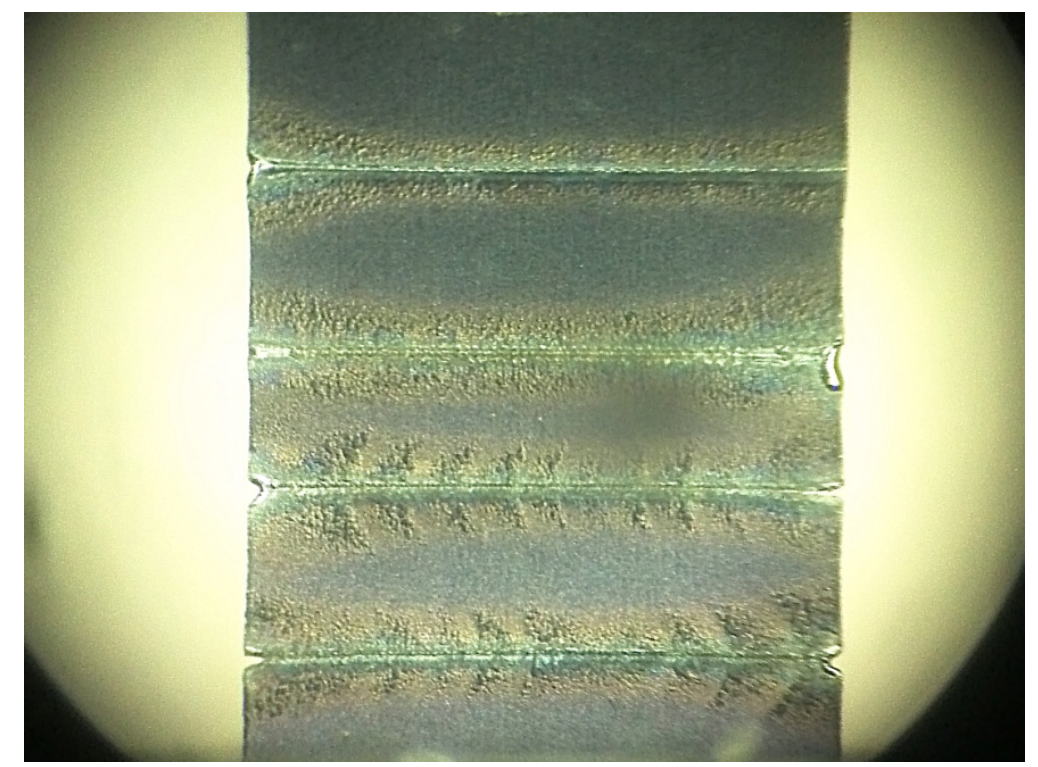

Fig. B.1 Titanium sheet metal of $136 \mu \mathrm{m}$ is cut at speed of $40 \mu \mathrm{m} / \mathrm{s}$ by the proposed fiber laser with $2.8 \mathrm{~W}$ average power, $100 \mathrm{kHz}$ repetition rate, $140 \mathrm{~W}$ peak power. 
The first specimen is a piece of titanium sheet metal with thickness of $136 \mu \mathrm{m}$. The specimen is fixed to a vertical stage which is placed at the focus point of the doublet lens. The movement of the stage is controlled by linear servo motors. The speed of motor can be adjusted and the maximum speed that allowing cutting through the metal is $40 \mu \mathrm{m} / \mathrm{s}$. Figure B.1 shows the cut trace on titanium sheet. The ability cutting through is provided by the peak power and the cutting speed is limited by the average power of the laser. Although the available speed is comparable low for the demonstrated experiment, since the laser performance is improved and the average power is increased 3 times, the cutting speed for the proposed laser in Chapter 4 might achieve $120 \mu \mathrm{m} / \mathrm{s}$ without any forms of amplification stage.

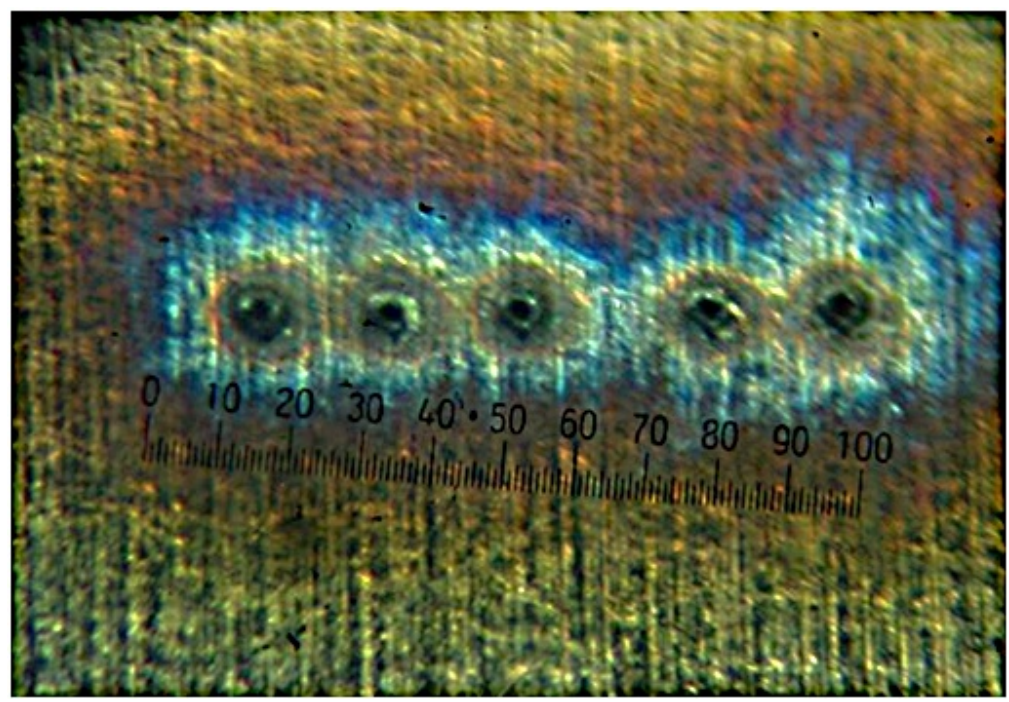

Fig. B.2 Stainless steel sheet metal of $151 \mu \mathrm{m}$ is drilled by the proposed fiber laser with $5.0 \mathrm{~W}$ average power, $100 \mathrm{kHz}$ repetition rate, $240 \mathrm{~W}$ peak power.

The second specimen is a piece of stainless steel sheet metal with thickness of 151 $\mu \mathrm{m}$. The laser used for drilling in this experiment is similar to the configuration in Chapter 4 with two $25 \mathrm{~W}$ pump diodes. The laser provides $240 \mathrm{~W}$ peak power and $5 \mathrm{~W}$ average power. The drilled hole is shown in Figure B.2.

The proposed fiber laser system shows promising application in material processing for its power, beam quality and compactness. 


\section{Appendix C}

\section{Abbreviations}

$\begin{array}{ll}\text { AOM } & \text { Acoustic optic modulator } \\ \text { APC } & \text { Angle polished connector } \\ \text { ASE } & \text { Amplified spontaneous emission } \\ \text { Bi } & \text { Bismuth } \\ \text { Cr } & \text { Chromium } \\ \text { CW } & \text { Continuous wave } \\ \text { DCF } & \text { Double cladding fiber } \\ \text { Er } & \text { Erbium } \\ \text { FBG } & \text { Fiber Bragg grating } \\ \text { FC } & \text { Fiber connector } \\ \text { FWHM } & \text { Full width at half maximum } \\ \text { HR } & \text { Highly reflective } \\ \text { LMA } & \text { Large-mode area } \\ \text { NA } & \text { Numerical aperture } \\ \text { OC } & \text { Output coupler } \\ \text { SA } & \text { Saturable absorber } \\ \text { SBS } & \text { Stimulated Brillouin scattering } \\ \text { SESAM } & \text { Semiconductor saturable mirror } \\ \text { Sm } & \text { Samarium } \\ \text { SRS } & \text { Stimulated Raman scattering } \\ \text { Tm } & \text { Thulium } \\ \text { YAG } & \text { Yttrium aluminum garnet } \\ \text { Yb } & \text { Ytterbium } \\ \text { YDF } & \text { Ytterbium-doped double cladding fiber }\end{array}$




\title{
Appendix D
}

\section{MATLAB Simulation Codes}

\author{
A1. Yb-Sm fiber laser \\ \%YbSm.m \\ function [t, Pout, Nd, Ndsa, Ppw] = YbSm(Laf, Naf, dcoreaf, Lsa, Nsa, dcoresa, laserk, Roc, \\ laserm, R1100, Pp, Pfreq, eta) \\ $\%$ Gain Medium: Yb doped Fiber \\ \%Saturable Absorber: Sm Fiber \\ $\%$ Active Fiber $(\mathrm{Yb})$ \\ dcladaf $=130 \mathrm{e}-6 ; \quad \%$ Inner cladding diameter of active fiber $(\mathrm{m})$ \\ naf $=1.45 ; \quad \%$ active fiber refractive index \\ NAaf $=0.075 ; \quad \%$ Numerical Aperture of active fiber \\ sigmaas $=1 \mathrm{e}-24 *[0.06190 .05490 .04830 .04260 .03780 .03340 .02920 .0250$ \\ 0.02100 .01750 .01450 .01200 .00990 .00880 .00840 .00770 .00670 .0056 \\ $0.00450 .00340 .00250 .00180 .00127 .3400 e-04$ 4.4100e-04 2.5100e-04 \\ 1.36ooe-04 6.9400e-05 3.3500e-05 1.5300e-05 6.5500e-06 2.6400e-06 \\ 9.9900e-07 3.5400e-07 1.1700e-07 3.6oooe-08]; \\ sigmaes $=1 \mathrm{e}-24 *[0.67300 .66600 .64900 .63300 .61400 .58600 .5520$ \\ 0.51700 .47600 .43500 .39500 .35700 .32100 .30000 .28400 .27800 .2720 \\ 0.26600 .25900 .25300 .24700 .24000 .23400 .22800 .22100 .21500 .2090 \\ $0.20200 .19600 .18900 .18300 .17600 .17000 .16300 .15700 .1500]$; \\ sigmaap $=2.5 \mathrm{e}-24 ; \quad \%$ absorption cross section of pump in active fiber ( $\mathrm{m}$ square) \\ sigmaep $=2.44 \mathrm{e}-24 i \quad \%$ emission cross section of pump in active fiber (m square) \\ alphap $=0.005 i \quad \%$ attenuation coefficient of active fiber at pump wavelength $(/ \mathrm{m})$ \\ alphas $=0.005 i \quad \%$ attenuation coefficient of active fiber at signal wavelength $(/ \mathrm{m})$ \\ tauaf $=0.84 \mathrm{e}-3 ; \quad \%$ fluorescence lifetime in active fiber (s) \\ Nafo $=$ Naf*2.14e25i \%doping concentration of active fiber (/m cubic) $5 \mathrm{~dB} / \mathrm{m}$ \\ gammap $=$ dcoreaf^ $2 /$ dcladaf $^{\wedge} 2 ; \%$ power filling factor of pump wavelength \\ $\%$ Saturable Absorber (Sm) \\ dcladsa $=125 \mathrm{e}-6 ; \quad \%$ cladding diameter of SA fiber $(\mathrm{m})$ \\ nsa $=1.45 i \quad \% S A$ fiber refractive index \\ NAsa $=0.14 i \quad \%$ Numerical Aperture of SA fiber \\ sigmaasa $=1 \mathrm{e}-24 *[0.05720 .06100 .06380 .06600 .06750 .06860 .06940 .0699$ \\ 0.07030 .07050 .07060 .07070 .07070 .07080 .07080 .07080 .07080 .0708 \\ 0.07080 .07080 .07080 .07080 .07080 .07080 .07080 .07080 .07070 .0707 \\ $0.07060 .07040 .07010 .06980 .06960 .06950 .06960 .0697] ;$ \\ sigmaesa $=0 ; \quad \%$ emission cross section of signal wavelength in SA (m square) \\ alphasa $=0.005 i \quad \%$ attenuation coefficient of SA fiber at signal wavelength $(/ \mathrm{m})$ \\ tausa $=5 \mathrm{e}-9 ; \quad$ \%upper state ion lifetime in SA fiber (s) \\ $\mathrm{Nsao}=\mathrm{Nsa}{ }_{1} .45 \mathrm{E}_{25} ; \quad \%$ doping concentration of SA fiber (/m cubic) \\ $\%$ Pump wavelength \\ lambdap = 975e-9; \%pump wavelength (m) \\ $\%$ Laser wavelength \\ lambdas $=1 e-9 *[10301032103410361038104010421044104610481050$
}




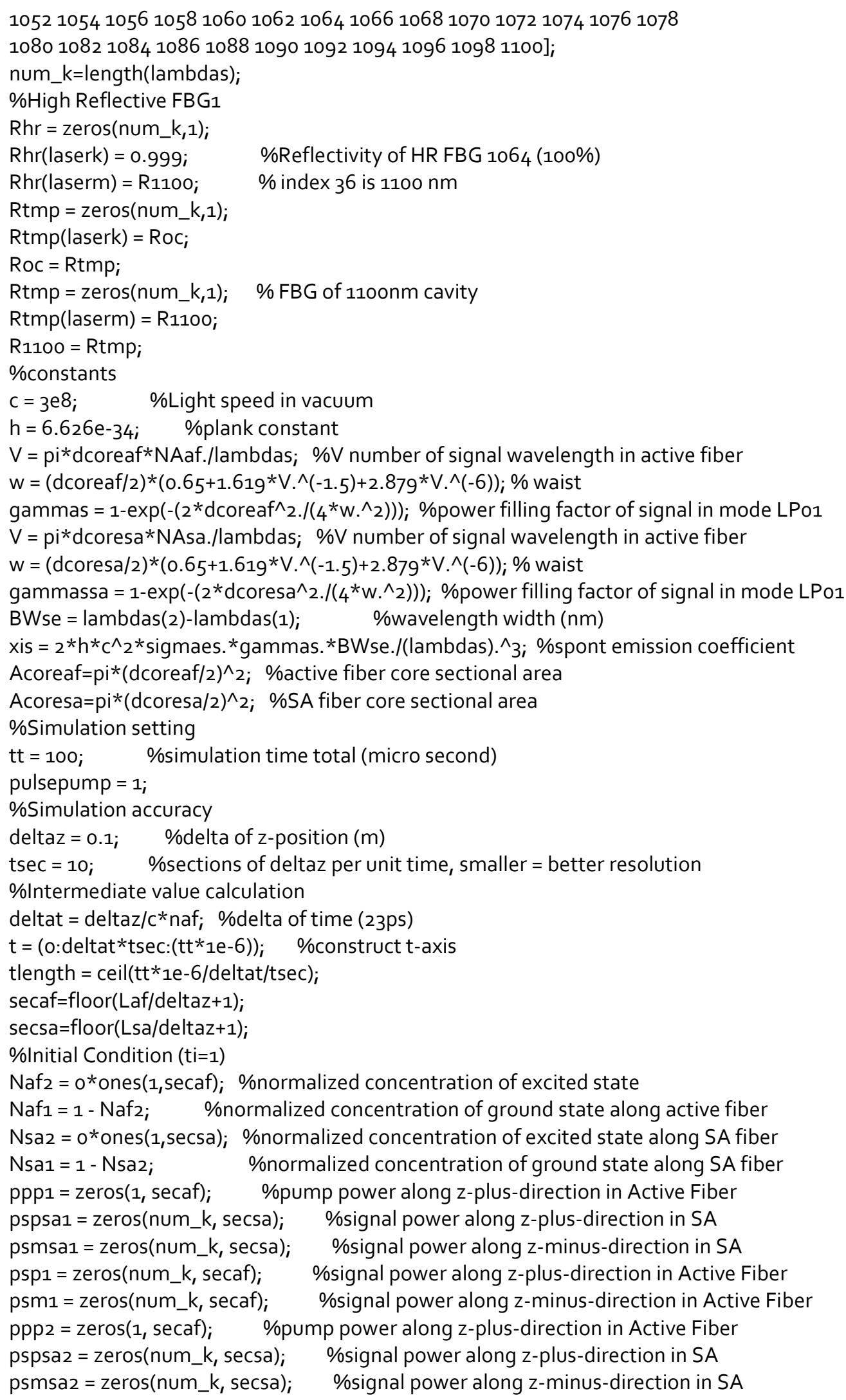




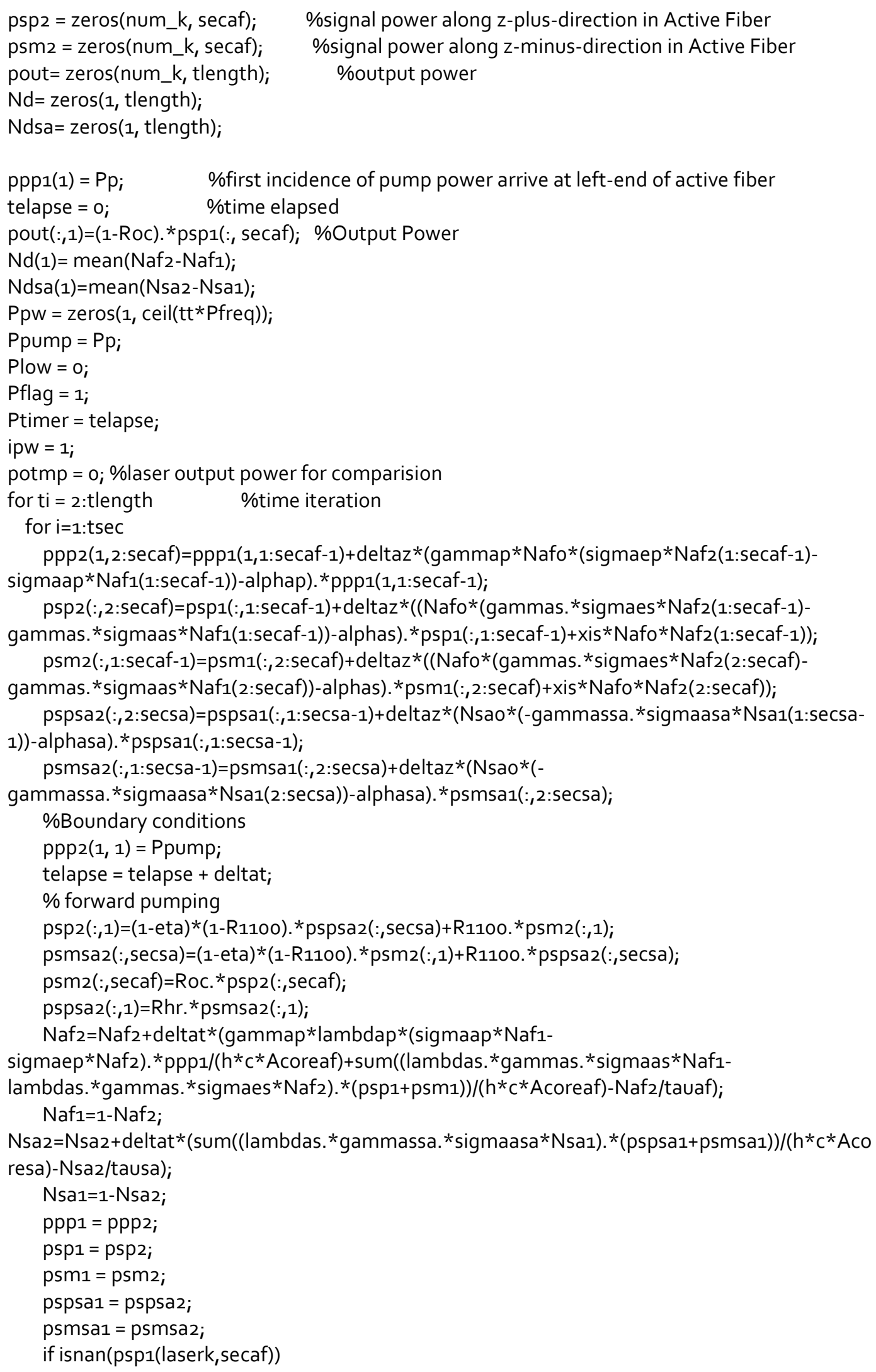




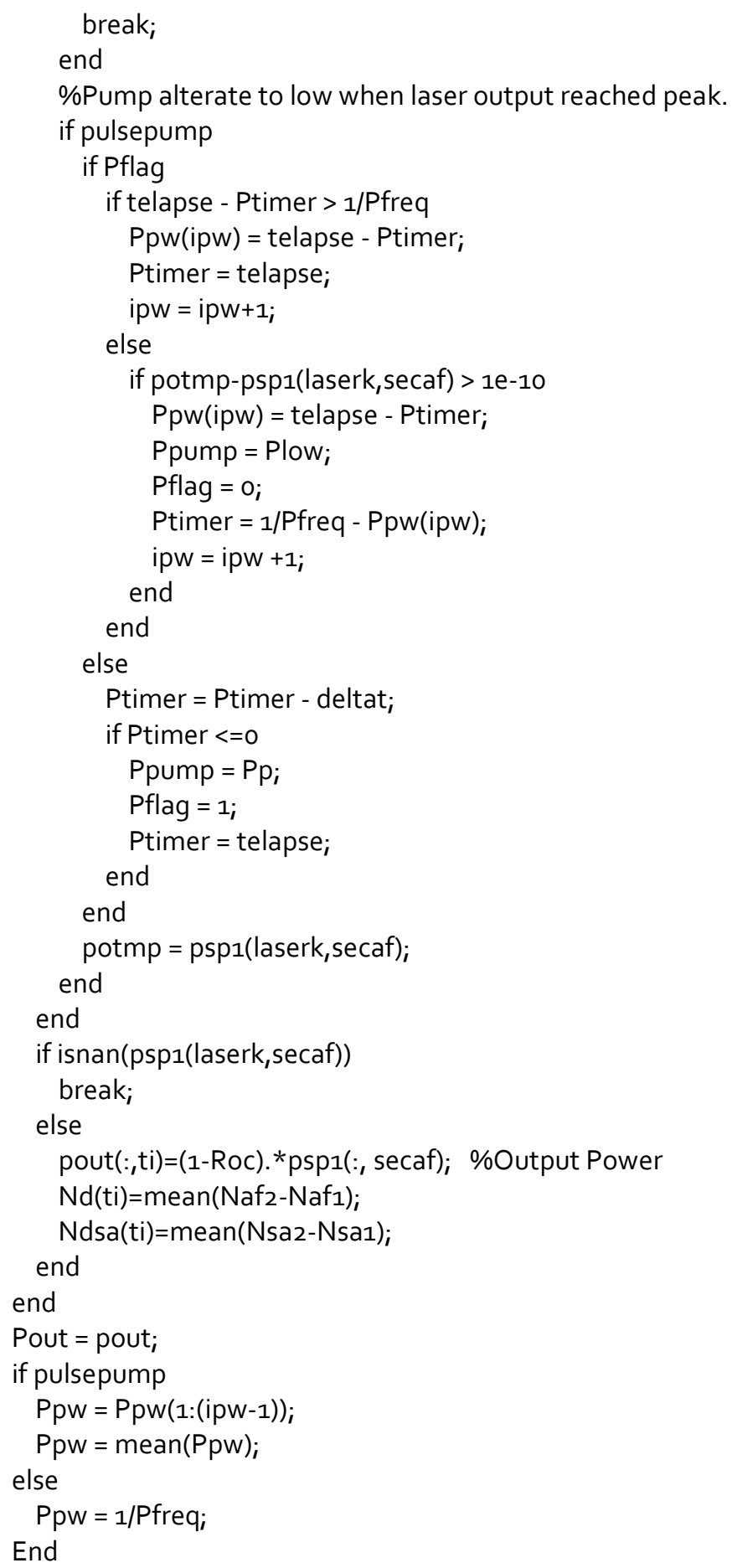

\section{A2. Yb-Yb fiber laser}

\%YbYbBack.m

function [t, Pout, Nd, Ndsa, Ppw] = YbYbBack(Laf, Naf, dcoreaf, Lsa, Nsa, dcoresa, laserk, Roc, laserm, R1100, Pp, Pfreq, eta) 
\%Gain Medium: Yb doped Fiber

\%Saturable Absorber: Yb Fiber

$\%$ Active Fiber (Yb)

dcladaf $=130 \mathrm{e}-6 ; \quad \%$ Inner cladding diameter of active fiber $(\mathrm{m})$

naf $=1.45 ; \quad \%$ active fiber refractive index

NAaf $=0.08 ; \quad \%$ Numerical Aperture of active fiber

sigmaas $=1 \mathrm{e}-24 *[0.06190 .05490 .04830 .04260 .03780 .03340 .02920 .02500 .0210$

0.01750 .01450 .01200 .00990 .00880 .00840 .00770 .00670 .00560 .00450 .0034

0.00250 .00180 .0012 7.3400e-04 4.4100e-04 2.5100e-04 1.36ooe-04 6.9400e-05

3.3500e-05 1.530oe-05 6.5500e-06 2.6400e-06 9.9900e-07 3.5400e-07 1.1700e-07

3.600oe-08];

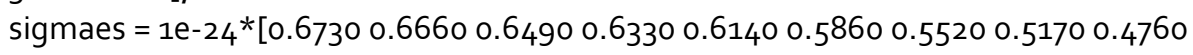

0.43500 .39500 .35700 .32100 .30000 .28400 .27800 .27200 .26600 .25900 .2530

0.24700 .24000 .23400 .22800 .22100 .21500 .20900 .20200 .19600 .18900 .1830

$0.17600 .17000 .16300 .15700 .1500] ;$

sigmaap $=2.5 \mathrm{e}-24 i \quad \%$ absorption cross section of pump in active fiber ( $m$ square)

sigmaep $=2.44 \mathrm{e}-24 i \quad \%$ emission cross section of pump in active fiber (m square)

alphap $=0.005 ; \quad \%$ attenuation coefficient of active fiber at pump wavelength $(/ \mathrm{m})$

alphas $=0.005 i \quad \%$ attenuation coefficient of active fiber at signal wavelength $(/ \mathrm{m})$

tauaf $=0.84 \mathrm{e}-3 ; \quad \%$ fluorescence lifetime in active fiber (s)

$\mathrm{Nafo}=\mathrm{Naf*} 2.14 \mathrm{e} 25 ; \quad \%$ doping concentration of active fiber (/m cubic)

gammap $=$ dcoreaf^ $2 /$ dcladaf $^{\wedge} 2 ; \%$ power filling factor of pump wavelength

\%Saturable Absorber (Yb)

dcladsa $=128 \mathrm{e}-6 ; \quad \%$ cladding diameter of SA fiber $(\mathrm{m})$

nsa $=1.45 i \quad \%$ SA fiber refractive index

NAsa $=0.19 ; \quad \%$ Numerical Aperture of SA fiber

sigmaasa $=1 \mathrm{e}-24 *[0.06190 .05490 .04830 .04260 .03780 .03340 .02920 .0250$

0.02100 .01750 .01450 .01200 .00990 .00880 .00840 .00770 .00670 .0056

$0.00450 .00340 .00250 .00180 .00127 .3400 e-04$ 4.4100e-04 2.5100e-04 1.3600e-04

6.9400e-05 3.3500e-05 1.5300e-05 6.5500e-06 2.6400e-06 9.9900e-07 3.5400e-07

1.1700e-07 3.6000e-08];

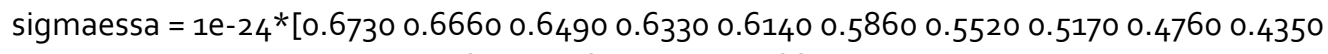

0.39500 .35700 .32100 .30000 .28400 .27800 .27200 .26600 .2590

0.25300 .24700 .24000 .23400 .22800 .22100 .21500 .20900 .20200 .19600 .1890

$0.18300 .17600 .17000 .16300 .15700 .1500]$;

alphasa $=0.005 i \quad \%$ attenuation coefficient of SA fiber at signal wavelength $(/ \mathrm{m})$

tausa $=0.84 \mathrm{e}-3 ; \quad \%$ upper state ion lifetime in SA fiber (s)

$\mathrm{Nsao}=\mathrm{Nsa}{ }^{*} 9.35 \mathrm{E} 25 ; \quad \%$ doping concentration of SA fiber (/m cubic)

\%Pump wavelength

lambdap $=975 \mathrm{e}-9 ; \quad \%$ pump wavelength $(\mathrm{m})$

\%Laser wavelength

lambdas = 1e-g*[103010321034103610381040104210441046104810501052

105410561058106010621064106610681070107210741076107810801082

10841086108810901092109410961098 1100];

num_k=length(lambdas);

\%High Reflective FBG1

Rhr = zeros(num_k,1);

Rhr(laserk) $=0.999 ; \quad$ \%Reflectivity of HR FBG 1064 (100\%)

Rtmp = Roc;

Roc $=$ zeros(num_k, 1 );

Roc(laserk) = Rtmp; 


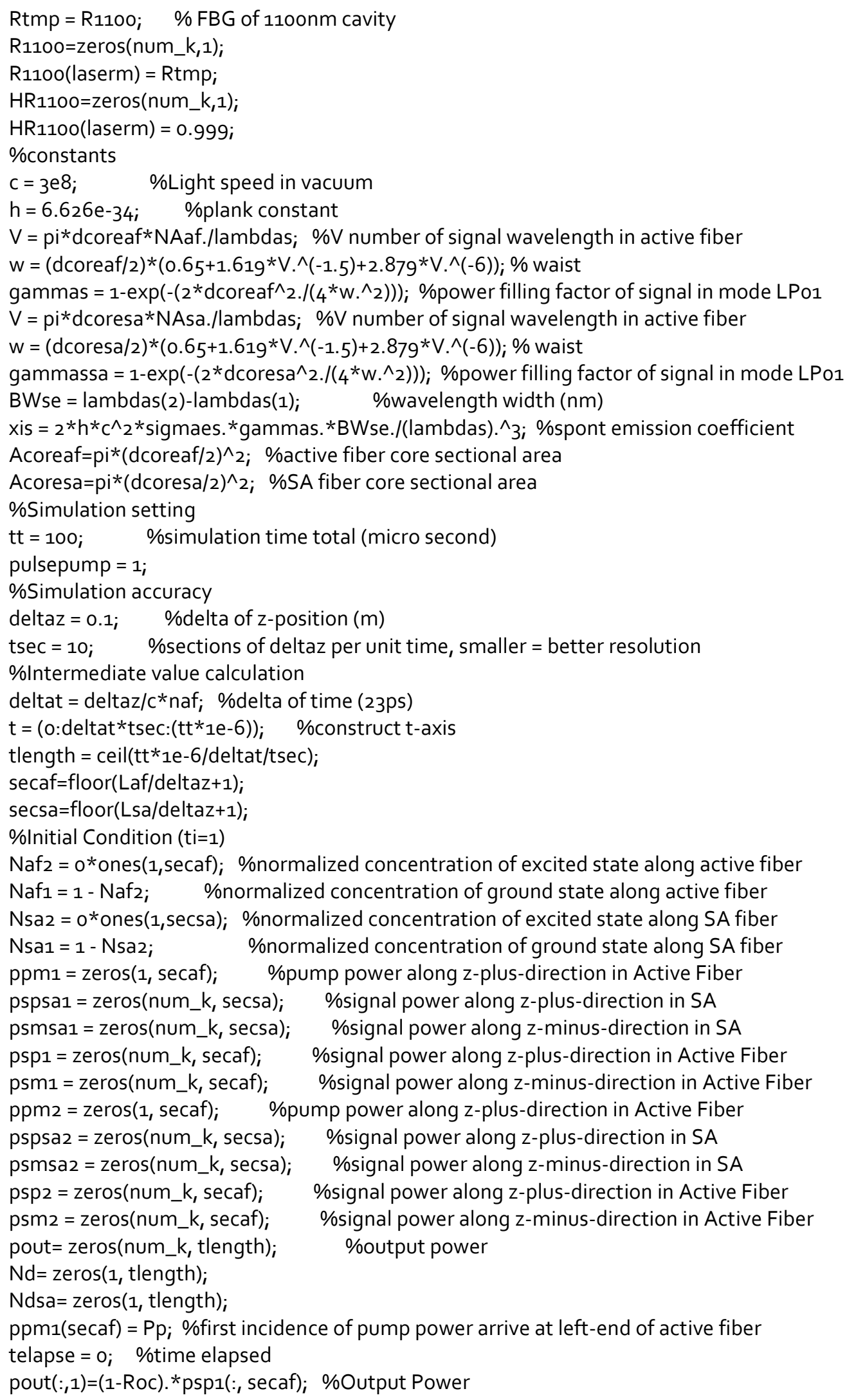




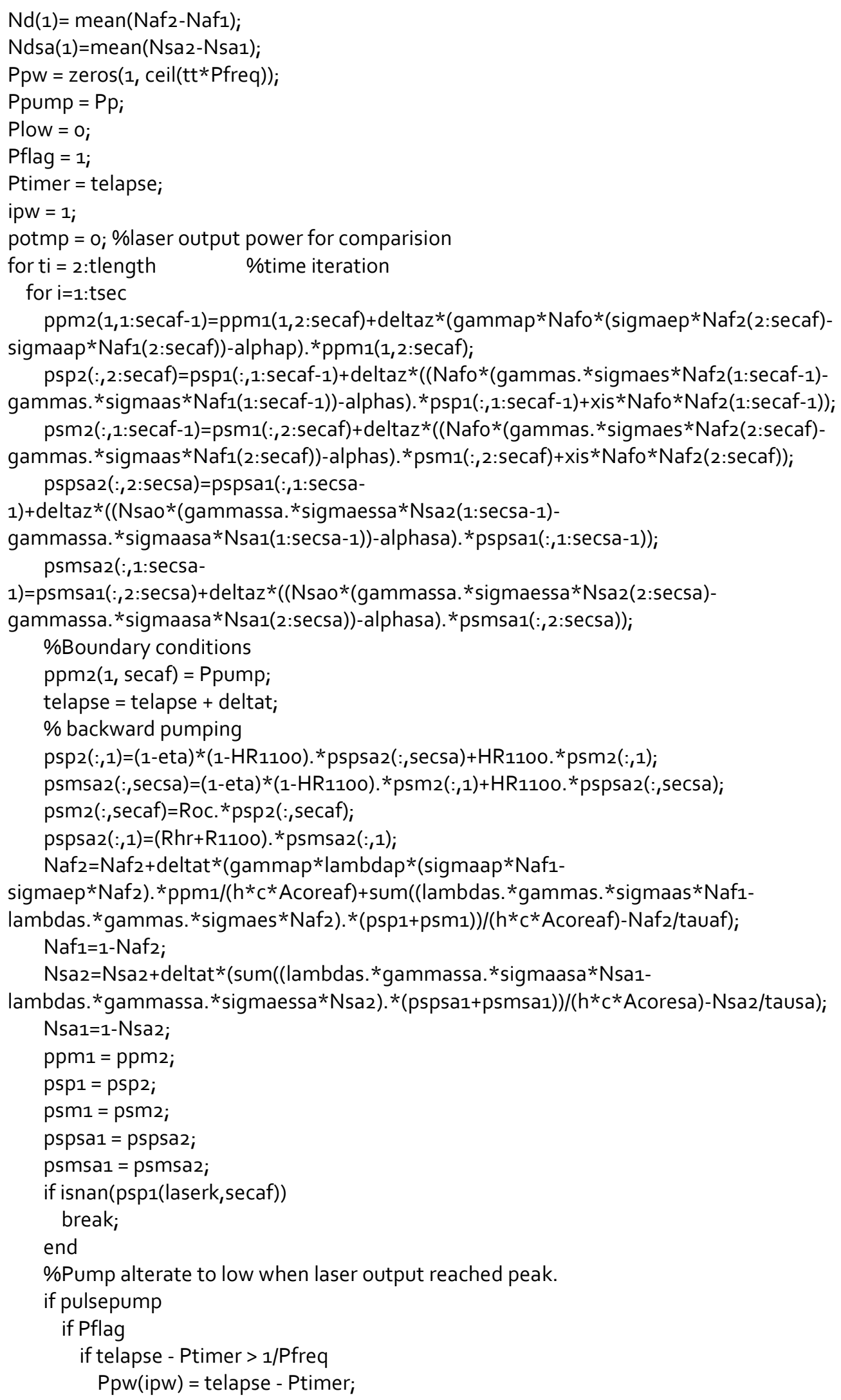




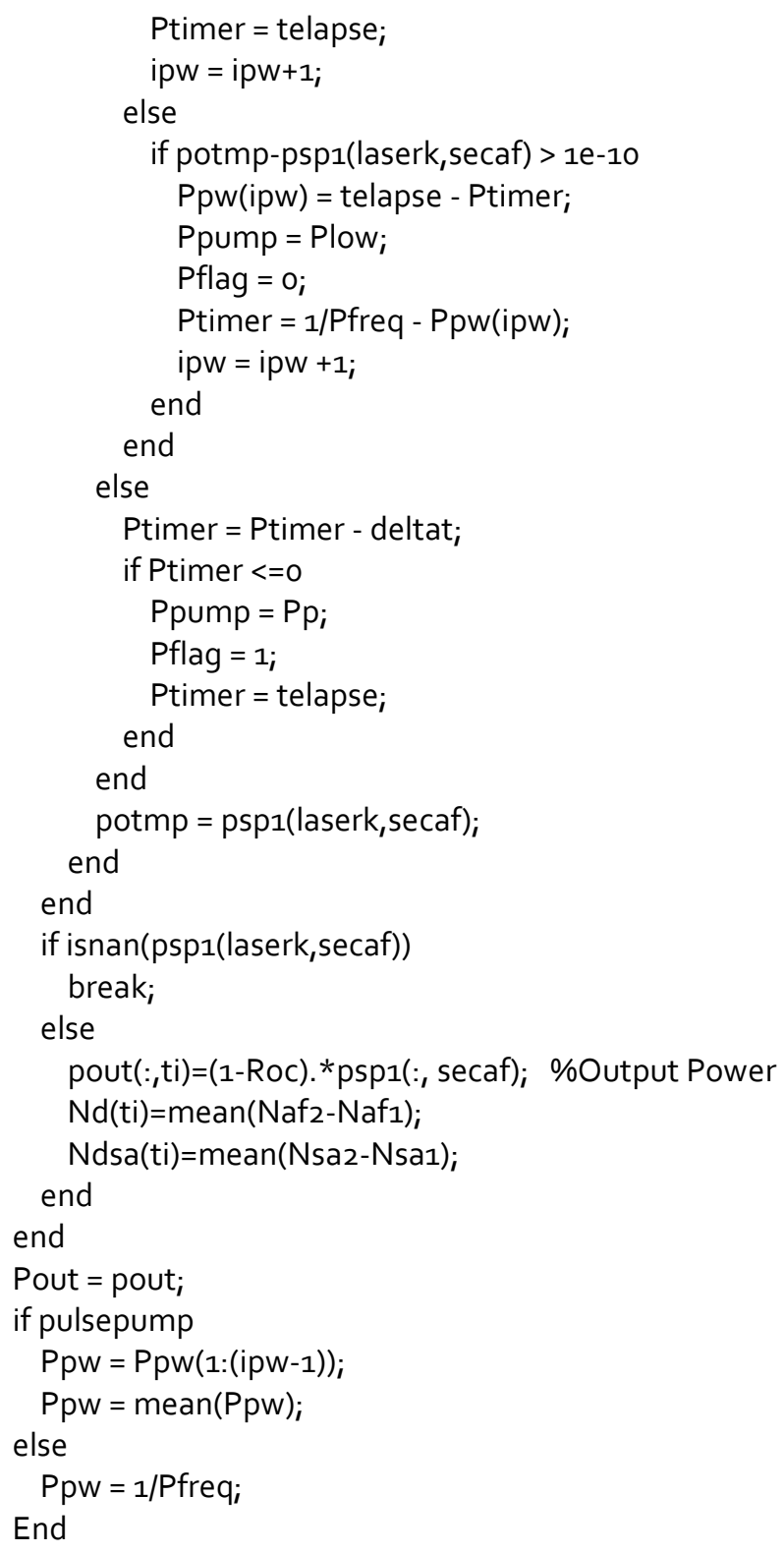

A3. Yb-only fiber laser

\%YbOnly.m

function [t, Pout, Nd, Ppw, ppp] = YbOnly(Laf, Naf, dcoreaf, laserk, Roc, laserm, R110o, Pp, Pfreq, eta) 


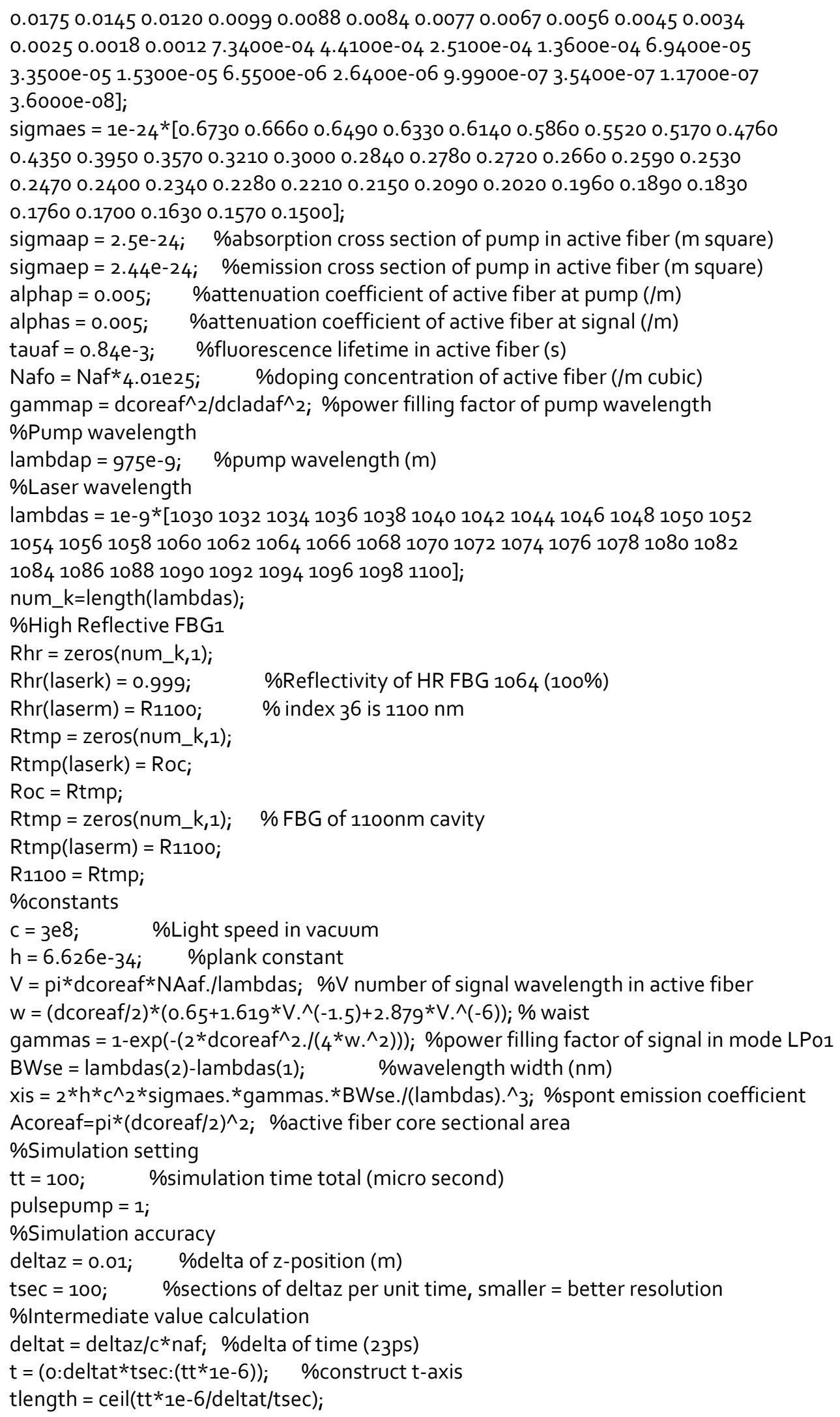




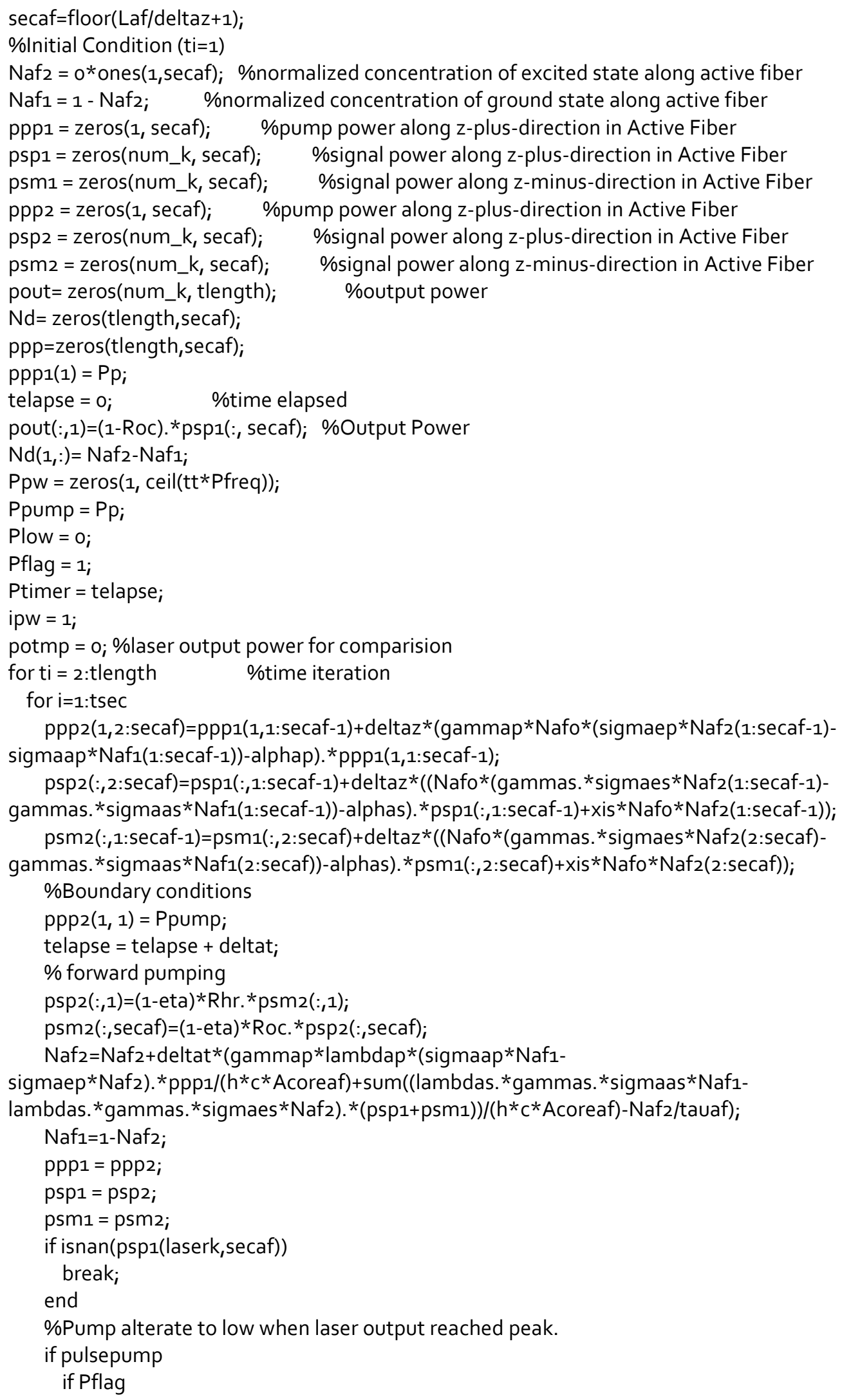




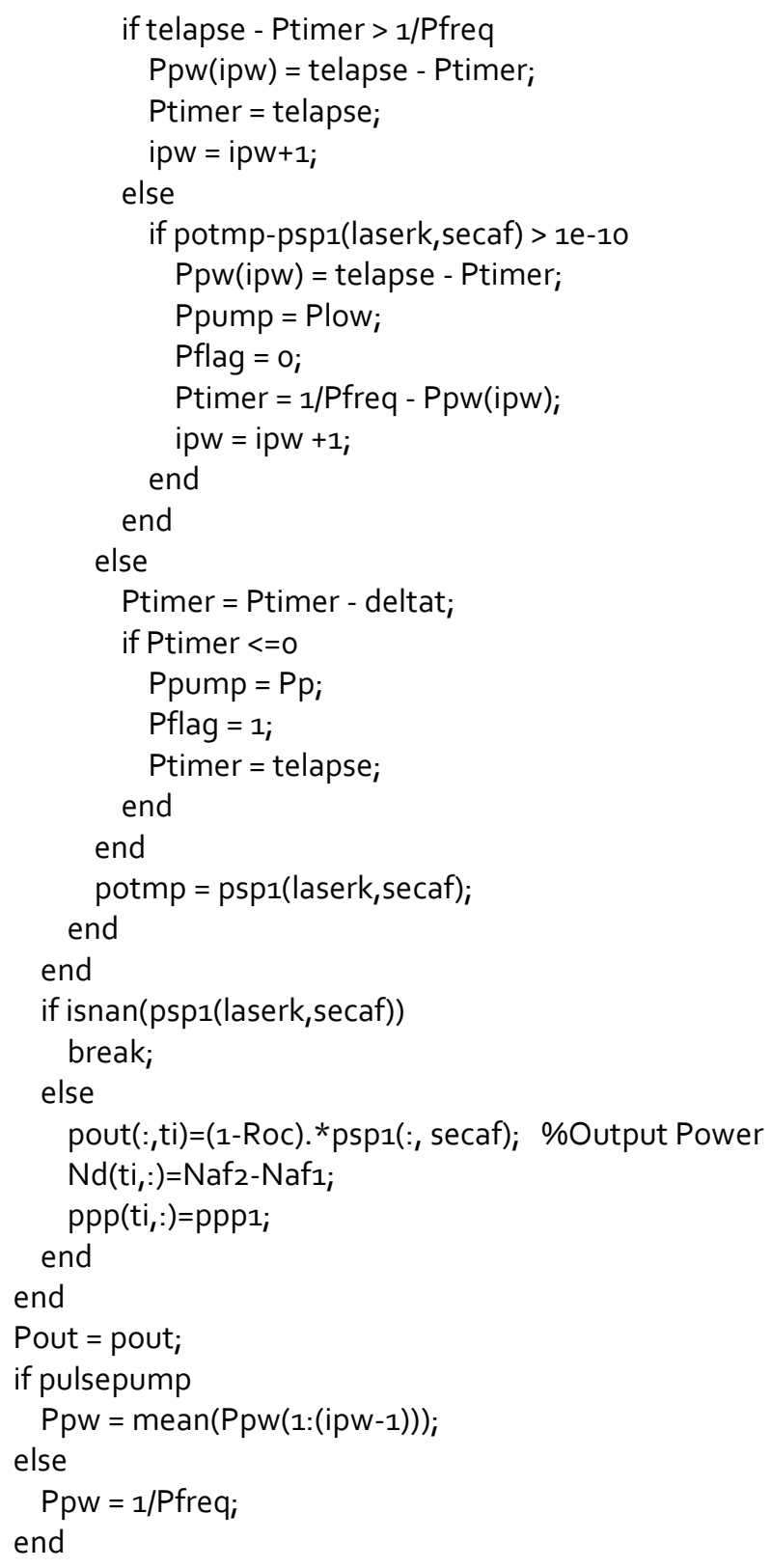




\section{References or Bibliography}

[1] Russo, N. A., et al. "High-efficiency Q-switched erbium fiber laser using a Bragg grating-based modulator." Optics communications 210.3 (2002): 361-366.

[2] Pérez-Millán, P., et al. "Q-switched all-fiber laser based on magnetostriction modulation of a Bragg grating." Optics Express 13.13 (2005): 5046-5051.

[3] Delgado-Pinar, M., et al. "Q-switching of an all-fiber laser by acoustooptic modulation of a fiber Bragg grating." Optics Express 14.3 (2006): 1106-1112.

[4] Kurkov, A. S. "Q-switched all-fiber lasers with saturable absorbers." Laser Physics Letters 8, no. 5 (2011): 335.

[5] Kurkov, A. S., et al. "All fiber Er-Tm Q-switched laser." Laser Physics Letters 7.11 (2010): 795-797.

[6] Wu, B., and P. L. Chu. "Fast optical switching in $\mathrm{Sm}^{3+}$-doped fibers." Photonics Technology Letters, IEEE 8.2 (1996): 230-232.

[7] Fotiadi, Andrei A., Andrei S. Kurkov, and I. M. Razdobreev. "All-fiber passively Q-switched Ytterbium laser." Lasers and Electro-Optics Europe, 2005. CLEO/Europe. 2005 Conference on. IEEE, 2005.

[8] A.A. Fotiadi, A.S. Kurkov, and I. Razdobreev, in: Proc. of the Conference on Lasers and Electro-Optics, Baltimore, MD, USA, May 611, 2007 (CLEO 2007), paper CMC4.

[9] A.A. Fotiadi, I. Razdobreev, B. Segard, and A. Kurkov, in: Proc. of the Conference on Lasers and Electro-Optics/Europe, Munich, Germany, June 14-19, 2009 (CLEO/Europe 2009), paper CJ3.5.

[10] Dvoyrin, Vladislav V., Valery M. Mashinsky, and E. M. Dianov. "Yb-Bi pulsed fiber lasers." Optics letters 32.5 (2007): 451-453.

[11] Tsai, Tzong-Yow, et al. "All-fiber passively Q-switched erbium laser using mismatch of mode field areas and a saturable-amplifier pump switch." Optics letters 34.19 (2009): 2891-2893.

[12] Laroche, M., et al. "Nanosecond pulse generation in a passively Qswitched Yb-doped fiber laser by $\mathrm{Cr}^{4+}$ : YAG saturable absorber." Photonics Technology Letters, IEEE 18.6 (2006): 764-766.

[13] Tsai, Tzong-Yow, et al. "Saturable absorber Q-and gain-switched all$\mathrm{Yb}^{3+}$ all-fiber laser at 976 and 1064 nm." Optics Express 18.23 (2010): 23523-23528. 
[14] Li, Zhi G., Zhengjun Xiong, Nicholas Moore, Gnian C. Lim, Wei L. Huang, and De X. Huang. "Amplified-spontaneous-emission effects in a passively Q-switched diode-pumped Nd: $\mathrm{YVO}_{4}$ laser." JOSA B 21, no. 8 (2004): 1479-1485.

[15] S. W. Moore, D. B. S. Soh, S.E. Bisson, B. D. Patterson, and W. L. Hsu, "400 $\mu \mathrm{J} 79$ ns amplified pulses from a Q-switched fiber laser using an Yb3+-doped fiber saturable absorber,” Opt. Express 20(21), 2 (2012): 3778-23789.

[16] Wang, Yong, and Chang-Qing Xu. "Modeling and optimization of Qswitched double-clad fiber lasers." Applied optics 45.9 (2006): 20582071.

[17] Thomas, James William. Numerical partial differential equations: finite difference methods. Vol. 1. Springer, 1995.

[18] L. Pan, "Experiment and Modeling of Passively Q-Switched Ytterbium Doped Double-Clad Fiber Lasers”, Oct 2010

[19] B. J. Ainslie , S. P. Craig-Ryan , S. T. Davey , J. R. Armitage , C. G. Atkins , J. F. Massicott and R. Wyatt "Erbium doped fibres for efficient optical amplifiers", Opt. Amplif. Commun. IEE Proc., vol. 137, pp.205 $-2081990$

[20] Blixt, P., J. Nilsson, T. Carlnas, and B. Jaskorzynska. "Concentrationdependent upconversion in $\mathrm{Er}^{3+}$-doped fiber amplifiers: Experiments and modeling." Photonics Technology Letters, IEEE 3, no. 11 (1991): 996-998.

[21] Kurkov, A. S., E. M. Sholokhov, and O. I. Medvedkov. "All fiber Yb Ho pulsed laser." Laser Physics Letters 6, no. 2 (2009): 135-138.

[22] D. B. S. Soh, S. E. Bisson, B. D. Patterson, and S. W. Moore, "Highpower all-fiber passively Q-switched laser using a doped fiber as a saturable absorber: numerical simulations,” Opt. Lett. 36(13), (2011): 2536-2538.

[23] J. Dawson, M. Messerly, R. Beach, M. Shverdin, E. Stappaerts, A. Sridharan, P. Pax, J. Heebner, C. Siders, and C. Barty, "Analysis of the scalability of diffraction-limited fiber lasers and amplifiers to high average power," Opt. Express 16, (2008): 13240-13266

[24] William M. Steen. Laser Material Processing. Springer, 2003.

[25] Ranaud, C. C., et al. "Characteristics of Q-switched cladding-pumped ytterbium-doped fiber lasers with different high-energy fiber designs." Quantum Electronics, IEEE Journal of 37.2 (2001): 199-206.

[26] Piper, A., et al. "High-power, high-brightness, mJ Q-switched ytterbiumdoped fibre laser." Electronics Letters 40.15 (2004): 928-929. 
[27] Huang, J. Y., et al. "Analytical model for optimizing the parameters of an external passive Q-switch in a fiber laser." Applied optics 47.13 (2008): 2297-2302.

[28] N. Jovanovic, "Novel Fibre Laser Sources based on Ultrafast Laser Inscribed Bragg Gratings”, Dec 2009

[29] Marcuse, D. "Pulsing behavior of a three-level laser with saturable absorber." Quantum Electronics, IEEE Journal of 29.8 (1993): 23902396.

[30] Luo, Liguo, and P. L. Chu. "Passive Q-switched erbium-doped fibre laser with saturable absorber." Optics communications 161.4 (1999): 257-263.

[31] Pask, H. M., et al. "Ytterbium-doped silica fiber lasers: versatile sources for the 1-1.2 $\mu \mathrm{m}$ region." Selected Topics in Quantum Electronics, IEEE Journal of 1.1 (1995): 2-13.

[32] Kurkov, A. S., E. M. Sholokhov, O. I. Medvedkov, V. V. Dvoyrin, Yu N. Pyrkov, V. B. Tsvetkov, A. V. Marakulin, and L. A. Minashina. "Holmium fiber laser based on the heavily doped active fiber." Laser Physics Letters 6, no. 9 (2009): 661-664.

[33] Kurkov, A. S., V. V. Dvoyrin, and A. V. Marakulin. "All-fiber $10 \mathrm{~W}$ holmium lasers pumped at $\lambda=1.15 \mu \mathrm{m}$." Optics letters 35 , no. 4 (2010): 490-492. 